\title{
Series Elastic Actuators
}

\author{
by \\ Matthew M. Williamson \\ M.Eng., University of Oxford (1993)
}

Submitted to the Department of Electrical Engineering and

Computer Science

in partial fulfillment of the requirements for the degree of

Master of Science

at the

\section{MASSACHUSETTS INSTITUTE OF TECHNOLOGY}

February 1995

(C) Massachusetts Institute of Technology 1995

Signature of Author .................................

Department of Electrical Engineering an Computer Science

January 20, 1995

Eng.

MASSACHIJSETTS INSTITUTE

- MUNOLOGY

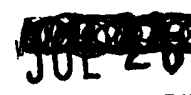

IDHARIES
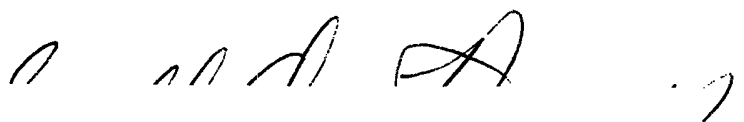

Certified by

Gill A. Pratt

Professor, Department of Electrical Engineering and Computer

Science

$\cap$ ก1, $\cap$ Thesis Supervisor

Accepted by

Chairman, Departmental Committee on Graduate Students 


\title{
Series Elastic Actuators \\ by \\ Matthew M. Williamson
}

Submitted to the Department of Electrical Engineering and Computer Science on January 20,1995, in partial fulfillment of the

requirements for the degree of

Master of Science

\begin{abstract}
This thesis presents the design, construction, control and evaluation of a novel force controlled actuator. Traditional force controlled actuators are designed from the premise that "Stiffer is better". This approach gives a high bandwidth system, prone to problems of contact instability, noise, and low power density. The actuator presented in this thesis is designed from the premise that "Stiffness isn't everything". The actuator, which incorporates a series elastic element, trades off achievable bandwidth for gains in stable, low noise force control, and protection against shock loads. This thesis reviews related work in robot force control, presents theoretical descriptions of the control and expected performance from a series elastic actuator, and describes the design of a test actuator constructed to gather performance data. Finally the performance of the system is evaluated by comparing the performance data to theoretical predictions.
\end{abstract}

Thesis Supervisor: Gill A. Pratt Title: Professor, Department of Electrical Engineering and Computer Science 


\section{Acknowledgments}

I would like to thank my advisor, Gill Pratt, for his continual drive to make me improve the actuator, and for his faith that it would always work better.

I would also like to thank those who have helped me over the year. I have appreciated discussions with, amongst others, Mike Binnard, Tim Tuttle, David Bailey, Rod Brooks, John Morrell, and Ackil Madhani.

Thank you to Cynthia Ferrell for proof reading parts of this thesis.

I would also like to thank Roz Gunby, Mark Cannon, Fiona Percy, Vladimir Alexeev and all the people in the loft for their love and support over the year.

This research was supported in part by the Jet Propulsion Laboratory, contract 959333 


\section{Contents}

1 Introduction 13

1.1 Approaches to Force Control . . . . . . . . . . . . . . . 13

1.2 Scope of Investigation . . . . . . . . . . . . . . 15

1.3 Review of Thesis Contents . . . . . . . . . . . . . . . . 15

$\begin{array}{lll}2 & \text { Literature Survey } & 17\end{array}$

3 Control and Modeling 21

3.1 Introduction . . . . . . . . . . . . . . . . 21

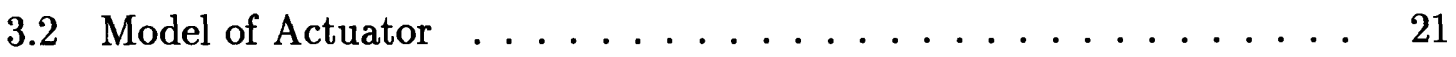

3.3 Feedforward Model . . . . . . . . . . . . . . . . . . 22

3.4 Feedback Control Scheme . . . . . . . . . . . . . . . . . 23

3.4 .1 Impedance and Stability . . . . . . . . . . . . . . . 23

3.4.2 Proportional, Integral and Derivative Control . . . . . . . 24

3.5 Proposed Control System . . . . . . . . . . . . . . . . 27

4 Performance Limits $\quad 29$

4.1 Introduction . . . . . . . . . . . . . . . . 29

4.2 Current Saturation . . . . . . . . . . . . . . . . . . 29

4.3 Comparison to a Stiff Actuator . . . . . . . . . . . 33

4.4 Velocity Saturation . . . . . . . . . . . . . . . . 35

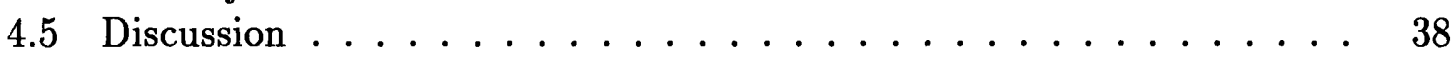

5 Actuator Design 39

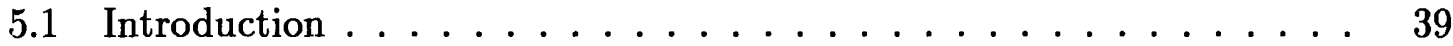

5.2 Choice of Motor . . . . . . . . . . . . . . . . . . . 40

5.3 Spring Design and Development . . . . . . . . . . . . . . 40

5.4 Actuator Spring Design . . . . . . . . . . . . . . . . . . . . . . 48

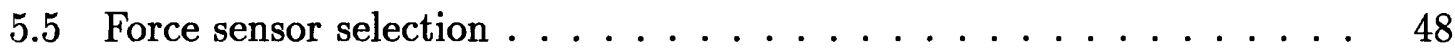

5.5 .1 Sensor 1 : Potentiometer . . . . . . . . . . . . 48

5.5 .2 Sensor 2 : Strain Gauges . . . . . . . . . . . . . . . . 49

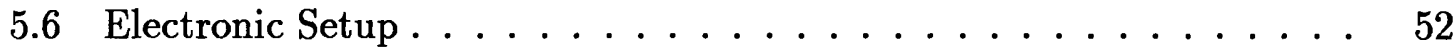

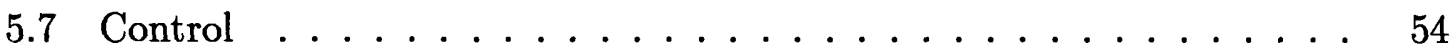


5.8 Test $\operatorname{Rig} \ldots \ldots \ldots \ldots \ldots \ldots \ldots \ldots \ldots \ldots$

6 Experimental Results $\quad 57$

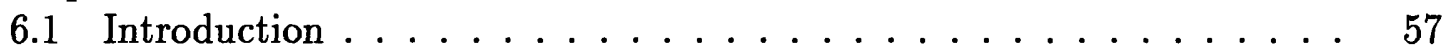

6.2 System Identification . . . . . . . . . . . . . . . . . . . 57

6.3 Feedback Tuning and Performance - Theoretical . . . . . . . . . 60

6.4 Feedback Tuning and Performance - Experimental . . . . . . . . . . 61

6.5 Assessing the effect of the $K_{f f}$ gain $\ldots \ldots \ldots \ldots \ldots \ldots$

6.6 System Performance . . . . . . . . . . . . . . . . 65

6.7 Performance Limits . . . . . . . . . . . . . . . . 67

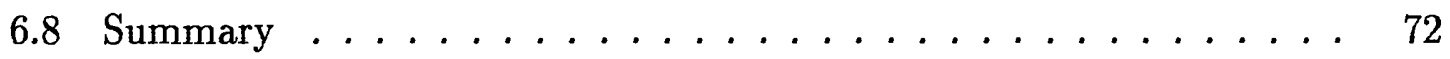

7 Applications $\quad \mathbf{7 3}$

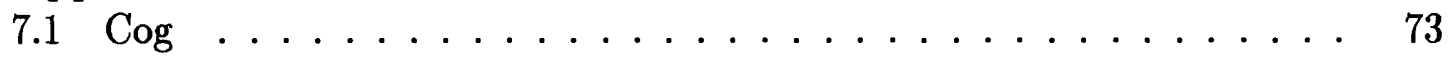

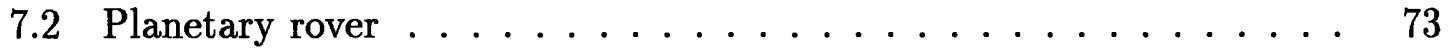

7.3 Biped Robot . . . . . . . . . . . . . . . . . 74

8 Conclusions $\quad 75$

8.1 Review of Thesis $\ldots \ldots \ldots \ldots \ldots \ldots \ldots \ldots \ldots$

8.2 Further Work . . . . . . . . . . . . . . . 75 


\section{List of Figures}

1-1 Schematic of series-elastic actuator $\ldots \ldots \ldots \ldots \ldots \ldots$

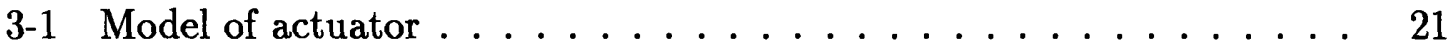

$3-2$ Plant model . . . . . . . . . . . . . . . . . . 23

3-3 Closed loop system . . . . . . . . . . . . . . . . . . 24

3-4 Plot of impedance for legal $\tau \ldots \ldots \ldots \ldots \ldots \ldots$

3-5 Proposed control system . . . . . . . . . . . . . 27

4-1 Phase diagram of motor forces . . . . . . . . . . . . . . 30

4-2 Maximum force v. impedance for below the natural frequency . . . . 31

4-3 Maximum force v. impedance at the natural frequency . . . . . . . 32

4-4 Maximum force v. impedance for above the natural frequency . . . . 32

4-5 Comparison of stiff and elastic actuators at low frequency . . . . . . 33

4-6 Comparison of stiff and elastic actuators at the natural frequency . . 34

4-7 Comparison of stiff and elastic actuators at high frequency . . . . . 34

$4-8$ Motor model . . . . . . . . . . . . . . . . . 35

4-9 Velocity saturation below the natural frequency . . . . . . . . 36

4-10 Velocity saturation at the natural frequency . . . . . . . . . 37

4-11 Velocity saturation above the natural frequency . . . . . . . . . 37

4-12 Motor movement for a stiff and an elastic actuator . . . . . . . . . 38

$5-1 \quad$ Photograph of actuator . . . . . . . . . . . . . . . 39

5-2 Schematic of mechanical design . . . . . . . . . . . 41

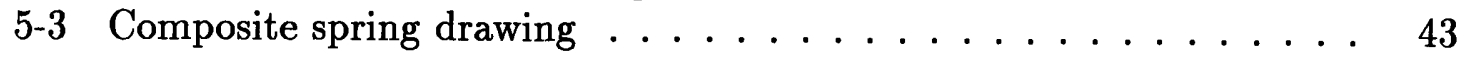

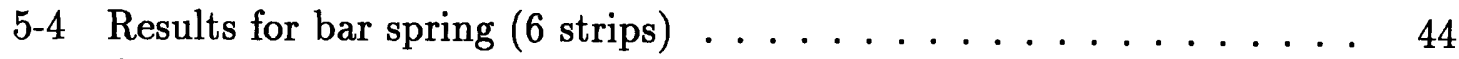

$5-5$ Cross Spring Drawing . . . . . . . . . . . . . . . . . 44

5-6 Cross spring photograph $\ldots \ldots \ldots \ldots \ldots \ldots \ldots$

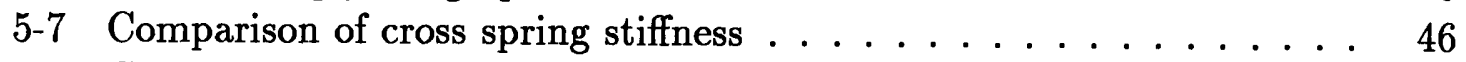

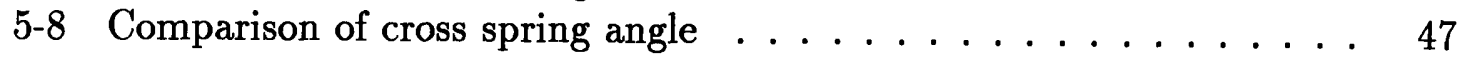

5-9 Comparison of cross spring torque . . . . . . . . . . . . 47

5 -10 Plot of spring torque against spring angle . . . . . . . . 48

5 -11 Schematic of potentiometer attachment . . . . . . . . . 49

$5-12$ Showing position of gauges on the spring $\ldots \ldots \ldots \ldots$

$5-13$ Closeup of spring . . . . . . . . . . . . . 50 
5-14 Plot of strain gauge reading versus potentiometer reading . . . . . . 51

5 -15 Plot of strain reading versus measured torque . . . . . . . . . 52

$5-16$ The complete system block diagram . . . . . . . . . . . . 52

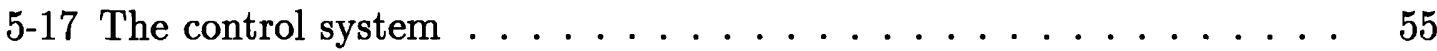

$5-18$ Motor model . . . . . . . . . . . . . . . 56

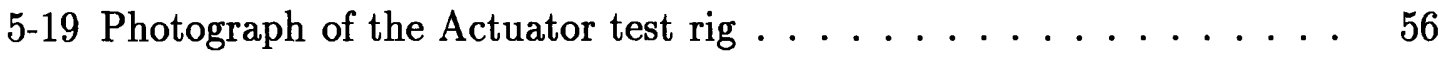

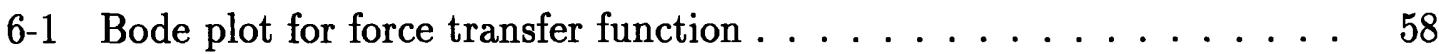

6-2 Bode plot for impedance transfer function . . . . . . . . . . . 58

6-3 The closed loop system . . . . . . . . . . . . . . . . . . 60

6-4 Bode plot for simulated closed loop force transfer function $\ldots . . .61$

6-5 Bode plot for simulated closed loop impedance transfer function . . . 62

6-6 Bode plot for identified closed loop force transfer function . . . . . . . 63

6-7 Bode plot of the dependence of the system closed loop response of backlash . . . . . . . . . . . . . . . . 63

6-8 Bode plot for identified closed loop impedance transfer function . . . 64

6-9 Bode plot showing effect of $K_{f f} \ldots \ldots \ldots \ldots \ldots \ldots \ldots$

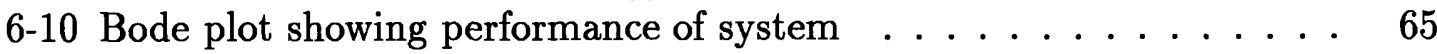

6 -11 Square wave response . . . . . . . . . . . . . 66

6 -12 Sine wave response . . . . . . . . . . . . . 66

6-13 Torque control performance when the output shaft is moving . . . . 67

6-14 Performance results for $12 \mathrm{rad} / \mathrm{sec} \ldots \ldots \ldots \ldots \ldots 68$

6-15 Performance results for $25 \mathrm{rad} / \mathrm{sec} \ldots \ldots \ldots \ldots \ldots \ldots$

6 6-16 Performance results for $37.7 \mathrm{rad} / \mathrm{sec} \ldots \ldots \ldots \ldots \ldots \ldots$

6-17 Performance results for $44 \mathrm{rad} / \mathrm{sec} \ldots \ldots \ldots \ldots \ldots \ldots . \ldots . \ldots 69$

6-18 Performance results for $44 \mathrm{rad} / \mathrm{sec}$, including efficiency $\ldots \ldots \ldots 70$

6-19 Performance results for $50.3 \mathrm{rad} / \mathrm{sec} \ldots \ldots \ldots \ldots \ldots \ldots$

6-20 Performance results for $62.8 \mathrm{rad} / \mathrm{sec} \ldots \ldots \ldots \ldots \ldots \ldots \ldots \ldots \ldots$

6-21 Performance results for $94.3 \mathrm{rad} / \mathrm{sec} \ldots \ldots \ldots \ldots \ldots \ldots \ldots$

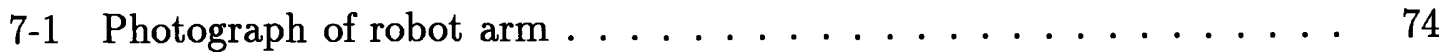




\section{List of Tables}

5.1 Characteristics of chosen motor and gearbox . . . . . . . . 40

5.2 Torsional spring Characteristics . . . . . . . . . . . . 42

5.3 Results for plate spring . . . . . . . . . . . . . . . 43

6.1 Gains for closed loop system . . . . . . . . . . . . . 60

6.2 Closed loop poles . . . . . . . . . . . . . . . . . 61 


\section{Chapter 1}

\section{Introduction}

\subsection{Approaches to Force Control}

This chapter describes briefly why force control is important for robotic manipulators. It then describes why manipulators designed using the traditional values do not perform well at this task. A novel actuator is introduced which eases some of the problems of the traditional actuators, and should allow better force control.

In order for a robotic manipulator to be of any use in the world, it needs to interact safely and controllably with it. The forces between the robot and the environment needs to be controlled so that neither the robot, nor its workpiece can damaged from normal operation, or from unexpected collisions. Some tasks, such as grinding, polishing and assembly, inherently require the control of the force between the robot and its environment.

The traditional premise for good robot design is "Stiffer is better". A stiff manipulator allows high bandwidth force control and precise position control, but the stiffness makes force control difficult. A stiff manipulator can exert high forces from small joint displacements, a large force error resulting from small errors in position. Typically the size of the joint motions to apply significant forces are comparable to the resolution of the joint angle sensors, making the control task difficult. The situation is exacerbated by the high gains used to make the positional errors small. These two factors often place the system on the brink of instability. Many researchers in force control have observed instability when applying forces, especially when contacting hard surfaces. Interestingly, they have solved this problem by wrapping compliant coverings around the endpoint of the robot to reduce its effective stiffness.

The situation is further compounded by the choice of actuator. As the robots are stiff, their links tend to be heavy, so large forces are needed to accelerate them. Electric motors, which are the most common actuator type cannot generate large forces at low speeds, so gear reductions need to be used. These increase the power density at the expense of introducing friction, noise, backlash and torque ripple to the system. As the robot is stiff, these effects will be transmitted to the endpoint of the 
robot, giving poor performance. A further disadvantage is that the gears increase the reflected inertia of the motor, and the large shock loads which result from unexpected collisions can cause the gear teeth to break.

The conclusion from this is that stiff actuators and robots are not good for force control. On the other hand, humans are good at force control, and they certainly have no problems contacting hard surfaces! The essential difference is that humans are a low stiffness, low bandwidth system, compared to the high bandwidth, high stiffness robot. This raises the possibility of sacrificing bandwidth and stiffness to achieve better, more stable force control. This can be implemented by placing an elastic element into the actuator, as illustrated in Figure 1-1.

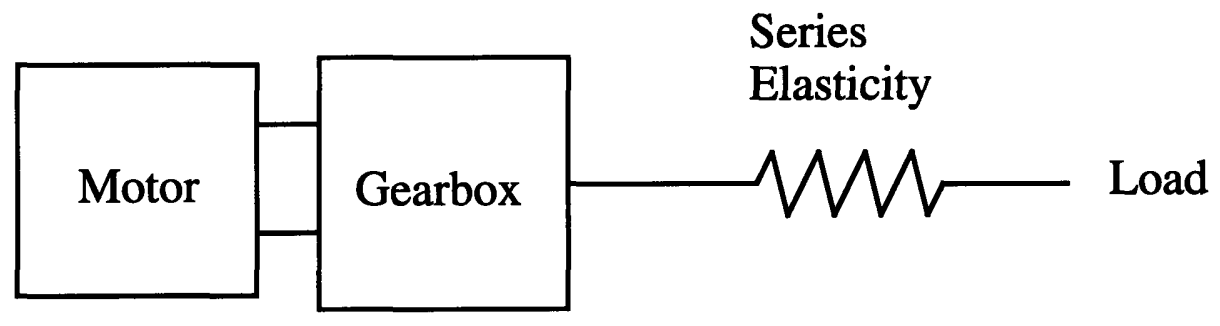

Figure 1-1: Schematic of Series-Elastic Actuator

The elasticity has the effect of making the force control easier, as larger deformations of the robot structure are needed to exert the same forces as a stiff robot. It also gives back to the motor some of the properties that were lost when gears were introduced.

The elasticity turns the force control problem into a position control one, which greatly improves force accuracy. Since the output force is proportional to the twist in the spring, the motor position determines the force. As position is more easy to control through a gear train than force, the effects of friction, backlash, and torque ripple are reduced.

The control action reduces the reflected inertia, and the elasticity low pass filters shock loads, protecting the gearbox from damage. The spring also filters the output of the actuator, limiting the bandwidth that can be achieved. As discussed above, low bandwidth control is sufficient for human-like tasks such as assembly.

Introducing series elasticity also makes stable force control easier to achieve. The motor's force feedback loop can operate well at low frequencies, so neither the motor nor the load inertia can resonate. At high frequencies, where the feedback loop no longer operates well, the system behaves like a spring, which is passive and so stable. To prevent light loads resonating on the spring a minimum mass is required, which is easily provided by the unavoidable mass of the arm.

The use of series elastic actuators should improve the performance of robots performing human-like tasks. The robot structure will be less stiff, and the force control will be less noisy, more accurate, and stable. 


\subsection{Scope of Investigation}

An actuator with a series elastic element was designed and built to evaluate some of the claims of the previous section. Theory was developed to attempt to understand the performance enhancements and limitations that the spring caused, as well as to determine a suitable control law for the system. Tests were carried out to determine how the actual actuator performed in practice.

\subsection{Review of Thesis Contents}

The thesis is organized as follows:

Chapter 2 describes some of the background literature on force control, and recent work on compliant actuators.

Chapter 3 examines a model for a series elastic actuator, and considers a control strategy. The stability of the actuator is demonstrated.

Chapter 4 considers the performance limits of an actuator with an elastic element, and compares it to a stiff actuator. The possible range of output impedances that the system can produce is analysed.

Chapter 5 describes the detailed design of a single degree actuator based on these principles, with discussion of the mechanical hardware, the motor and spring, and also the implemented control strategy.

Chapter 6 describes some experimental results for the actuator, including system identification, tuning of the control loop and assessment of the performance limits of the actuator.

Chapter 7 describes some possible applications for the actuator, in the arms of a humanoid robot, and a planetary rover.

Chapter 8 provides conclusions and recommendations for further work. 


\section{Chapter 2}

\section{Literature Survey}

This chapter reviews the literature both for force control, and early work on elastic actuators. It details different methods for achieving force control, be they placing the force control sensor at the endpoint of the robot or closing torque loops around each joint. It discusses the problems of dynamic instability under force control and some of the ways that people have dealt with the problem. Some work on controlling flexible structures, and other compliant mechanisms is discussed, and the chapter concludes by looking at the types of actuator used for robotic applications.

It is clear that to perform any task that requires interaction with the environment the force that the manipulator exerts must be controlled in some way. There are essentially two ways to do this-to provide active control of the forces, or to use a passive system such as Remote Centred Compliance (Drake [13]). The active methods have had been the focus of the bulk of robotic research in recent years. Whitney [38] gives a good review of force control methods.

The methods described by Whitney are damping control (Whitney [39]), stiffness control (Salisbury [30]), impedance control (Hogan [17]) and hybrid position and force control (Raibert and Craig [25]). These methods differ in the way that they calculate the torques to be applied at each joint, with the goal of achieving a desired endpoint force. The control of the joints may be purely feed forward, or there may be force sensors and a force control loop at each joint.

The question as to where the force should be sensed, whether at the joints or at the end effector, has been addressed by a number of authors. Cannon [8] was the first to investigate non-colocated sensors (where the sensor is not located at the actuator to be controlled). Cannon showed that colocation allows good stable control, while any significant dynamics between the sensor and the actuator can lead to instability. This is one of the reasons that manipulators have been made designed to be stiff, reducing the effect of the arm link dynamics. The advantage of wrist sensing is that a direct measurement of the force is made, rather than calculated from joint torque information. The advantage of closing torque control loops around the joints is that the system is likely to be more stable. As the torques are being controlled, the effects 
of friction, torque ripple, etc are reduced. An [1] suggested a combination of end point and joint force sensing, closing joint torque control loops to obtain stability, and using a wrist sensor to obtain good steady state force accuracy.

In different research Eppinger and Seering [14] [15] investigated the bandwidth limits on robot force control. They found that the fundamental limit on the force control bandwidth is the link stiffness. Like Cannon [8] they found that non-colocated dynamics can lead to unstable systems. They also considered the effects of simple force control laws on a model of an actuator and the environment. Volpi [36] has undertaken work to assess the performance of torque control schemes under a variety of simple controllers, using an experimentally determined transfer function.

One problem with all these schemes is that they exhibit instability while contacting stiff environments. This can result as the robot changes from position control, when it is moving freely, to force control when in contact with the environment. In addition the force control itself can exhibit instability. The force control problem has been related to a high gain position control (An [1]). The model of the manipulator and the environment is generally considered as a fourth order system which is very lightly damped, the natural frequency being related to the mass of the robot and the stiffness of the environment (Eppinger and Seering [15], An [2]). The system performance improves if the effective stiffness of the environment is reduced, for example by covering the endpoint of the manipulator with a compliant covering (Roberts [26] and Whitney [39]), or alternatively by increasing the damping on the system.

Other methods for dealing with the contact instability have been through simple control laws (Volpi [36], Yousef-Toumi [44]), or the use of nonlinear control (Xu et al. [42]) to alter the characteristics of the control system so that low gains are used when the force is moving towards the setpoint, and high when it is moving away. $\mathrm{Wu}$ and Paul used joint torque control (Wu and Paul [41]), and argued that joint force control gave stability and high performance. Marth [22] used an event based system to detect contact and deal with it. These systems depend on a controller commanding the joint torques, depending on sensed values (such as whether there is contact), so their behaviour under unexpected collisions is not good. This can happen if the collision occurs on an arm link which is not detected by the wrist force sensor. Impedance control (Hogan [17]) essentially gives the actuators a dynamic behaviour which is stable, and so unexpected collisions are dealt with more safely.

Hogan [18] and Colgate [11] proved that for any system to stable with an arbitrary environment, then it must look like a passive system.

Robot structures will always have some compliance, due to the size and weight of the links, and perhaps also due to the introduction of compliant force sensors. Work has been done on controlling the position of structures with flexibility (Cannon [9], and a review is in Balas [4]), and the position control of manipulators with flexibility at the joints (Spong [31], Lee et al [21], Chen [10]).

In recent years more work has been done on actually putting compliance into the manipulator, achieving a similar purpose to the compliant coverings mentioned 
above. Xu et al, [43] used a six degree of freedom wrist with a passive compliance and an active sensing mechanism. Hashimoto [16] used an parallel compliant mechanism. Sugano [32] used a variable rate spring to adjust the compliance of his robot finger. This work allows the manipulator compliance to be altered. However, unlike the work described in this thesis, the compliance is not controlled in these cases, position rather than force being controlled. Work on parallel elastic actuators is in progress by Morrell and Salisbury [23].

There has also been work on the choice of actuation method (Hunter at al [19]). Electric motors have been the most common type, although pneumatic and hydraulic systems have also been used (examples are Raibert [24], Waldron [37], and Conrad [12]). Most electric motors have poor power density and can only obtain high power density by operating at high speed. To accelerate or move heavy loads, step down gearboxes are used, however these introduce friction, torque ripple, noise and backlash. Harmonic drive gearboxes offer a significant improvement in backlash, but they have high friction, and non-linear stiffness effects (Tuttle [35]). The disadvantages of gears have lead some authors to use direct drive motors (Asada [3]). Salisbury [29] and Townsend [34] have had considerable success using cable transmissions, which have the advantage of not having any backlash, and reduced friction. 


\section{Chapter 3}

\section{Control and Modeling}

\subsection{Introduction}

This chapter describes a model of the actuator, and considers what control law should be used to achieve stability and good performance. The control law which was used has two main components, feedforward terms, and a servo loop, both of which are discussed in the following sections. In order to investigate the stability of the actuator while in contact with all environments, the concept of mechanical impedance is used, which is explained and justified in section 3.4.1.

\subsection{Model of Actuator}

A simple model for an actuator with a series elastic element is shown in Figure 3-1 below. Shown are the model of the motor mass $J_{m}$, the spring with stiffness $k_{s}$, the force on the motor $T_{m}$, and the output force $T_{l}$. The movement of the motor shaft, and the load are $\theta_{m}$ and $\theta_{l}$ respectively.

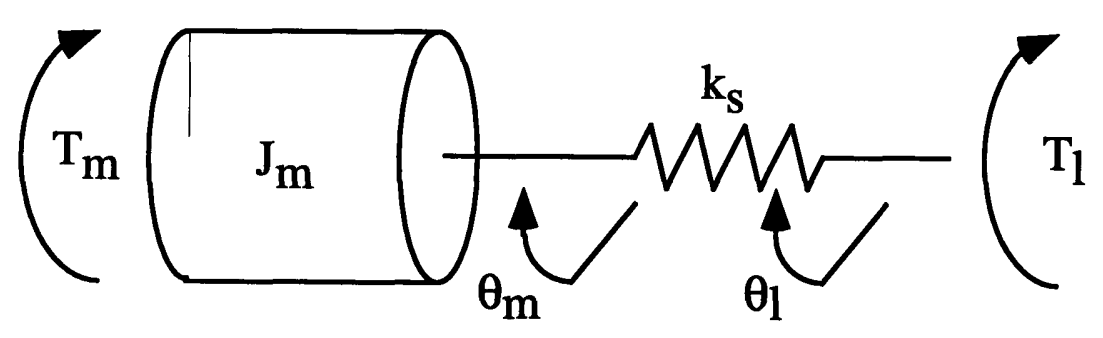

Figure 3-1: Model of actuator

Some relations are immediately forthcoming from the diagram, by applying Newton's Laws.

$$
T_{m}+k_{s}\left(\theta_{l}-\theta_{m}\right)=J_{m} \ddot{\theta}_{m}
$$




$$
-k_{s}\left(\theta_{l}-\theta_{m}\right)=T_{l}
$$

By taking Laplace transforms, an expression relating $T_{m}$ and $T_{l}$ can be found:

$$
T_{m}(s)=\left(1+\frac{J_{m}}{k_{s}} s^{2}\right) T_{l}(s)+J_{m} s^{2} \theta_{l}(s)
$$

This equation is important, as it shows what motor torques are needed to give an output torque of $T_{l}$, when the output of the actuator is moving. This equation will be considered in more detail when the performance limits of the motor are considered in Chapter 4. It also shows what the components of $T_{m}$ are, some of which can be a feedforward part of the control scheme, as described in the following section.

If the output of the actuator is assumed clamped $\left(\ddot{\theta}_{l}=0\right)$, then the transfer function between output torque, and motor torque is

$$
\frac{T_{l}}{T_{m}}(s)=\frac{1}{1+s^{2} J_{m} / k_{s}}
$$

Thus the transfer function between the actual output force $T_{l}$ and the motor force $T_{m}$ has no zeros, and two poles on the imaginary axis, at a frequency $w=\sqrt{k_{s} / J_{m}}$ which corresponds to the natural frequency of the motor mass and the spring.

The transfer function between the motion of the output shaft $\theta_{l}$ and the output force $T_{l}$ can also be written, and is shown below. The ratio $T_{l} / \theta_{l}$ is also defined as the impedance $Z$ of the system, looking from the output. The impedance will be considered in more detail in section 3.4.1, where the stability of the control system is analysed. This transfer function has the same poles as the $T_{l} / T_{m}$, but it also has two zeros at the origin. The negative sign comes from the definition of the directions of $T_{l}$ and $\theta_{1}$.

$$
Z(s)=\frac{T_{l}}{\theta_{l}}(s)=\frac{-s^{2} J_{m}}{1+s^{2} J_{m} / k_{s}}
$$

These two equations define the model of the plant to be controlled, as shown in the figure 3-2. The motion of the output shaft is modeled as a disturbance on the output torque.

\subsection{Feedforward Model}

$$
T_{m}(s)=\left(1+\frac{J_{m}}{k_{s}} s^{2}\right) T_{l}(s)+J_{m} s^{2} \theta_{l}(s)
$$

The equation above (which is 3.3 rewritten) defines how the force on the motor mass needs to vary to give an output torque $T_{l}$ while the output shaft is moving. The motor torque is made up of a component $T_{l}$ for dc behaviour, $\left(J_{m} / k_{s}\right) \ddot{T}_{l}$ to accelerate 


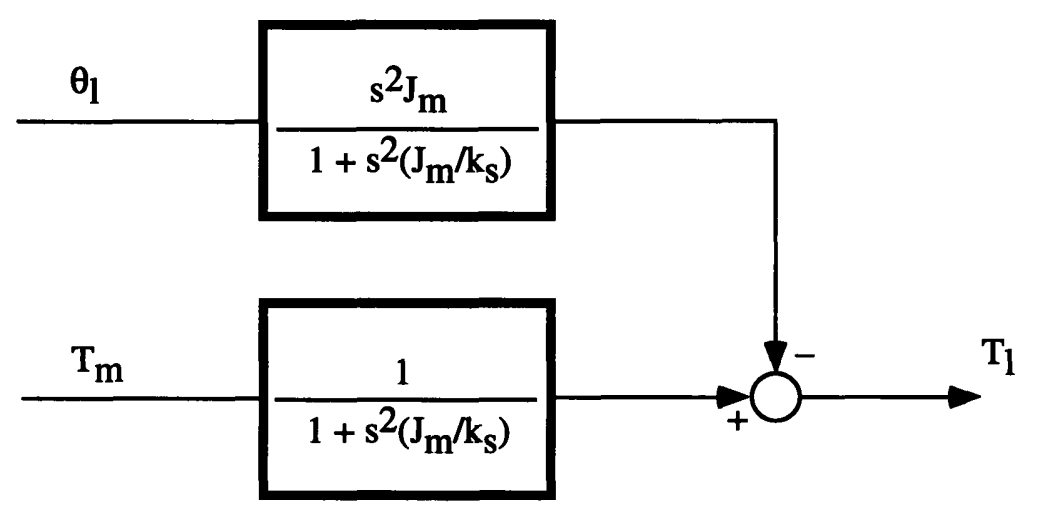

Figure 3-2: Plant model

the mass of the motor against the spring, and $J_{m} \ddot{\theta}_{l}$ to cancel out the movement of the output shaft. Alternatively the components can be viewed in terms of the wrapup of the spring, the $T_{l}$ term to provide the correct wrapup, because torque is proportional to angle , the $\left(J_{m} / k_{s}\right) \ddot{T}_{l}$ term to cancel out the effect of the motor mass vibrating on the spring, and the $J_{m} \ddot{\theta}_{l}$ term to move the motor shaft with the output shaft, maintaining the same spring wrapup.

This equation is also useful as it describes the form of the control action. By calculating the control using the equation, and using a feedback loop to compensate for errors in the model and unmodeled disturbances, a closed loop system will be obtained with better performance than one using feedback alone.

The actual feedforward model which was used on the experimental actuator is described in Chapter 5, section 5.7.

\subsection{Feedback Control Scheme}

The feedback control is required to increase the performance of the system, to compensate for errors in the feedforward model, and to reject unmodeled disturbances. It is important that the system is stable, and but perhaps more importantly, the system must be stable when in contact with all environments.

\subsubsection{Impedance and Stability}

Colgate [11] and Hogan [18] have shown that the important measure for determining whether a system will be stable while in contact with an environment is the output impedance of the system.

The impedance can be defined in a number of ways, either as the ratio of force and position $\left(T_{l} / \theta_{l}\right)$ which is used in this thesis, or the ratio between force and velocity $\left(T_{l} / \dot{\theta}_{l}\right)$. The impedance is given the symbol $Z$, and is generally a complex number, positive real impedance corresponding to mass like behaviour $\left(T_{l}=M w^{2} \theta_{l}\right)$, and 
negative real impedance to spring like behaviour $\left(T_{l}=-k \theta_{l}\right)$. A negative imaginary impedance corresponds to a damper $\left(T_{l}=-j w c \theta_{l}\right)$.

Colgate and Hogan proved that a system will be stable in contact with all environments if and only if the interaction impedance $Z(s)$, defined as $Z(s)=T_{l}(s) / \theta_{l}(s)$ obeys the following rules.

- $Z(s)$ has no poles in the right half plane $(Z(s)$ is stable)

- The imaginary part of $Z(j w)$ is negative for all frequencies $w$

These rules imply that the impedance is a stable function of frequency, and that the system is passive. A passive system is one that always absorbs energy from the environment, there never being a net transfer of energy out of the system.

\subsubsection{Proportional, Integral and Derivative Control}

The system to be controlled is second order, and so PD or PID controllers would be suitable. PD control can easily be shown to be stable, but unless the proportional gain is high, the steady state error is large. The gain cannot be raised without limit due to noise, and the sampling rate of the system controller. PID on the other hand allows better low frequency behaviour without the choice of high gains. A PID controller was tested and is evaluated below.

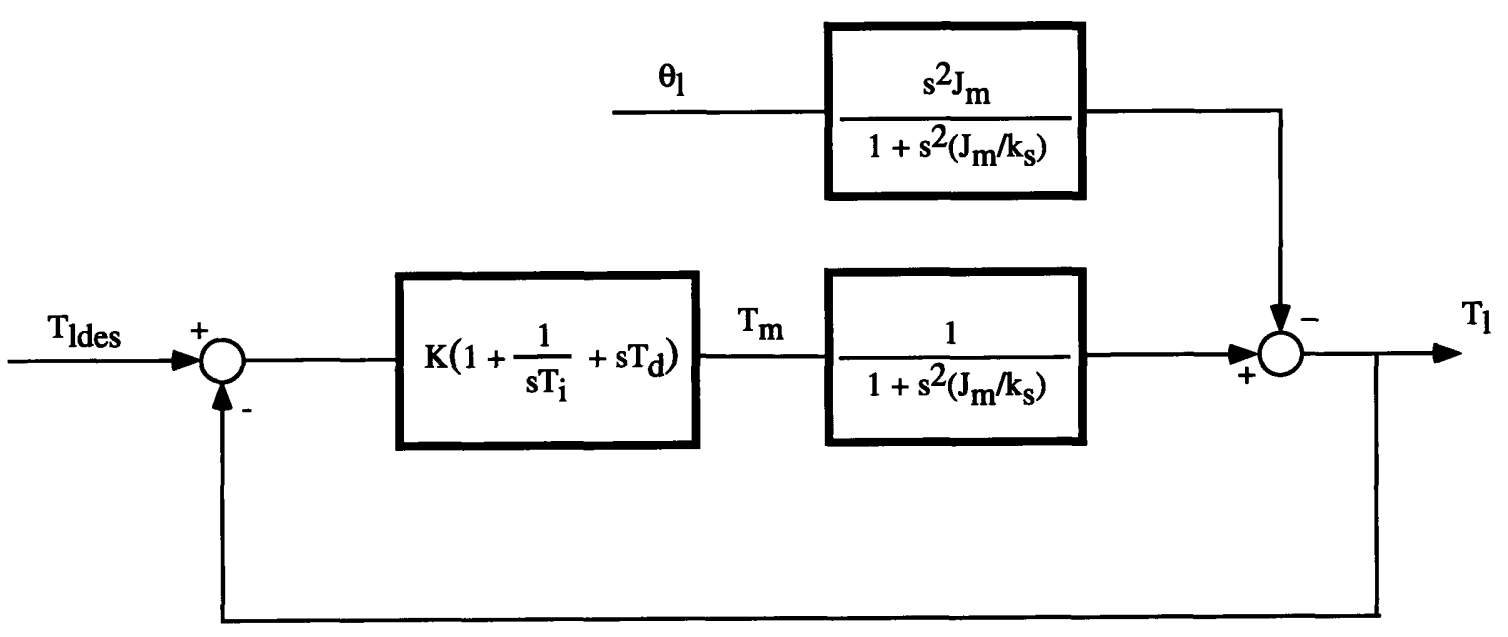

Figure 3-3: Closed loop system

Assuming that the PID is of the form $K\left(1+1 / s T_{i}+s T_{d}\right)$, then the closed loop system is illustrated in Figure 3-3, and the expression for the impedance is

$$
Z(s)=\frac{T_{l}(s)}{\theta_{l}(s)}=\frac{-s^{2} J_{m} k_{s}}{s^{2} J_{m}+k_{s}+K\left(1+s T_{d}+1 / s T_{i}\right)}
$$


So first checking that the impedance has no poles in the right half plane, considering the characteristic equation

$$
s^{3} T_{i} J_{m} / k_{s}+s^{2}\left(K T_{i} T_{d}\right)+s T_{i}(K+1)+1=0
$$

This will have roots in the left half plane if

$$
K>\frac{J_{m}}{k_{s} T_{i} T_{d}}-1
$$

so the condition for stability for all $K>0$ is

$$
1 /\left(T_{i} T_{d}\right)<k_{s} / J_{m}
$$

The system can be made stable even if this is violated by a suitably large choice of $K$. The other part of the stability proof considers the imaginary part of the impedance. Equation 3.6 rewritten with $s=j w$ is

$$
Z(j w)=\frac{T_{l}(j w)}{\theta_{l}(j w)}=\frac{w^{2} J_{m} k_{s}}{-w^{2} J_{m}+k_{s}+K\left(1+j w T_{d}+1 / j w T_{i}\right)}
$$

The only imaginary terms come from the PID term, so for the imaginary part of the denominator to be positive,

or

$$
w T_{d}-\frac{1}{w T_{i}} \geq 0
$$

$$
T_{d} T_{i} \geq \frac{1}{w^{2}}
$$

which is impossible for all $w$, since $T_{d}$ and $T_{i}$ are finite. A good way to deal with this is to roll of the integral term at low frequencies, making the controller be $K(s)=$ $K\left(1+1 / s T_{i}(1+\tau)+s T_{d}\right)$. The calculation to ensure that the poles of the closed loop system are stable is more messy for this compensator:

$$
K>\frac{J_{m}}{k_{s} T_{d} T_{i}(1+\tau)}-\frac{J_{m} \tau^{2} / k_{s}+1}{1+\tau}
$$

again, $K$ and the other time constants can be selected to make the system stable. The imaginary part of this controller is

$$
w T_{d}-\frac{w}{T_{i}\left(w^{2}+\tau^{2}\right)} \geq 0
$$

which translates to the condition on $\tau$ of

$$
w^{2}+\tau^{2} \geq \frac{1}{T_{i} T_{d}}
$$


and so the final condition on $\tau$ for stability over all $w$

$$
\tau \geq \sqrt{\frac{1}{T_{i} T_{d}}}
$$

If this condition on $T_{i}$ and $T_{d}$ is followed, then the gains for stability can be calculated using the equation above.

Unfortunately rolling off the integral term at very low frequencies gives a steadystate error, but the extra flexibility of the choice of $K, T_{i}$ and $T_{d}$ allows better closed loop performance than would be possible if the integral term was left out altogether.

Included below is a plot showing the closed loop transfer function for the case where $\sqrt{1 / T_{i} T_{d}}=11.83$. They show that when $\tau=12$ is selected as per the guideline above, the phase of the impedance is always zero or negative, while for the invalid $\tau=2$, the phase goes above zero, corresponding to non-passive like behaviour.
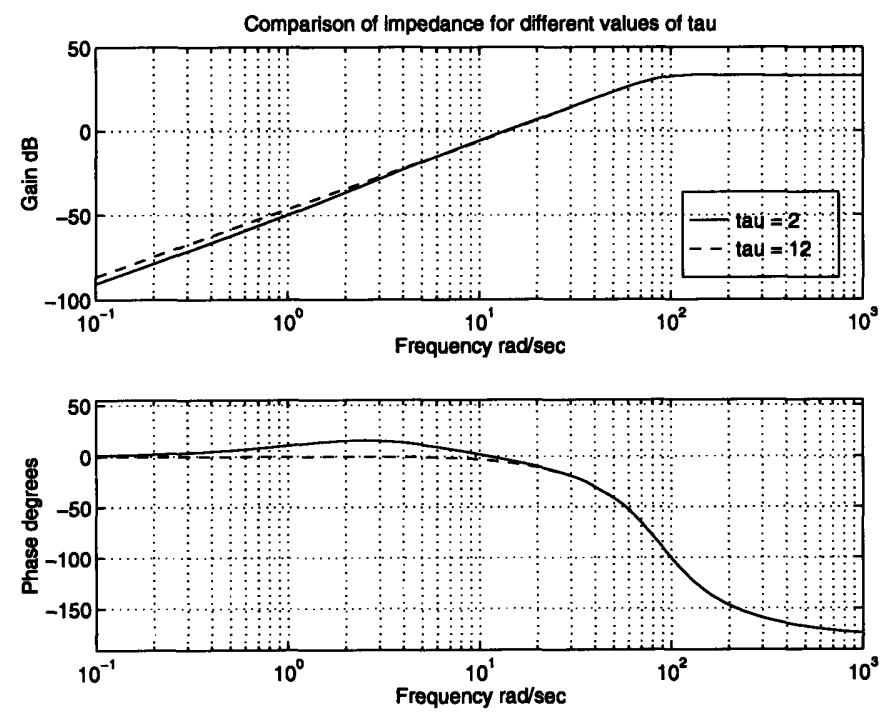

Figure 3-4: Plot of impedance for legal $\tau$ 


\subsection{Proposed Control System}

In the figure below is included a drawing of the proposed control scheme - it includes the feedforward terms and the PID control loop.

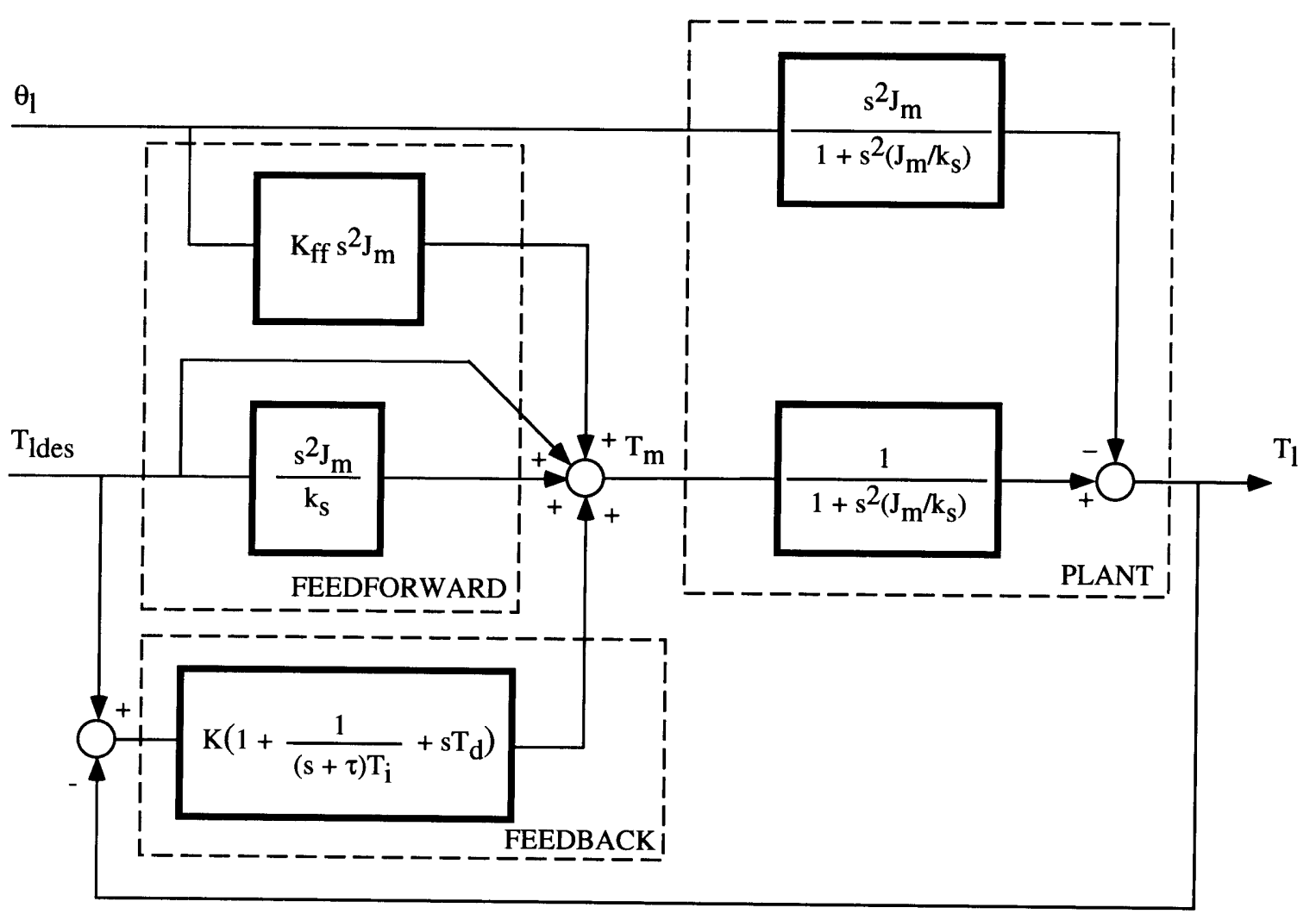

Figure 3-5: Proposed control system 


\section{Chapter 4}

\section{Performance Limits}

\subsection{Introduction}

Whenever an actuator with a series elastic element applies a certain force, the motor must move to achieve the desired wrapup of the spring. If the output shaft moves, the motor must move together with that to maintain the same force. Thus the actual motor motion is the sum of that required to generate the force, and that required to follow the output shaft. In the case of a stiff actuator, the only motion required of the motor is that of its output shaft.

As the desired force waveform and the output motion will change in size and phase during normal operation, the extra motor motion required in the elastic case may add either constructively or destructively to the actual motor motion, so either increasing or decreasing the bandwidth of the force control. This chapter considers the effect of the extra motion, and determines what effect that has on the performance of the actuator. A comparison is made between an elastic and a stiff actuator.

The treatment that follows considers a simple saturation model of the motor, where the maximum current and so the maximum torque (due to yielding in the spring, or gearbox limits) is limited. The range of forces and motions that the actuator can produce is measured subject to the limits on the motor current. This analysis is informative, and provides a good way of looking at the performance of the actuator. Also considered is voltage saturation of the motor power supply.

\subsection{Current Saturation}

In the model of the actuator described in Chapter 3, an expression was developed for how the force on the motor mass, and so the current in the motor coils depends on the output force and load motion. The equation ( 3.3 in chapter 3 ) is rewritten below.

$$
T_{m}(s)=\left(1+\frac{J_{m}}{k_{s}} s^{2}\right) T_{l}(s)+J_{m} s^{2} \theta_{l}(s)
$$


putting $s=j w$ gives the equation relating the current to the forces and motions in terms of the frequency $w$.

$$
T_{m}(s)=\left(1-\frac{J_{m}}{k_{s}} w^{2}\right) T_{l}(s)-J_{m} w^{2} \theta_{l}(s)
$$

One way to understand the meaning of this equation is to draw vectors in the complex plane representing the force $T_{l}$ and the motion $\theta_{l}$. One such figure is illustrated in Figure 4-1. In order to satisfy the constraint condition $T_{m}<T_{m a x}$, the endpoint vector $T_{m}$ must lie in the circle of radius $T_{\max }$. The $-\left(J_{m} w^{2} / k_{s}\right) T_{l}$ term always opposes $T_{l}$, so at low frequencies the elasticity moves the starting point of the $-J_{m} w^{2} \theta_{m}$ vector closer to the the centre of the circle, so allowing a wider range of forces and amplitudes than for a stiff actuator. Performance at frequencies below the natural frequency will be better when the $\theta_{m}$ vector is pointing in the same direction as the $T_{l}$ vector, corresponding to mass like behaviour, while at frequencies above the natural frequency, the system will behave better when they point in different directions, corresponding to spring like behaviour.

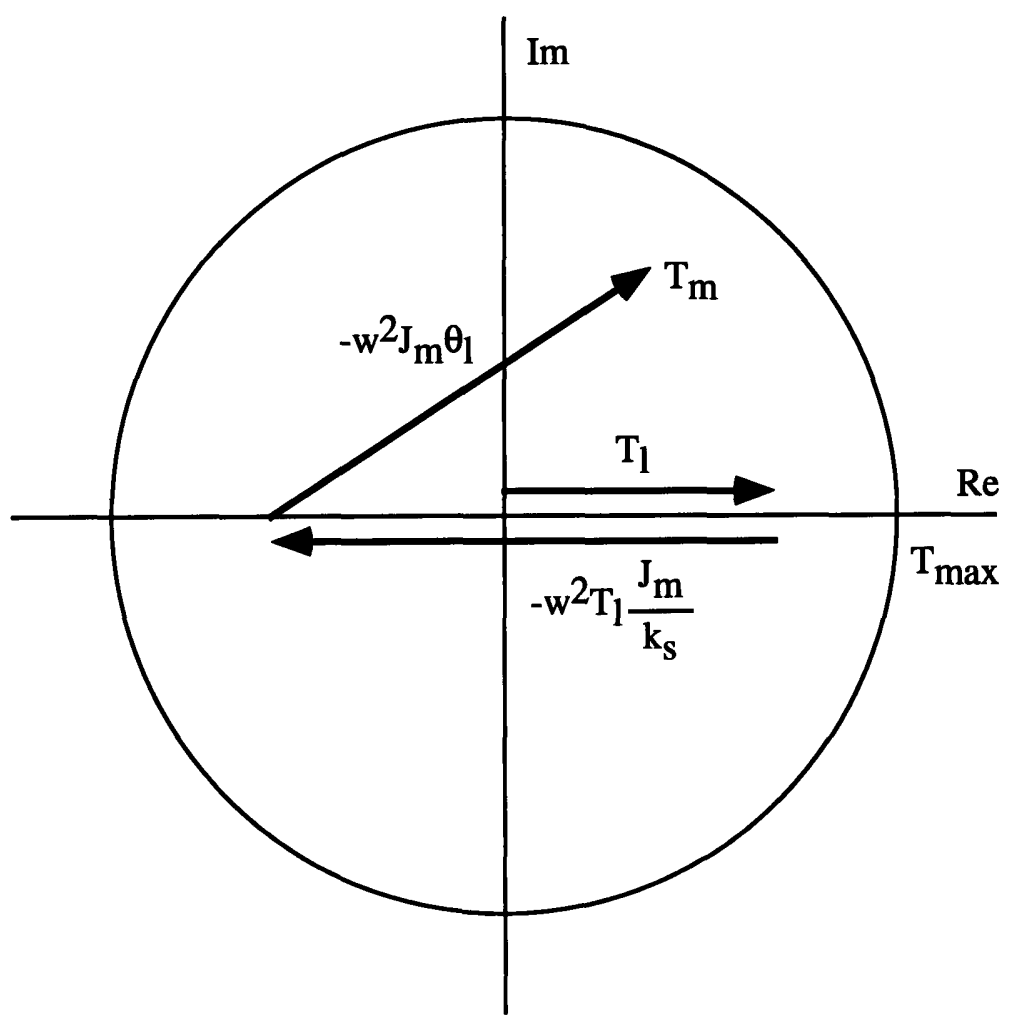

Figure 4-1: Phase diagram of motor forces

Another way to look at equation 4.1 is in terms of the impedance of the actuator, which is discussed in chapter 3 . The equation for the motor force can be rewritten in 
terms of the impedance $Z$, giving an expression for the load force:

$$
T_{l}=\frac{T_{m} Z}{\left(1+s^{2} J_{m} / k_{s}\right) Z+s^{2} J_{m}}
$$

Rewriting the equation above, and putting $s=j w$ gives:

$$
T_{l}=\frac{T_{m} Z}{\left(1-w^{2} J_{m} / k_{s}\right) Z-w^{2} J_{m}}
$$

This function has a zero at $Z=0$, and assuming that we are not at the natural frequency $\left(w=\sqrt{k_{s} / J_{m}}\right)$, a pole at $Z=J_{m} w^{2} /\left(1-w^{2} J_{m} / k_{s}\right)$ which corresponds to the natural impedance seen by the load with the motor unpowered (see equation 3.5). The zero corresponds to the difficulty of generating significant force at zero impedance (would require large or infinite motions), and the pole corresponds to the condition where force can be generated for zero motor current, so infinite force can be generated for no effort.

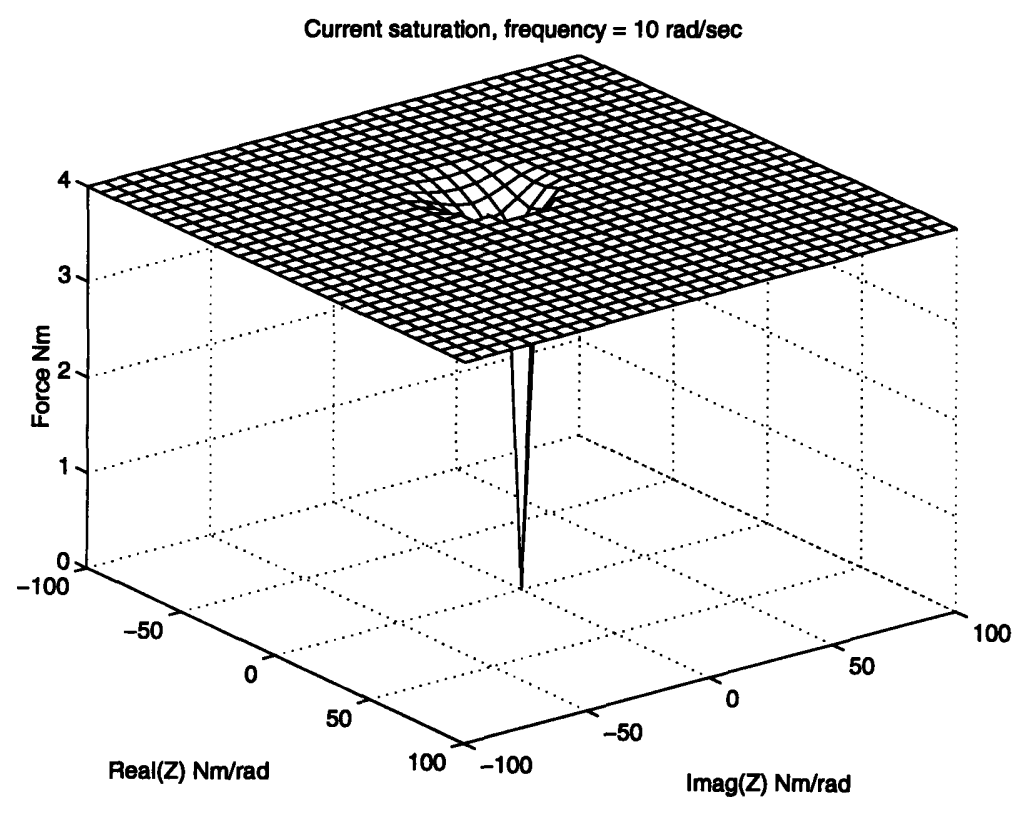

Figure 4-2: Maximum force v. impedance for below the natural frequency

Examining the characteristics of this formula in more detail show that at frequencies below the natural frequency all impedances except those near $Z=0$ may be generated at the full motor force. Indeed when $w$ is small, the spring has no effect, and it is easy to generate impedances close to the motor mass $Z=J_{m} w^{2}$. A plot of the motor force output versus impedance is shown in Figure 4-2. The pole position is not clear as the force output has been limited by the gearbox maximum torque and the spring yield point. As discussed above, generating significant force near $Z=0$ 


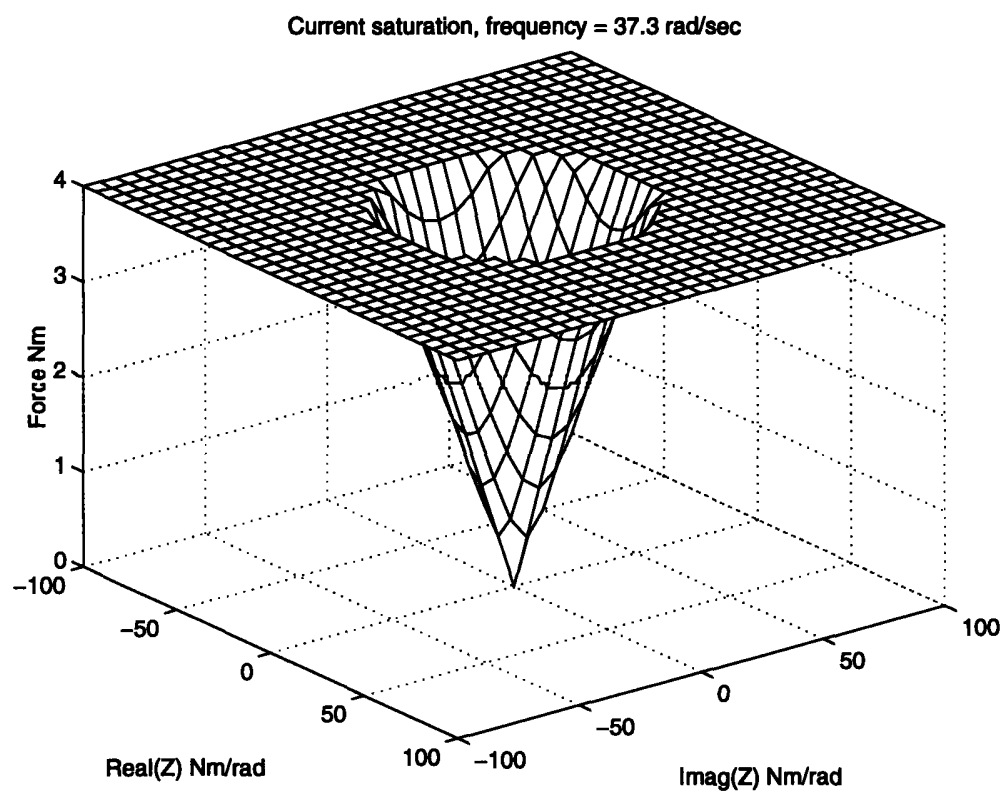

Figure 4-3: Maximum force v. impedance at the natural frequency

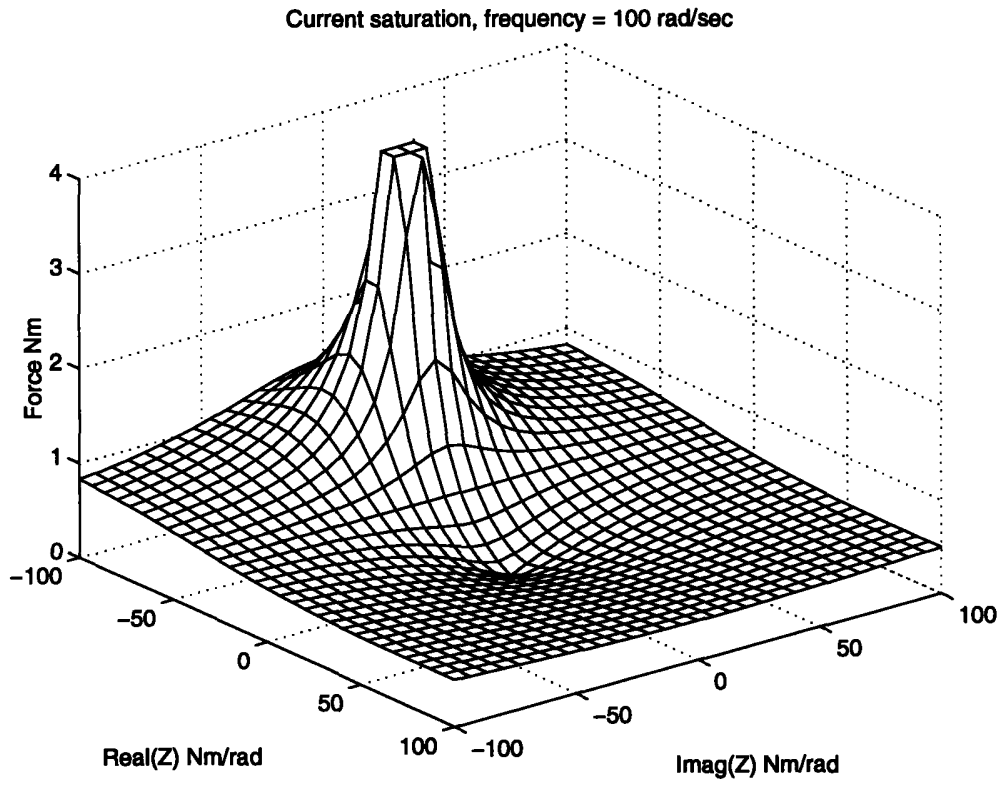

Figure 4-4: Maximum force v. impedance for above the natural frequency 
requires very large motions, and is difficult regardless of the stiffness of the actuator. At the natural frequency of the system $\left(w=\sqrt{k_{s} / J_{m}}\right)$, the pole disappears, and the ability to generate force just becomes a simple function of the impedance (see Figure 4-3). Above the natural frequency the pole reappears, and as $w$ gets large moves towards $Z=-k_{s}$, corresponding the the impedance of the spring alone (see Figure 4-4).

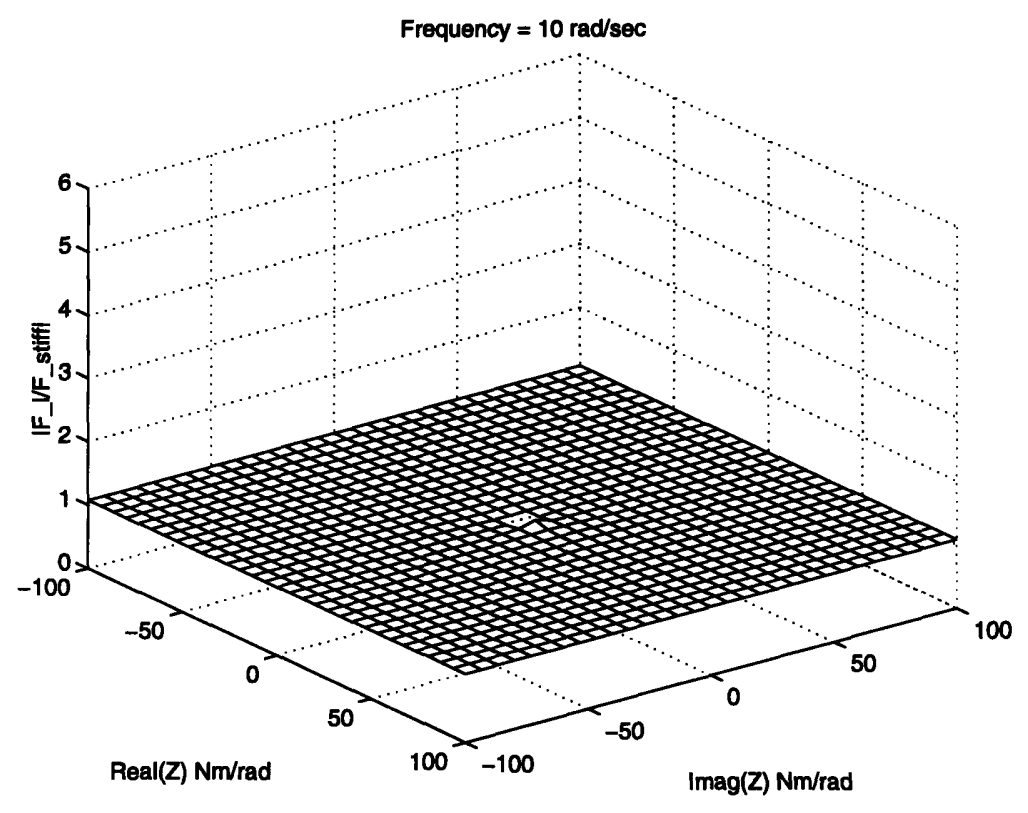

Figure 4-5: Comparison of stiff and elastic actuators at low frequency

\subsection{Comparison to a Stiff Actuator}

The equation which governs the output force of a stiff actuator as its impedance varies is similar to that described above, except that there is no pole associated with the spring:

$$
T_{l}=\frac{T_{m} Z}{Z-w^{2} J_{m}}
$$

The stiff actuator has problems generating significant force at $Z=0$, and it is particularly good at generating the impedance of its own mass $Z=J_{m} w^{2}$. The ratio of the output forces for the stiff and the elastic actuator is given by the following equation:

$$
\frac{T_{l}}{T_{l s t i f f}}=\frac{Z-w^{2} J_{m}}{\left(1-w^{2} J_{m} / k_{s}\right) Z-w^{2} J_{m}}
$$

The plots showing the behaviour of this equation are included in Figures 4-5, 4-6 and 4-7. They are similar to the previous ones except that the zero has moved from 


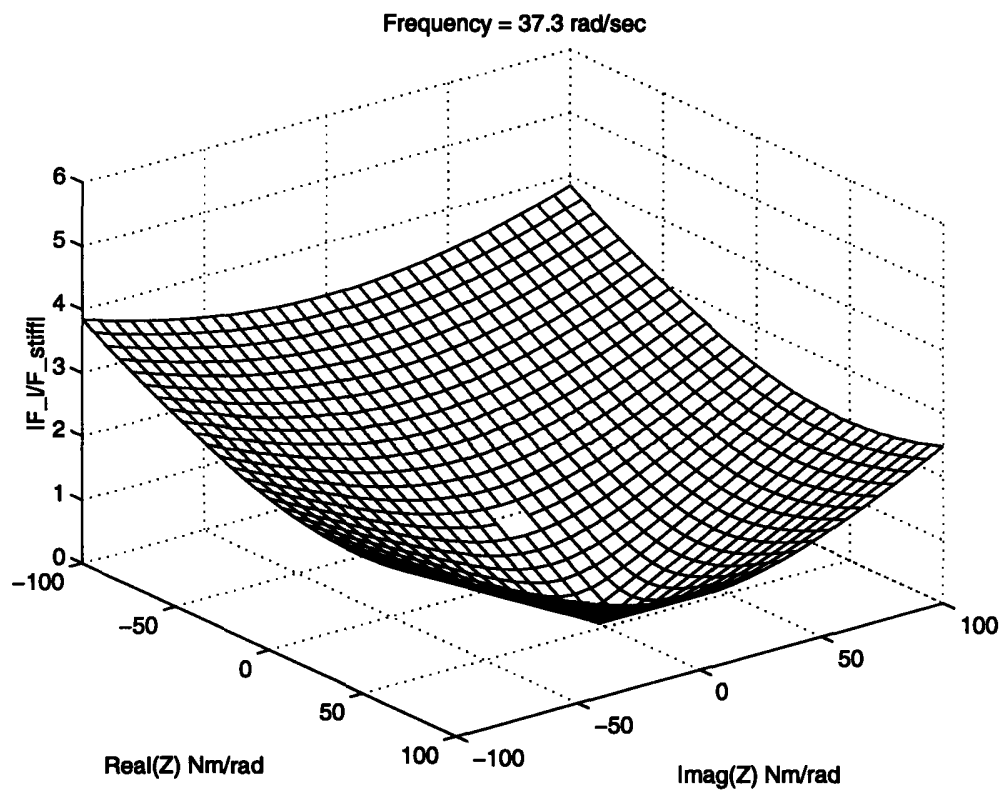

Figure 4-6: Comparison of stiff and elastic actuators at the natural frequency

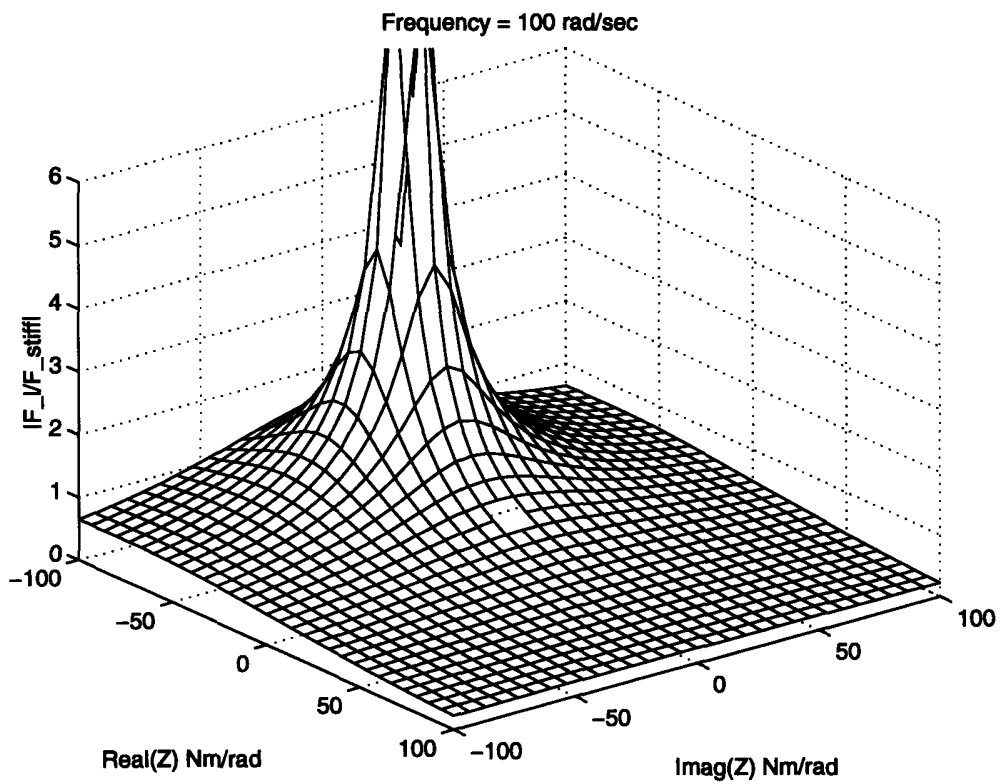

Figure 4-7: Comparison of stiff and elastic actuators at high frequency 
$Z=0$ to $Z=J_{m} w^{2}$, indicating that the series elastic actuator cannot match the unlimited performance of the stiff actuator at generating impedances close to the motor mass $\left(J_{m}\right)$, where the stiff actuator needs no power at all. The elastic actuator can generate those impedances, but they require some motor power.

At low frequencies, as shown in Figure 4-5, the performance of the stiff and the elastic actuators are the same for all impedances. As the frequency increases to the natural frequency of the elastic actuator, there is a wide range of impedances for which the elastic actuator performs better than the stiff one (see Figure 4-6). It performs better away from the impedance of the motor mass (it cannot match the unlimited performance of the stiff actuator, as discussed above). Figure 4-7 shows the comparison at high frequencies. Here the elastic actuator is behaving like a spring, and so is a lot better at generating those impedances than the stiff one. At other impedances, the stiff actuator performs better. These plots show clearly both the advantage and the penalty of introducing the spring.

\subsection{Velocity Saturation}

In order to model the effects of the motor saturation due to the voltage applied to it, the motor model in Figure 4-8 was used.

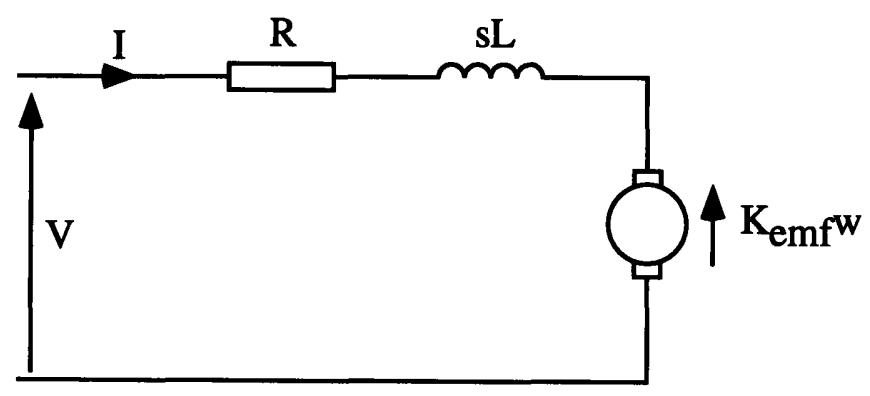

Figure 4-8: Motor model

The voltage $V$ is given by

$$
V=(R+s L) I+K_{e m f} w
$$

where $R$ is the resistance of the motor windings, $L$ their inductance, $I$ the motor current, $K_{e m f}$ the back emf constant, and $w$ the speed of the motor. The torque $T_{m}$ is related to the current by $T_{m}=K_{m} I$, so the saturation condition (equation 4.1) can be written

$$
V=\frac{(R+s L)}{K_{m}}\left(T_{l}\left(1+s^{2} J_{m} / k_{s}\right)+s^{2} J_{m} \theta_{l}\right)+s K_{e m f} \theta_{m}
$$


from equation $3.2, \theta_{m}$ can be written as

$$
\theta_{m}=\theta_{l}+\frac{T_{l}}{k_{s}}
$$

from which it follows that

$$
V=T_{l}\left[\frac{(R+s l)}{K_{m}}\left(1+s^{2} J_{m} / k_{s}\right)+\frac{K_{e m f} s}{k_{s}}\right]+\theta_{l}\left[\frac{(R+s L)}{K_{m}}\left(s^{2} J_{m}\right)+K_{e m f} s\right]
$$

or expressing as a function of the impedance $Z$, and writing $s=j w$

$$
T_{l}=\frac{V Z}{Z\left[\frac{(R+j w L)}{K_{m}}\left(1-w^{2} J_{m} / k_{s}\right)+j w\left(K_{e m f} / k_{s}\right)\right]-w^{2} J_{m} \frac{(R+j w L)}{K_{m}}+j w K_{e}}
$$

This is similar to the previous limit except that it is more difficult to visualize what is going on due to the imaginary terms. Figures 4-9, 4-10 and 4-11 show the performance limits as before.

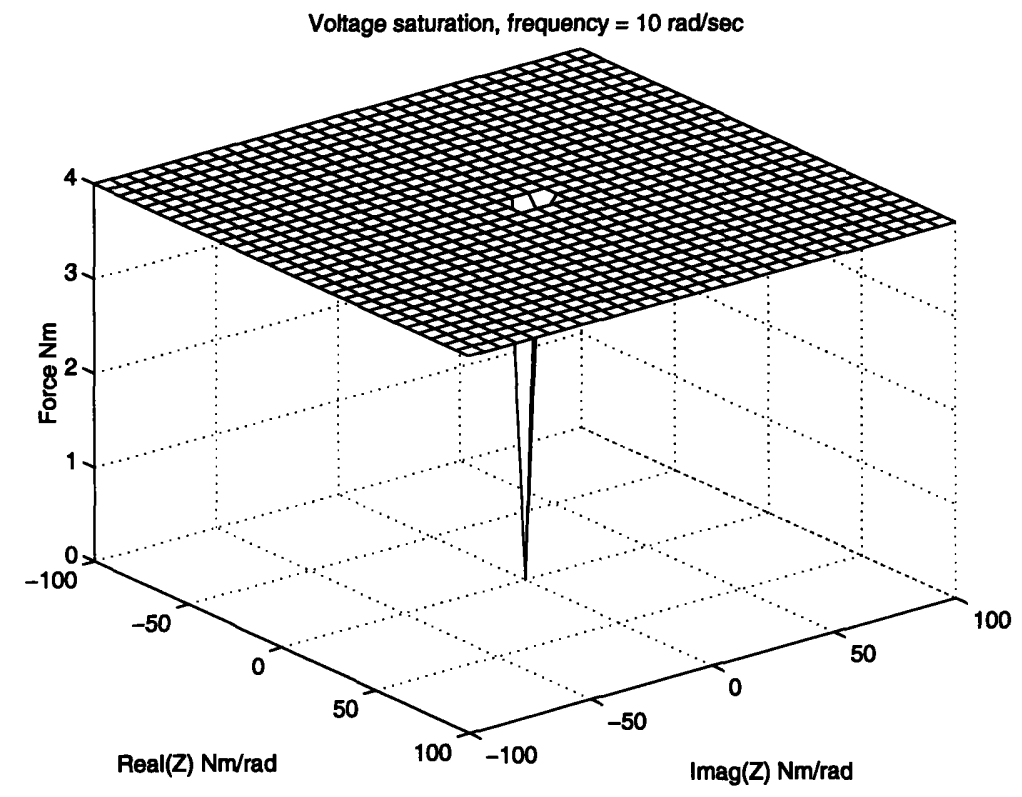

Figure 4-9: Velocity saturation below the natural frequency

At low frequencies (Figure 4-9) there is no velocity saturation except at very low impedances (where as discussed before there are large motor motions). Figure 4-10 shows a plot at the natural frequency of the system. The effect of the spring is to make the motor motion greater for impedances with negative real parts than for those with positive ones, giving a velocity limit over the mass-like part of the impedance diagram. The back emf is dependent on the velocity of the motor motion, which corresponds to a rotation of $\pi / 2$ on the impedance plot. Thus the velocity saturation 


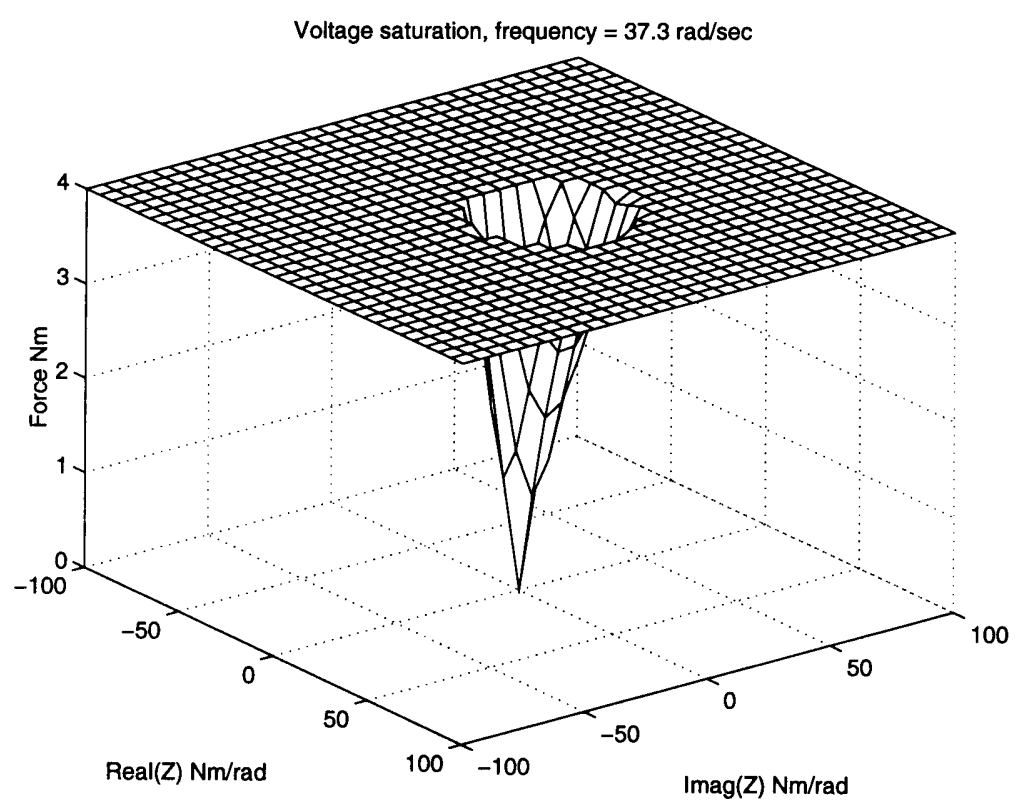

Figure 4-10: Velocity saturation at the natural frequency

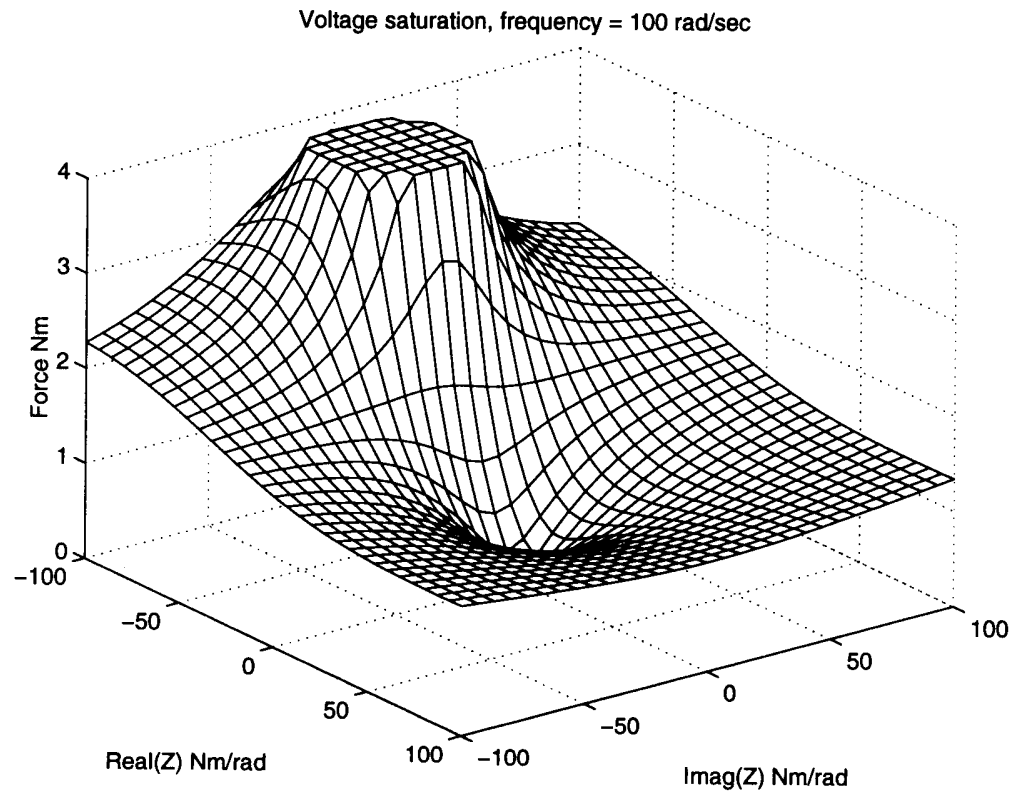

Figure 4-11: Velocity saturation above the natural frequency 
graph is not symmetric about the real axis.

Figure 4-12 shows the difference in the movement of the output shaft of the motor for a stiff actuator, and the experimental actuator. It indicates very well both the advantage and the penalty of using the spring, for impedances with a positive real part, corresponding to spring like behaviour, the elastic actuators motor moves less than the stiff one, thus higher forces can be generated. In the other half of the impedance plane, the elastic actuator has to move more than the stiff one, as so its performance is less good.
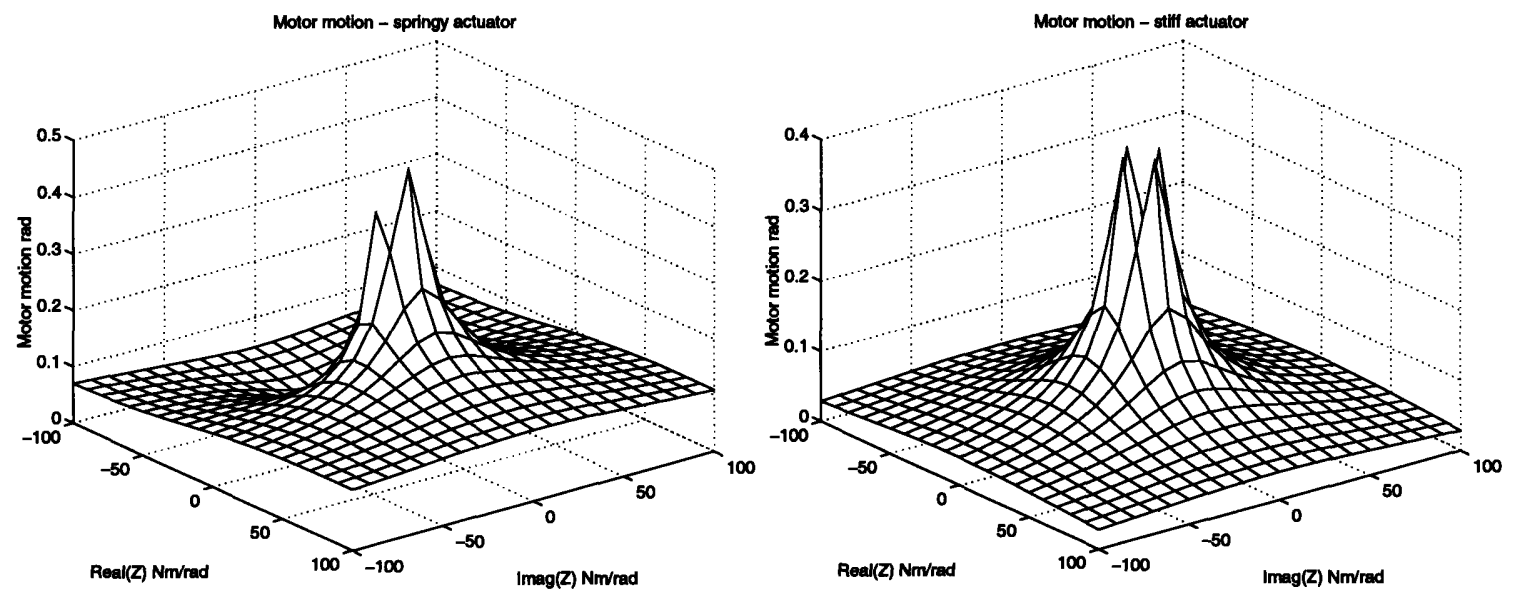

Figure 4-12: Motor movement for a stiff and an elastic actuator

At high frequencies (Figure 4-11), the only place where the motor does not saturate is where the system is acting like a spring, where the motor motion is small.

\subsection{Discussion}

For the particular parameters of the motor chosen for this application (see chapter 5), the current limit is more severe than the voltage limit. This is because the actual output torque is limited by the strength of the gearbox teeth, and the yield strength of the spring, rather than the maximum current. Indeed the theoretical maximum torque (stall torque times gearbox ratio, assuming that everything is perfectly efficient) is approximately four times the strength of the gearbox teeth. This factor gives a lot of leeway on the voltage, and makes the voltage saturation not important in this case. In other applications, it may well become significant. 


\section{Chapter 5}

\section{Actuator Design}

\subsection{Introduction}

This chapter describes the build of a series elastic actuator. The specification for the actuator was determined by its eventual application as part of the arm for the humanoid robot $\operatorname{Cog}[7]$. This application is described in Chapter 7. Figure 5-1 shows a photograph of the final actuator design. It consists of an electric motor and a spring instrumented with strain gauges. The actuator has a maximum output torque of $4 \mathrm{Nm}$. The following sections of this chapter describe in detail the choice of motor, the design of the series spring, the control hardware that was used, as well as the control algorithms implemented on the actuator. Chapter 6 describes the experimental results obtained with this actuator.

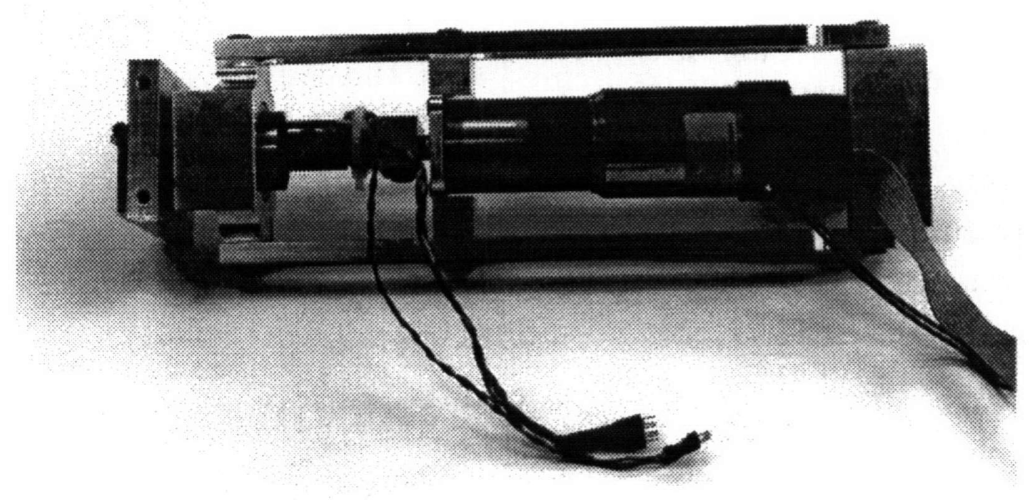

Figure 5-1: Photograph of actuator 


\subsection{Choice of Motor}

The considerations which were taken into account while choosing the motor and the gearbox were the power of the motor, the expected output speed of the motor-gearbox system under full load, and the maximum allowable output torque. Motors from a number of manufacturers were considered, using a spreadsheet to perform the design calculations. Details of the chosen motor are given below.

\begin{tabular}{||l|l||}
\hline \multicolumn{2}{|c||}{ Motor-MicroMo 3557K } \\
\hline Power & $25 \mathrm{~W}$ \\
\hline Voltage & $48 \mathrm{~V}$ \\
\hline Torque constant & $0.086 \mathrm{Nm} / \mathrm{Amp}$ \\
\hline Stall Torque & $0.177 \mathrm{Nm}$ \\
\hline No load speed & $5200 \mathrm{rpm}$ \\
\hline Weight & $275 \mathrm{~g}$ \\
\hline Efficiency & 0.74 \\
\hline Inertia Gearbox-MicroMo $30 / 1$ \\
\hline \multicolumn{2}{|c||}{$4.59 \times 10^{-6} \mathrm{Kgm}^{2}$} \\
\hline Reduction & $66: 1$ \\
\hline Continuous maximum torque & $1.8 \mathrm{Nm}$ \\
\hline Intermittent maximum torque & $2.4 \mathrm{Nm}$ \\
\hline Efficiency & 0.60 \\
\hline Gearbox weight & $171 \mathrm{~g}$ \\
\hline Expected speed with 4Nm load & $27.0 \mathrm{rpm}$ \\
\hline
\end{tabular}

Table 5.1: Characteristics of chosen motor and gearbox

It was found that the main limiting factor in the choice of the gearbox was the strength of the output teeth. A safety factor of 1.5 was used in the design calculations, after representatives of MicroMo confirmed that the data was conservative.

The motor was purchased with an encoder HEDS 5010, to allow the position of the motor output shaft to be measured accurately. The number of lines required (360) was determined by the reduction ratio of the gearbox, and encoder decoding circuitry that was used (16 bit).

\subsection{Spring Design and Development}

The design of the spring was by far the most difficult part of the design. As the spring is in series with the output and carries the full actuator load, it is important that it will not yield under normal working conditions. In addition its stiffness must be low enough to exhibit flexibility at these loads. As will be seen in this section, it is difficult to design a spring which has at the same time a high yield strength and 
low stiffness. The design targets for the spring were $4 \mathrm{Nm}$ maximum torque, with an angle of twist of about 5 degrees.

The design of the spring also motivated the mechanical layout of the actuator. It was felt that a simple actuator would be best to build at first, and so cables and tendons were avoided. The design chosen is illustrated in Figure 5-2. A torsional spring is used as the elastic element, mounted between the motor shaft and the actuator output. The output runs in a bearing, the spring passing through the centre of the axle to save space. The gearbox has bearings which support the other end of the spring. Arranging the spring like this allows a compact design, but puts a limit on the maximum diameter of the spring of about 0.9 " $(23 \mathrm{~mm})$, due to the availability of bearings. The maximum length of the spring was set to $3 "(76.2 \mathrm{~mm})$ to further ensure the compactness of the design.

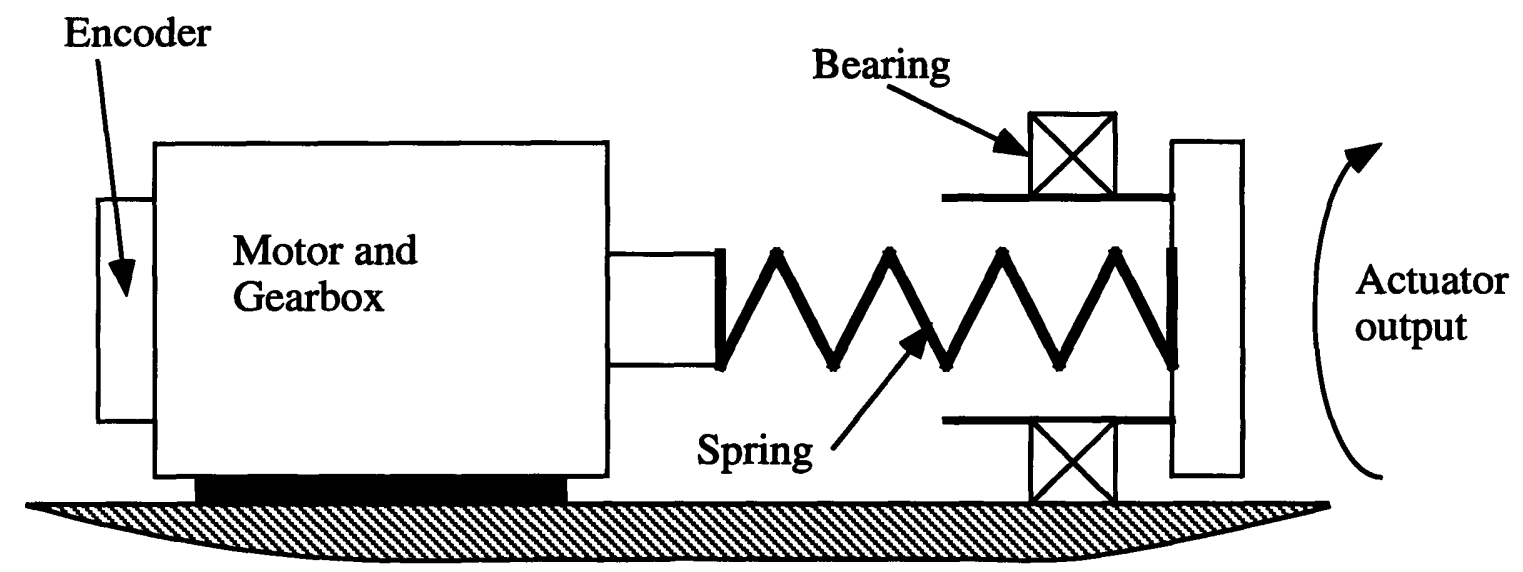

Figure 5-2: Schematic of mechanical design

The most obvious type of torsional spring is a cylinder. For a cylinder with length $l$, diameter $d$, the formula for the maximum shear stress under torsion is [27]

$$
\tau_{\max }=\frac{16 T_{\max }}{\pi d^{3}}
$$

Where $T_{\max }$ is the maximum force. This can be rearranged to show that the minimum diameter for the rod is

$$
d_{\text {min }}=\sqrt[3]{\frac{32 T_{\max }}{\pi \sigma_{\text {yield }}}}
$$

using Tresca's yield criterion, that yielding begins when $\tau_{m a x}$ is equal to half of the yield stress, $\sigma_{\text {yield }}$. The expression for the angle of twist of the rod at yield, the minimum length of the rod (given that angle), and the resulting stiffness of the spring are given below, where $G$ is the shear modulus.

$$
\theta_{\text {yield }}=\frac{32 T_{\max } l}{\pi d^{4} G}
$$




$$
\begin{aligned}
l_{\min } & =\frac{\pi d_{\min }{ }^{4} G \theta_{\text {yield }}}{32 T_{\max }} \\
k_{\text {spring }} & =\frac{\pi d_{\min }{ }^{4} G}{32 l}
\end{aligned}
$$

The table below show parameters for springs of this type made from a number of different materials, for the design condition $T_{\max }=4 \mathrm{Nm}, \theta_{\text {yield }}=5$ degrees.

\begin{tabular}{||l|r|r|r|r|r||}
\hline Material & $E \mathrm{kN} / \mathrm{mm}^{2}$ & $G \mathrm{kN} / \mathrm{mm}^{2}$ & $\sigma_{\text {yield }} \mathrm{N} / \mathrm{mm}^{2}$ & $d_{\min } \mathrm{mm}$ & $l_{\min } \mathrm{mm}$ \\
\hline Steel & 210 & 81 & 240 & 5.54 & 163.4 \\
Aluminium & 70 & 27 & 200 & 5.88 & 69.1 \\
Delrin & 3.6 & 1.4 & 69 & 8.39 & 14.9 \\
Nylon 66 & 3.3 & 1.27 & 81.6 & 7.93 & 10.75 \\
\hline
\end{tabular}

Table 5.2: Torsional spring Characteristics

Of the materials in the table, steel is probably the most appropriate, as it has a definite yield point. Most commercially available springs are made from steel (spring steel). However, in this configuration the use of steel is rather impractical. Aluminium is more practical, but it does not have a definite yield point. The plastics are also promising, but they have a stock of undesirable properties, such as creep, hysteresis and temperature effects, in addition to the mechanical problems associated with gripping them.

The problem with cylindrical springs is that they are too efficient in torsion-if they are designed so that they will not yield, they are too stiff. Other possible sections were considered were tubes and square sections, which were also too efficient, and flat plates which were more promising.

The equations which define the torsional behaviour for a flat plate (such as in Figure 5-5 (b)), with length $l$, width $b$ and thickness $t$ are given below [5]

$$
\begin{aligned}
\tau_{\max } & =\frac{t_{\max } G \theta}{l_{\min }} \\
T & =\frac{1}{3} b t_{\max }^{3} \frac{G \theta}{l_{\min }} \\
k_{\text {spring }} & =\frac{b t_{\max }^{3} G}{3 l_{\min }}
\end{aligned}
$$

Equation 5.6 shows that the yield angle is determined by the ratio $t_{\max } / l_{\min }$ and is independent of the width $b$. The stiffness of the spring (and the maximum torque) follow from the choice of the width. This is good as the spring yield angle and the stiffness can be designed separately. The table below shows designs for a flat plate spring with length $3 "(76.2 \mathrm{~mm})$.

The values for $b$ are large, especially for steel which would make the use of this type of spring difficult in practice. However, if the plate is cut into strips, and arranged as 


\begin{tabular}{||l|r|r|r|r|r|r||}
\hline Material & $E \mathrm{kN} / \mathrm{mm}^{2}$ & $G \mathrm{kN} / \mathrm{mm}^{2}$ & $\sigma_{\text {yield }} \mathrm{N} / \mathrm{mm}^{2}$ & $l / t$ & $t_{\min } \mathrm{mm}$ & $b_{\min } \mathrm{mm}$ \\
\hline Steel & 210 & 81 & 240 & 58.9 & 1.29 & 60.4 \\
Aluminium & 70 & 27 & 200 & 23.6 & 3.23 & 11.5 \\
\hline
\end{tabular}

Table 5.3: Results for plate spring

shown in Figure 5-3, then the wide sheet can be packed into a compact spring. As the yield angle is determined by $t$ and $l$, the stiffness can be altered by simply adjusting the width and the number of strips. The equations will no longer hold precisely as not all the strips pass through the axis of twist.

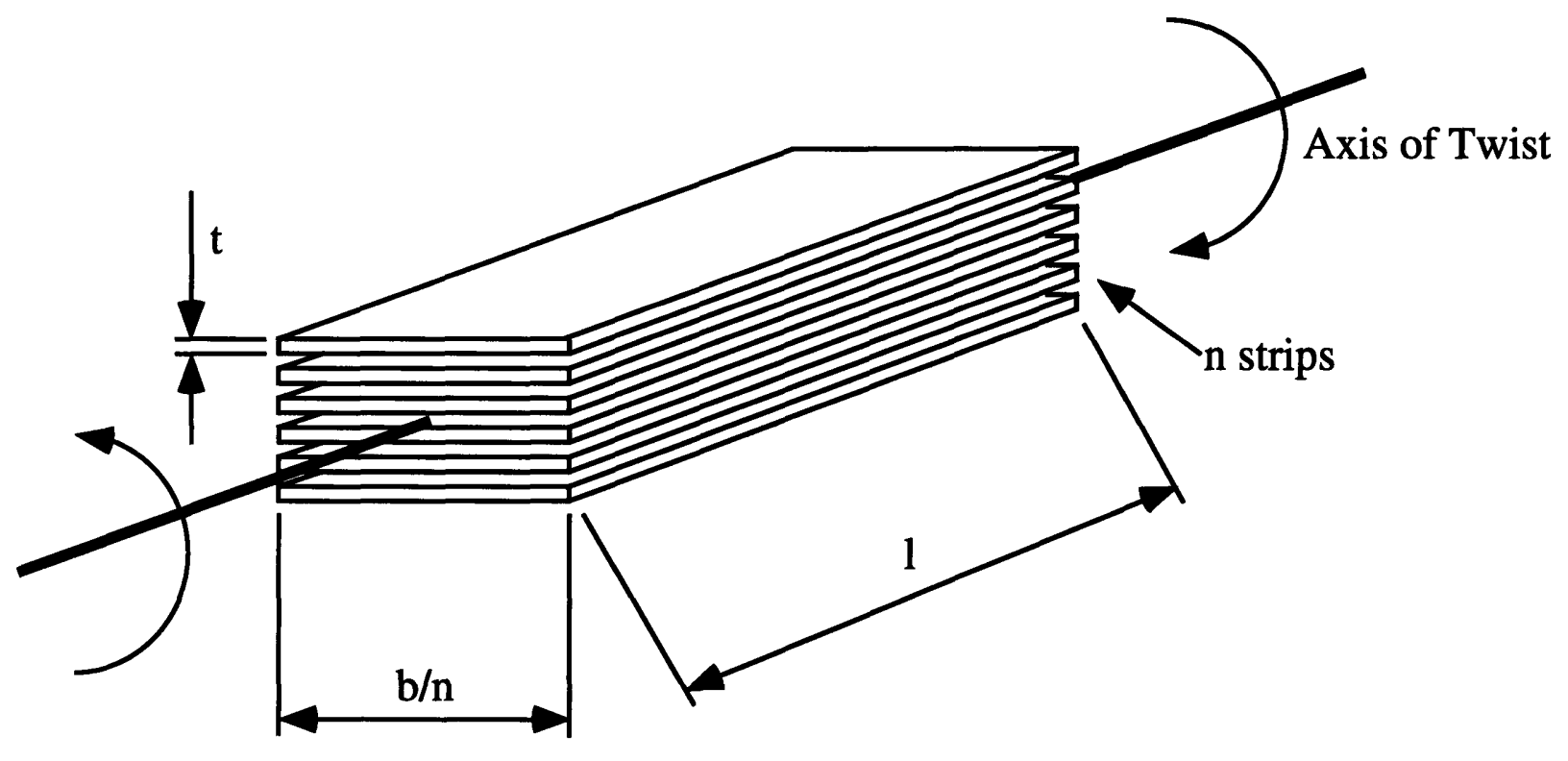

Figure 5-3: Composite spring drawing

A spring of this design was built out of strips of shim steel (hardened steel) fastened with clamps. A good design was used 6 strips, of width 0.55 " $(14 \mathrm{~mm})$, length $1.9 "(48.3 \mathrm{~mm})$, and thickness $0.032 "(0.81 \mathrm{~mm}) .0 .01 "(0.25 \mathrm{~mm})$ spacers were placed between the sheets to prevent them rubbing against each other. The spring was found to work reasonably well, the force being roughly linear with the twist angle, with a small amount of hysteresis, as shown in Figure 5-4. The spring was quite a lot stiffer than predicted $(40.1 \mathrm{Nm} / \mathrm{rad}$ as opposed to $25.1 \mathrm{Nm} / \mathrm{rad})$ which is probably a consequence of the outermost spring layers being in a combination of tension and torsion, rather than pure torsion. Early experiments were carried out performing torque control using a spring of this design.

The composite spring was difficult to manufacture, assemble and maintain, the main problem being fastening the strips securely. It was also lossy due to the hysteresis, so a better one piece spring was devised. By selecting a cross shape, more strips could be put in pure torsion, as shown in Figure 5-5 (a). A photograph of a typical 


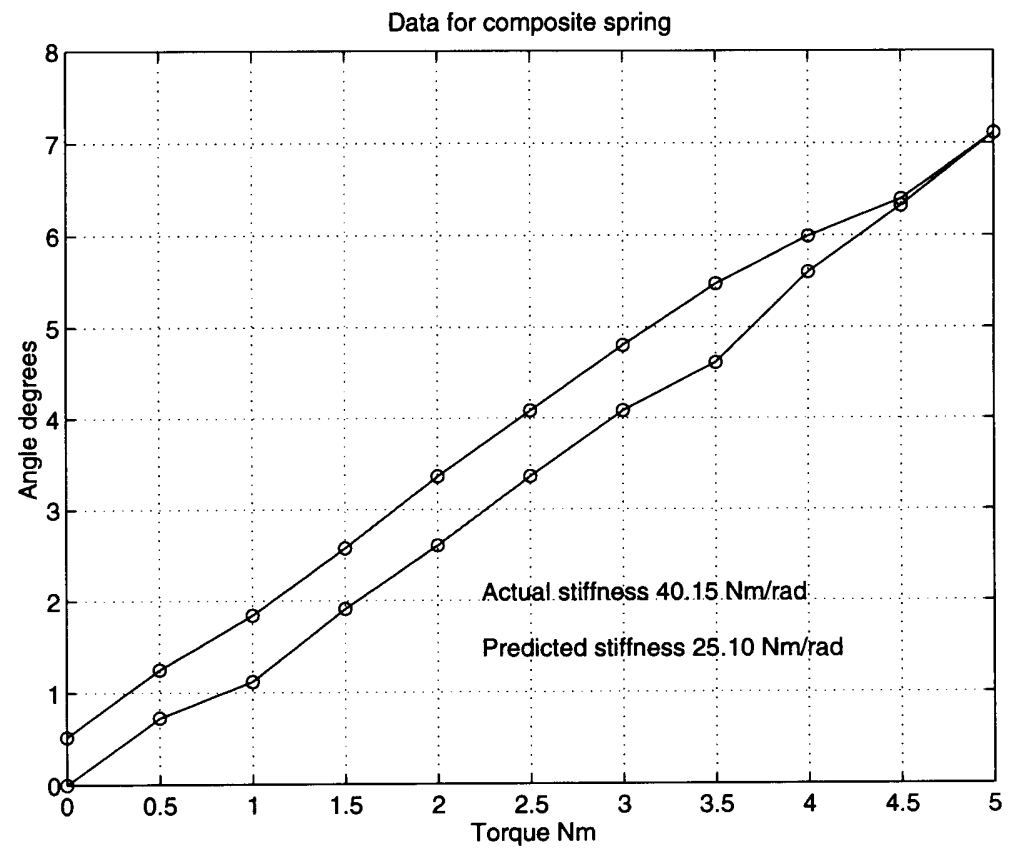

Figure 5-4: Results for bar spring (6 strips)

spring is included in Figure 5-6.

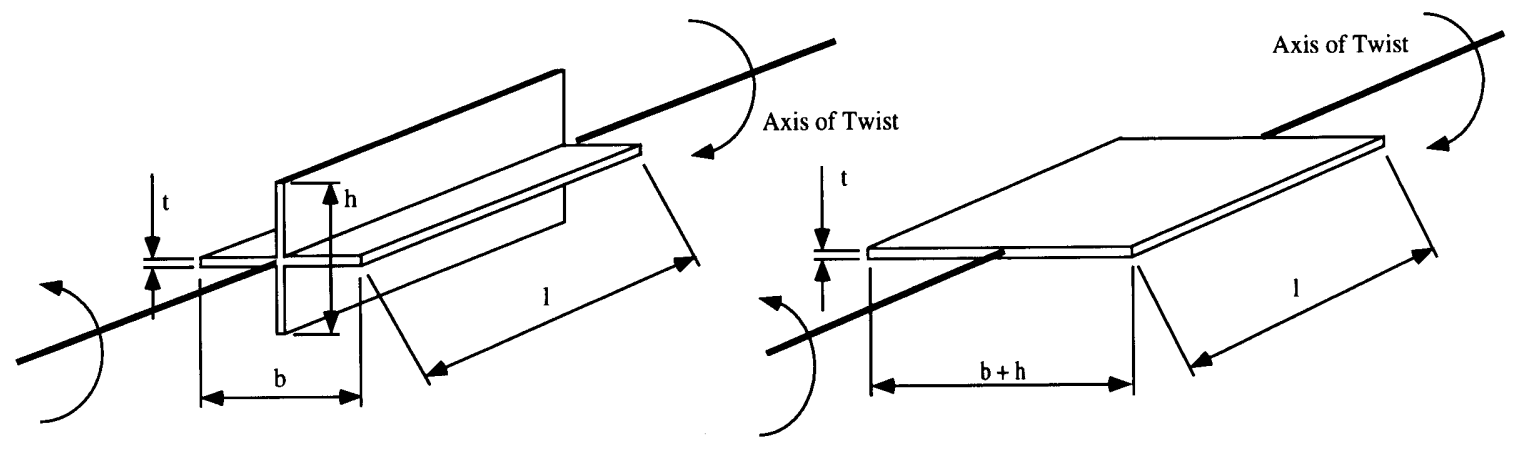

(a) Cross Spring

(b) Equivalent flat plate

Figure 5-5: Cross Spring Drawing

It was not obvious how to relate the torsional behaviour of this type of spring to the flat plate, so a number of springs were machined and their performance was compared to that of a flat plate of equivalent dimensions (see Figure 5-5). The springs had different web thicknesses $(t)$, lengths $(l)$, spring sizes $(b, h)$, and were manufactured from either aluminium, or pre-hardened steel (AISI/SAE 4142). The yield angle, maximum torque and stiffness was evaluated for each of the springs. The flat plate behaviour was calculated using the equations below, which are taken from 


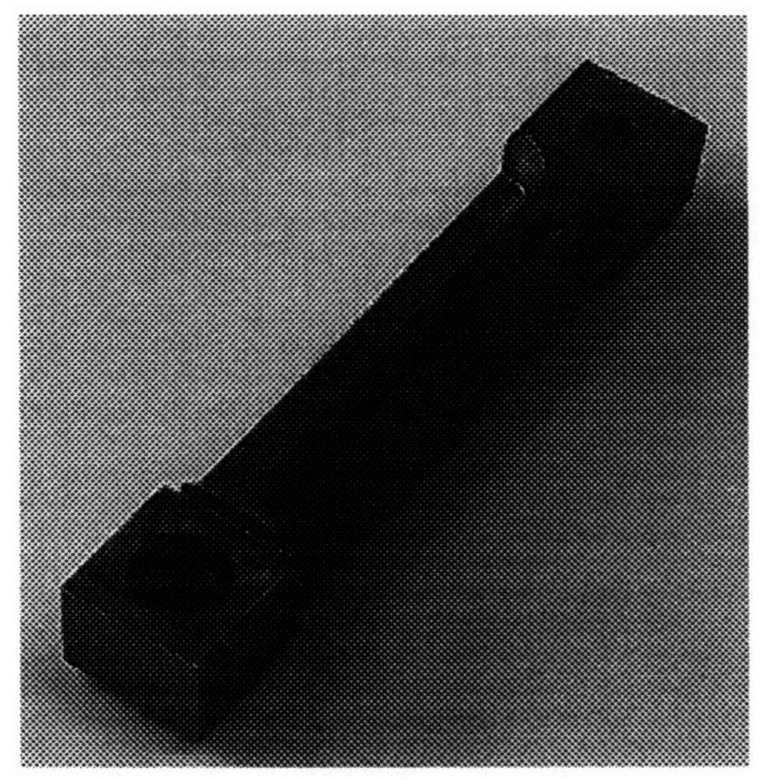

Figure 5-6: Cross spring photograph

equations 5.6 and 5.8 .

$$
\begin{aligned}
\theta_{\text {max }} & =\frac{l}{t G \tau_{\max }} \\
k_{\text {spring }} & =\frac{(b+h) t^{3} G}{3 l} \\
T_{\max } & =k \theta_{\max }
\end{aligned}
$$

Figure 5-7 shows a comparison between the stiffness of the cross spring and the flat plate. The relationship is a straight line which shows that the cross spring's behaviour obeys the same general rules as the flat plate. There is a scaling difference; if the cross spring behaved in exactly the same way as an equivalent flat plate the gradient of the line would be 1.0 , while it is actually 0.8 . This means that the cross spring section is inherently less stiff than the flat plate.

This is quite surprising given that the joint between the two webs would be expected to make the spring stiffer. However the spring material is a lot nearer to the twist axis in the cross shaped case than for the flat plate, which will reduce the stiffness.

Figure 5-8 shows a similar comparison for the maximum angle of twist. Again the results support the conclusion that the cross spring is behaving the same as the flat plate with a scaling factor difference. This time the maximum angle of twist is greater for the cross spring, the factor being around 1.76. The reasons for this difference are unclear, especially since the theory suggests that the maximum twist angle is independent of the width $b$, so the proximity of the webs to the axis should 


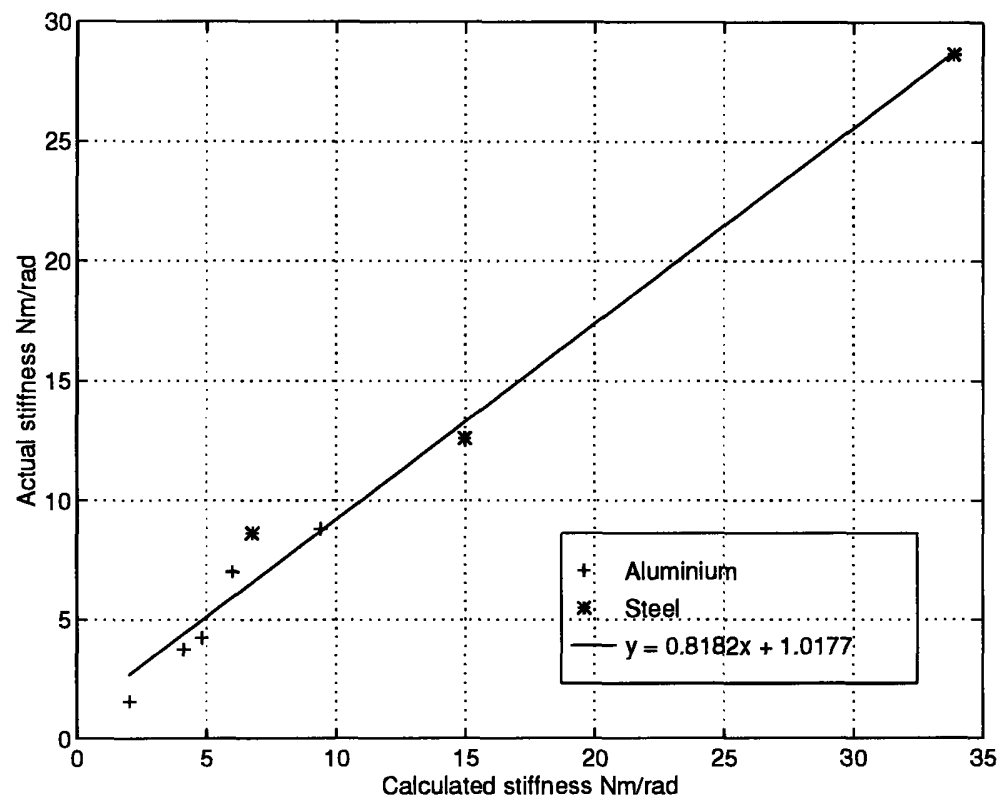

Figure 5-7: Comparison of cross spring stiffness

make no difference to the yield angle.

The stiffness and the maximum angle determine the maximum torque that can be carried by the spring. Figure 5-9 shows clearly the advantage of the cross shape. Although the stiffness of the section is less than predicted, the increased angle of twist outweighs that and makes the maximum torque about 1.6 times as high as for the flat plate. The increased torque together with the compact design makes this type of spring most suitable for use in the actuator. 


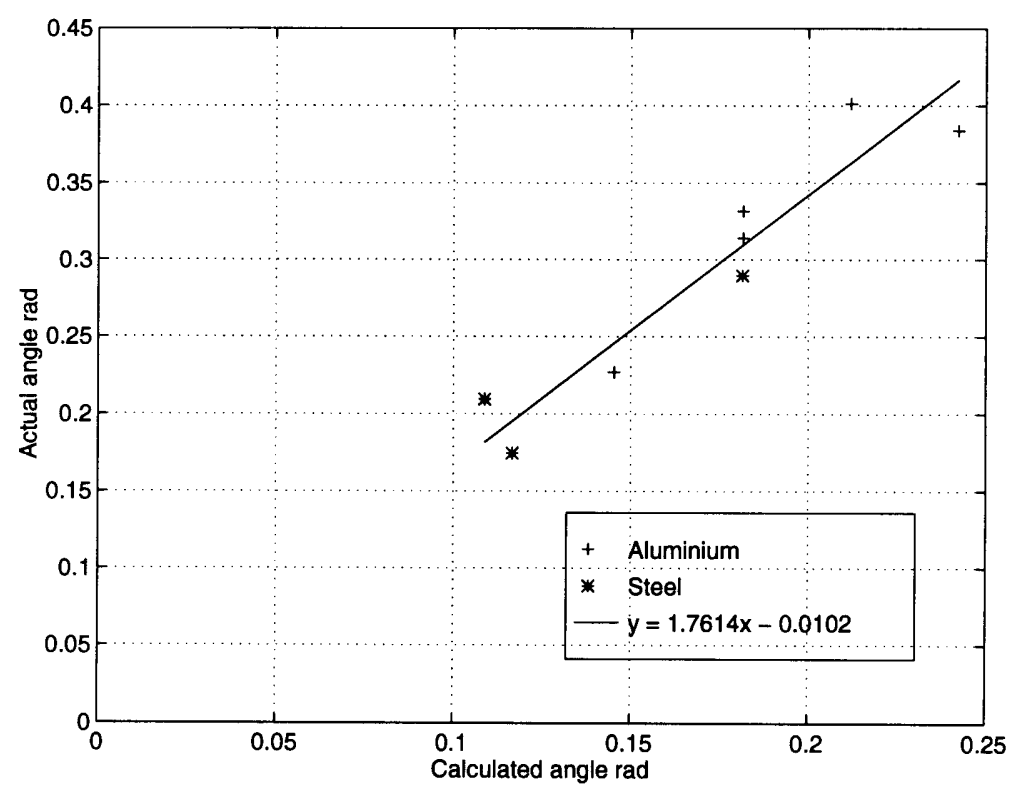

Figure 5-8: Comparison of cross spring angle

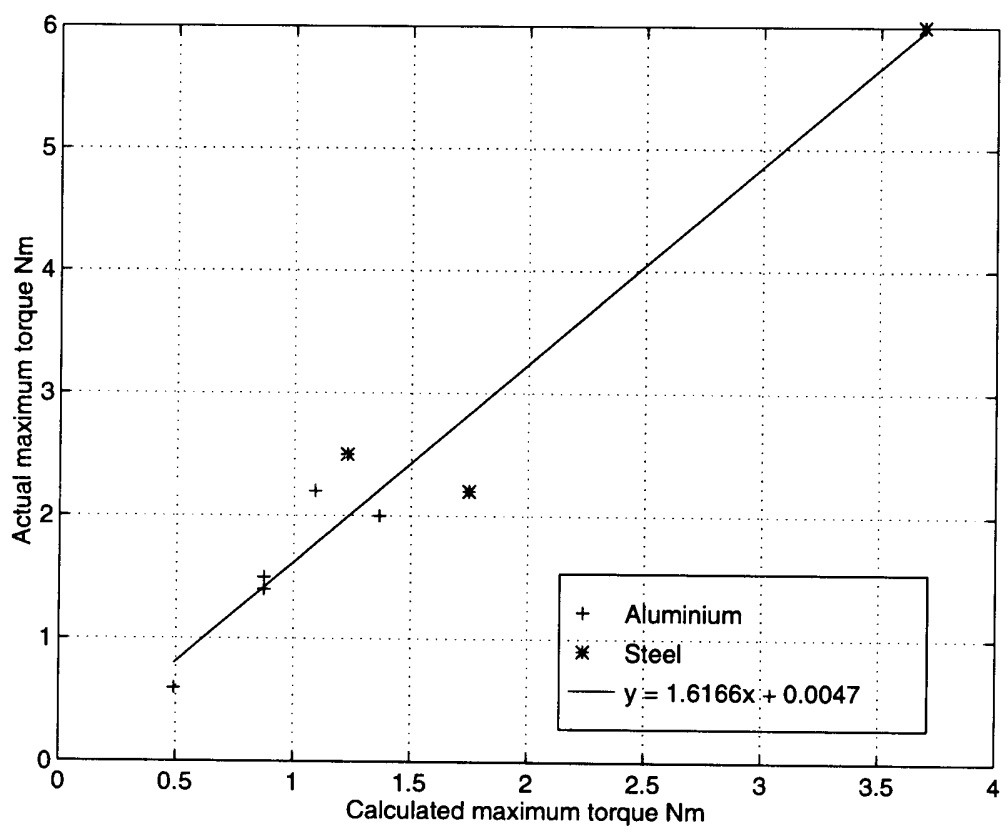

Figure 5-9: Comparison of cross spring torque 


\subsection{Actuator Spring Design}

The final spring chosen for the actuator had a web thickness of $0.05 "(1.3 \mathrm{~mm})$, the spring dimensions were $0.5 "(12.7 \mathrm{~mm})$ by $0.85 "(21.6 \mathrm{~mm})$, and the length was $2.4 "$ $(61 \mathrm{~mm})$.

Figure 5-10 shows the result of a test of the stiffness of this spring, using the encoder on the motor to measure the angle of twist. The kink in the middle is due to the backlash of the motor. The stiffness is slightly different in different directions of twist, being $46.3 \mathrm{Nm} / \mathrm{rad}$ in one direction and $45.0 \mathrm{Nm} / \mathrm{rad}$ in the other direction. In a different test the maximum angle of twist before yielding was found to be 7.4 degrees or $0.129 \mathrm{rad}$.

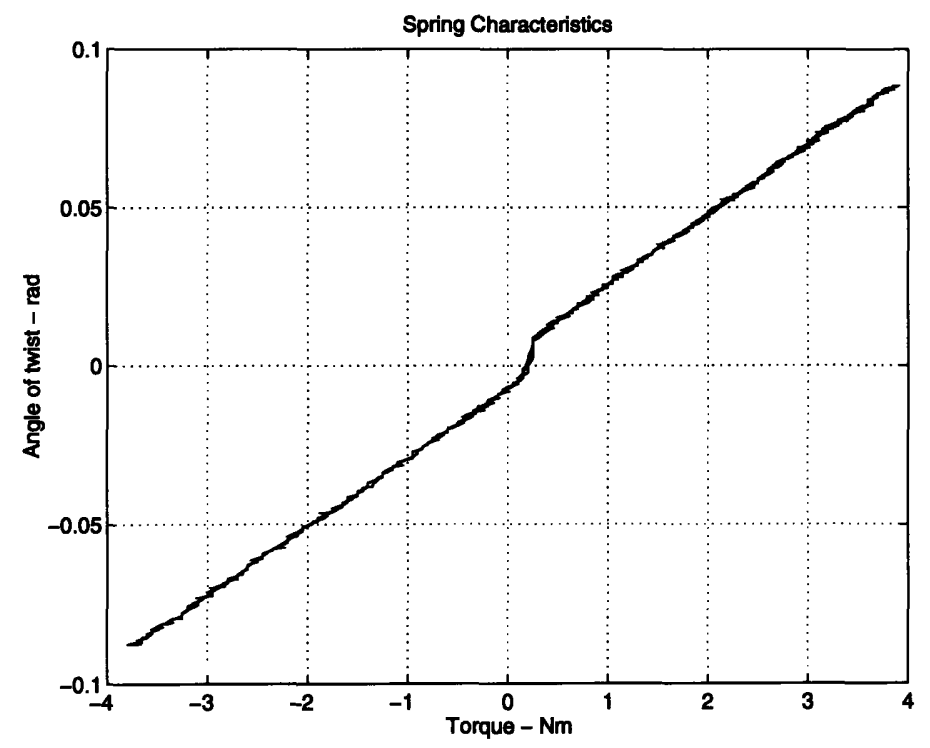

Figure 5-10: Plot of spring torque against spring angle

\subsection{Force sensor selection}

As well as providing elasticity the spring also acts as a force sensor, allowing the actuator output force to be controlled. As the spring is linear, a measurement of the angle of twist will also give the force. This measurement is difficult in this case since the angle is small (generally less than 5 degrees). The next two sections describe sensors which were used to measure the angle and the force.

\subsubsection{Sensor 1 : Potentiometer}

There are essentially two ways to measure the twist in the spring, firstly by measuring the angle of both ends and subtracting the results, and secondly to measure 
the angle directly. The latter method was chosen as it offered a less complex implementation requiring fewer sensors, less calibration, and potentially greater accuracy due to increased resolution. A potentiometer was used, the shaft being connected to the actuator output, and the body connected to the output of the motor with a stiff support. This is shown in Figure 5-11.

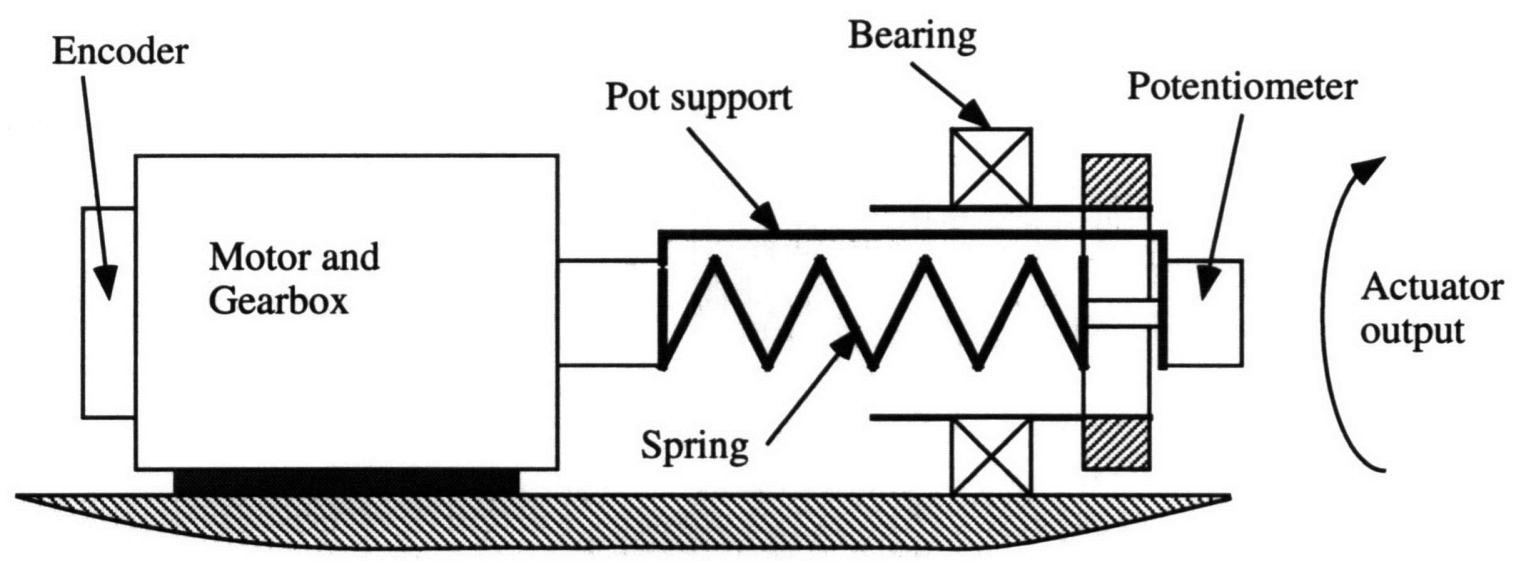

Figure 5-11: Schematic of potentiometer attachment

The potentiometer used was a $10 \mathrm{k} \Omega$ Spectrol Model 140 precision type. The signal from this was sampled by the control circuit (see section 5.7), and was also differentiated using an analogue circuit.

This sensor worked surprisingly well but had a number of problems. Firstly the signal was noisy (and so its differential was even more noisy) due to the action of the wiper moving over the resistive element. As the angle measured was small high gains were needed to amplify the signal, which also amplified the noise. Secondly it was felt that the steel strip that held the body of the pot might flex and twist as the angle changed, making the results inaccurate. The sensor was difficult to assemble, and had to be set up very carefully, all of which was lost when the system was disassembled. It was also bulky, which was undesirable from the mechanical design point of view. A one piece sensor was developed to overcome some of these problems, which is described in the following section.

\subsubsection{Sensor 2 : Strain Gauges}

This sensor measures the force by looking at the strain developed on the spring webs while it is twisted. It was assumed that the strain would vary linearly with the load, which was confirmed by experiment (discussed later in this section). The cross shaped spring described in the previous section, and illustrated in Figure 5-5 lent itself to instrumentation in this way, the gauges being easily mounted on the flats of the spring. A full bridge can be made by putting 2 gauges on opposite sides of the web, as illustrated in Figure 5-12. The gauges are aligned with the direction of 
maximum stress, which is at 45 degrees to the axis of twist for torsion. Figure 5-13 shows a closeup of the actuator and spring. The strain gauges are not quite visible, but are mounted just where the spring goes into the axle tube.

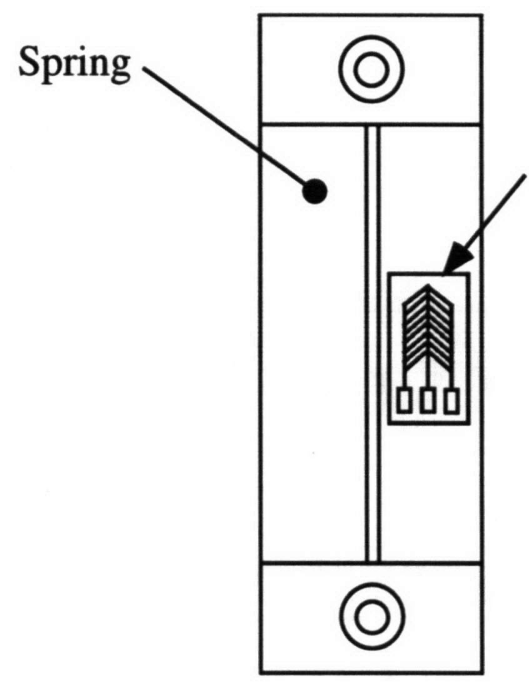

Front View

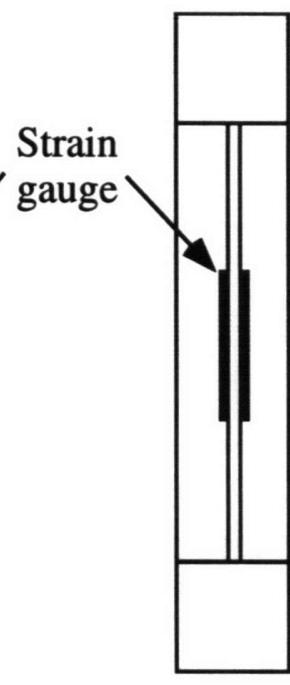

Side View

Figure 5-12: Showing position of gauges on the spring

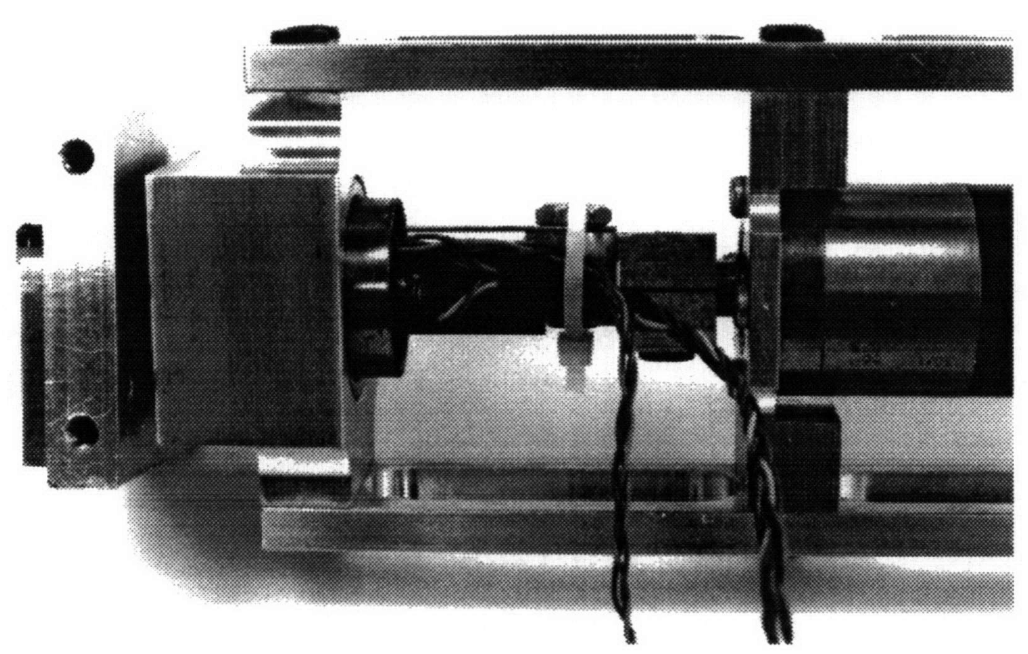

Figure 5-13: Closeup of spring

The gauges used were Micro Measurements CEA-06-062UV-350[33]. An Analog Devices 1B31AN Strain Gauge Conditioner was used to measure, amplify and filter the signal from the gauges, which was then differentiated and sampled for control purposes (see section 5.7). 
The signals from the strain gauges were considerably less noisy than the potentiometer signals (once they had been shielded correctly). The gain and offset of the amplifier is easily adjusted, so calibration of the sensor is easy. A plot of the relationship between the potentiometer and the strain gauge readings is included in Figure 5-14 below. The "staircase" like shape of this plot probably results from the movement of the pot shaft holder.

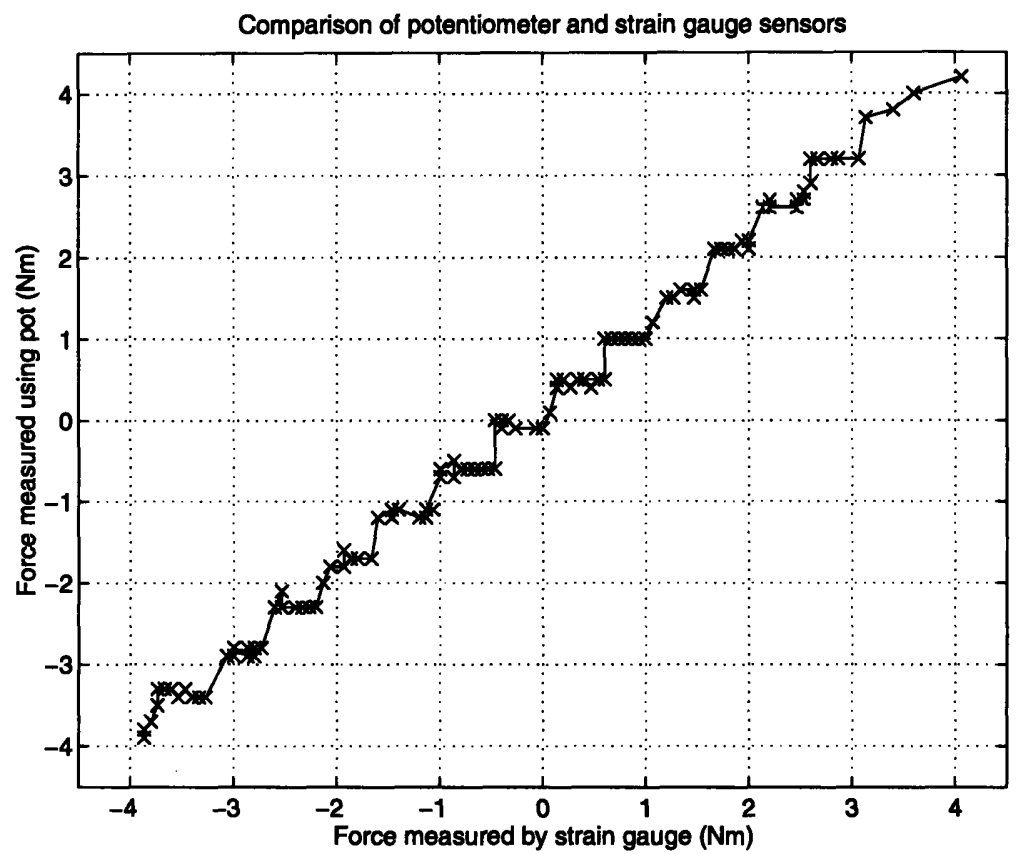

Figure 5-14: Plot of strain gauge reading versus potentiometer reading

Figure 5-15 shows a plot of the strain gauge reading versus actual torque, measured using a spring. The ratio between strain gauge reading and actual torque is 20 .

Strain gauges are notorious for being difficult to use in noisy environments (for example near large motors using $\mathrm{PWM}^{1}$ ). This problem is present in this application but is helped somewhat by the fact that the angle of twist, and so the strains being measured are large compared to normal strain gauge applications, so the gain of the amplifier can be small. On the other hand, the noise is increased when the signal is differentiated (see section 5.7). Careful shielding of the system reduced the noise to a tolerable level.

\footnotetext{
${ }^{1}$ Pulse Width Modulated (PWM) is a common technique for driving DC brushed electric motors
} 


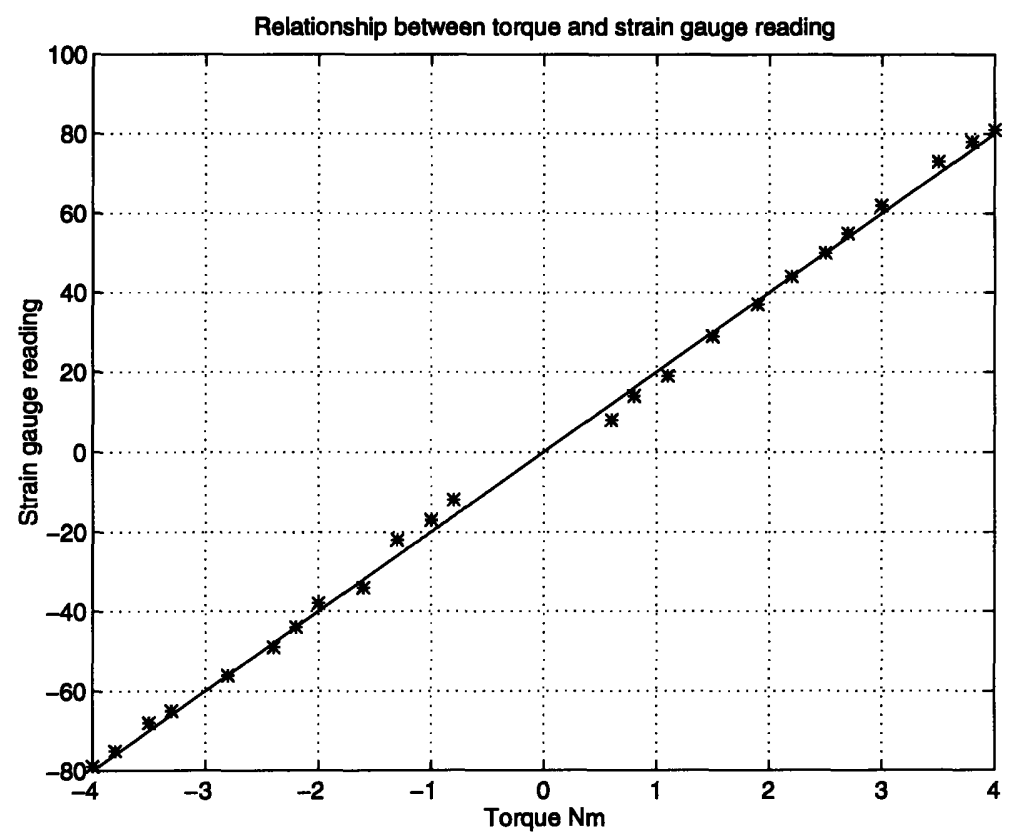

Figure 5-15: Plot of strain reading versus measured torque

\subsection{Electronic Setup}

This section describes the electronics and control hardware that was used to implement the torque control. A schematic of the whole system is shown in Figure 5-16. The strain gauges are connected to a sensor board, where their signal is amplified, filtered and differentiated, before being passed to the motor board. The cut off frequency of the strain gauge amplifier is $2 \mathrm{kHz}$, and of the differentiator $100 \mathrm{~Hz}$. These values were selected to ensure that there was not significant phase roll over the working frequency of the actuator (which is 0 to $20 \mathrm{~Hz}$ ).

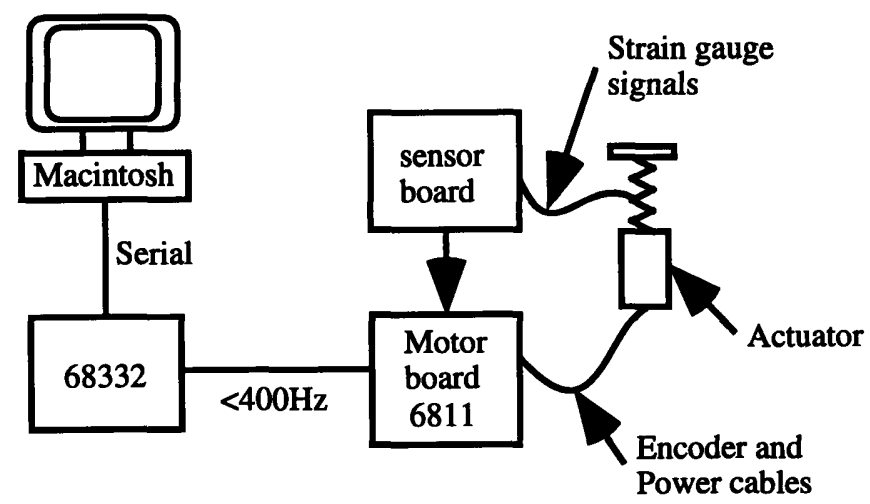

Figure 5-16: The complete system block diagram 
The motor board contains a Motorola 6811 micro-controller, on which the control code runs. The 6811 has 4 analogue to digital converters, which are used to sample the sensor readings. The control loop is set to run at $1 \mathrm{kHz}$. The board also contains hardware to read the encoder on the motor, a PWM generating circuit, and a $\mathrm{H}$ bridge to provide power to the motor. There is a differentially driven communication bus to the Motorola 68332 processor. Code for the 6811 is hand coded in assembly language, and then down-loaded from the serial line of the Macintosh PC. The motor board essentially contains all that is need to control the motor.

The 68332 is used for higher level processing. It communicates with the motor board at up to $400 \mathrm{~Hz}$, sending gains and set-points for the control loop, and receiving data. The processor runs L, a subset of Common Lisp, written by Rodney Brooks at MIT [6]. The front end of the 68332 is a Macintosh PC, where the data from the actuator can be displayed and analysed. 


\subsection{Control}

The control loop implemented on the 6811 is similar to that detailed in Chapter 3. A block diagram showing all the systems is included in Figure 5-17. There are essentially five parts in the diagram, the sensor differentiation part, the PID servo on desired torque, the feedforward terms for desired torque and acceleration of the output shaft, and the feedforward model of the motor.

Some comments about the block diagram:

- The A/D converter on the 6811 is only 8 bits, so in order to reduce the errors from differentiating small signals in software, the analogue sensor readings were differentiated using an analogue circuit. The encoder reading is 16 bits, and changes enough in normal use to allow differentiation in software. To help this, the effective sampling frequency for the encoder was reduced to $250 \mathrm{~Hz}$.

- The feedforward model described in section 3.3 was implemented apart from the term which included the second differential of the desired force. This was because of the difficulties of differentiating small fixed numbers in software. The encoder velocity was calculated by differencing the encoder readings. This was used with the differentiated strain gauge signal to calculate the acceleration of the output shaft $\ddot{\theta}_{l}$. The parameter "Fgain" is a gain parameter corresponding to the amount of feedforward used ( $K_{f f}$ in figure 3-5).

- The readings for the strain gauges were converted to 16 bits for the servo loop calculations, and 16 bit gains were used. The output of the servo loop is a desired current, an 8 bit number. This is the input to the feedforward motor model, which produces a PWM duty cycle command ( 8 bits) and a direction (1 bit) which are applied to the motor. The 6811 carries out fixed point arithmetic, which is why there are so many "divide by 256 " terms in the block diagram.

- The motor is driven by an $\mathrm{H}$-bridge, using $\mathrm{PWM}$ at $48 \mathrm{~V}$, and $31.25 \mathrm{kHz}$. A simple feedforward model of the motor was used to calculate the correct voltage to apply to ensure that the motor current (and so the torque) followed that commanded by the control system. The 6811 was not fast enough to run a separate current feedback loop as well as the torque control. A full feedforward model would include terms to compensate for the motor resistance, inductance and back emf (see Figure 5-18), however only terms for the resistance and the back emf were implemented. 


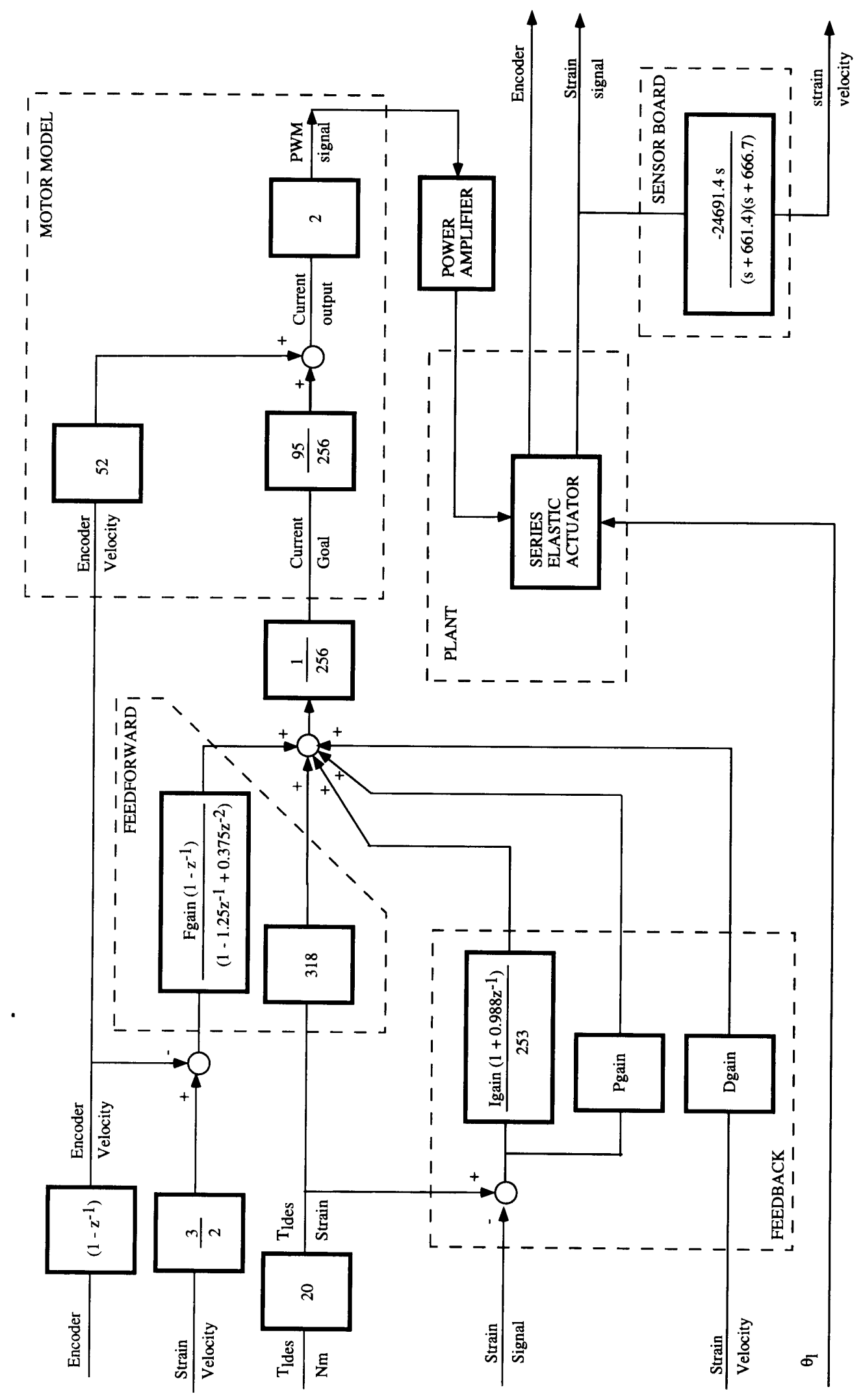

Figure 5-17: The control system 


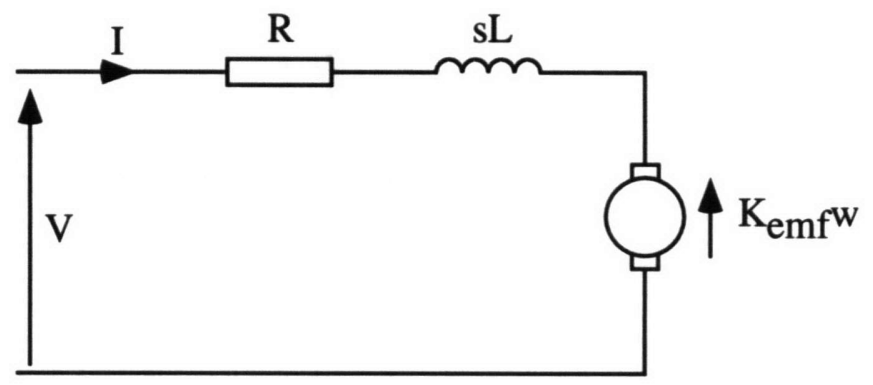

Figure 5-18: Motor model

\subsection{Test Rig}

As described in Chapter 4, a good test of the actuators performance is a measure of the range of output impedances that it can produce. To do this, a test rig was devised that allowed the position of the actuator shaft to be moved in a controlled fashion, independently of the torque output of the actuator. A photograph of the setup is shown in Figure 5-192. The torque controlled actuator is on the right, and the position controlled one on the left. It consists of the usual torque controlled actuator, with the end being driven by a RH-14-6002 $(24 \mathrm{~V}, 20 \mathrm{~W})$ motor, with a harmonic drive gearbox. This was chosen mainly because it was available, and the gearbox had minimal backlash. The control setup for this motor is identical to that of the torque controlled one, except that it servos to a position measured by its encoder.

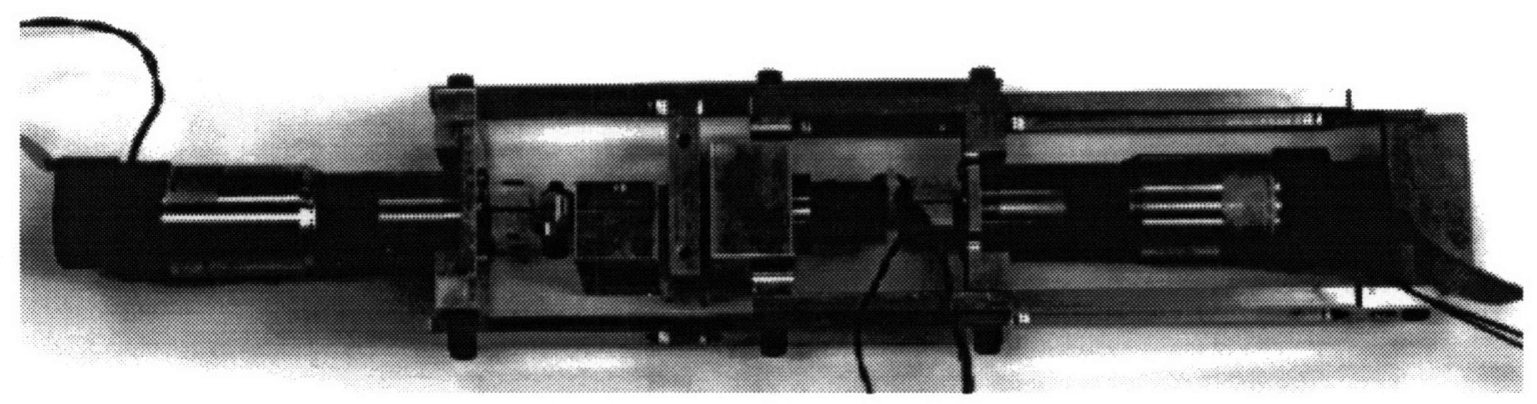

Figure 5-19: Photograph of the Actuator test rig

\footnotetext{
${ }^{2}$ The position controlled actuator illustrated is not the one referred to in the text, although the setup is the same
} 


\section{Chapter 6}

\section{Experimental Results}

\subsection{Introduction}

This chapter describes the experimental data taken from the series elastic actuator described in chapter 5 . The identification of the system is described, and the measured frequency response of both open and closed loop systems are included.

The calculations made to determine the frequency responses assume that the system is linear, while the actual system is affected to some extent by non-linearities such as friction, backlash and saturation. The plots are included as they represent how the system behaves in a compact format. An attempt was made to collect data in regions where the non-linear effects were not large, to improve accuracy.

The overall performance limits of the system were tested on a frequency by frequency, amplitude by amplitude basis, so as to capture the non-linear effects. Comparisons are drawn between the theoretical predictions and the actual experimental data.

\subsection{System Identification}

The system was identified using a sweep sine technique. Time domain data of the actuators behaviour was analysed using a simple single frequency Fourier transform, to capture the magnitudes and phases of the relevant signals. The esoteric nature of the control hardware used for the actuator made the use of a commercially available spectrum analyser difficult. The major drawback of the homemade system was that some of the calculations were carried out in fixed point arithmetic, causing rounding errors, especially when the system was trying to measure small signals.

The transfer functions between the motor current and the output force with the actuator output shaft clamped, and also between the output force and the motion of the output shaft were identified. The latter function is the output impedance of the actuator. Matlab was then used to fit a simple transfer function model to the experimental data. Figures 6-1 and 6-2 show the experimental results together with 
the fitted model responses.
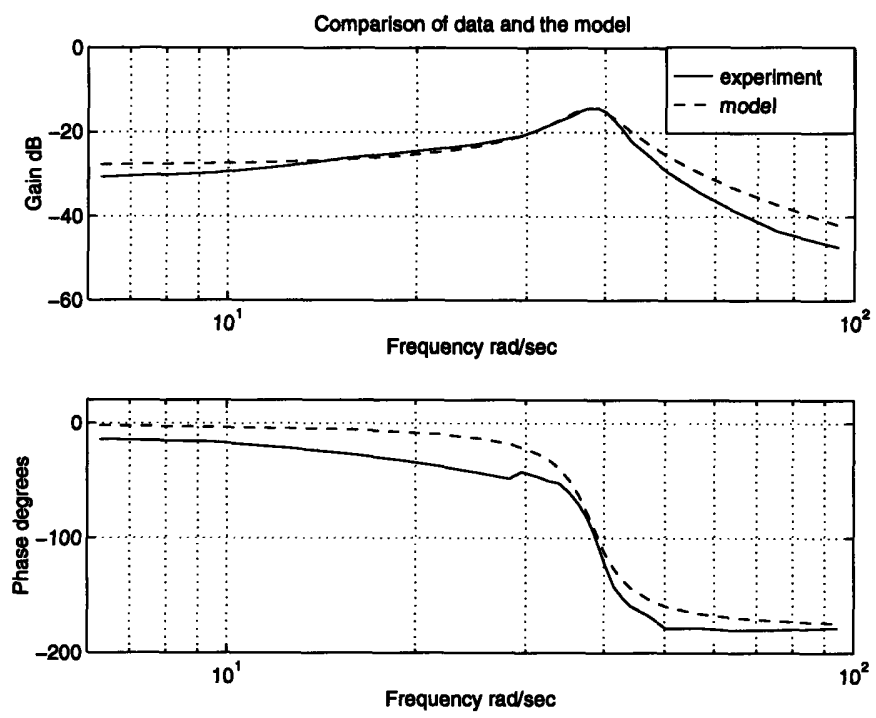

Figure 6-1: Bode plot for force transfer function
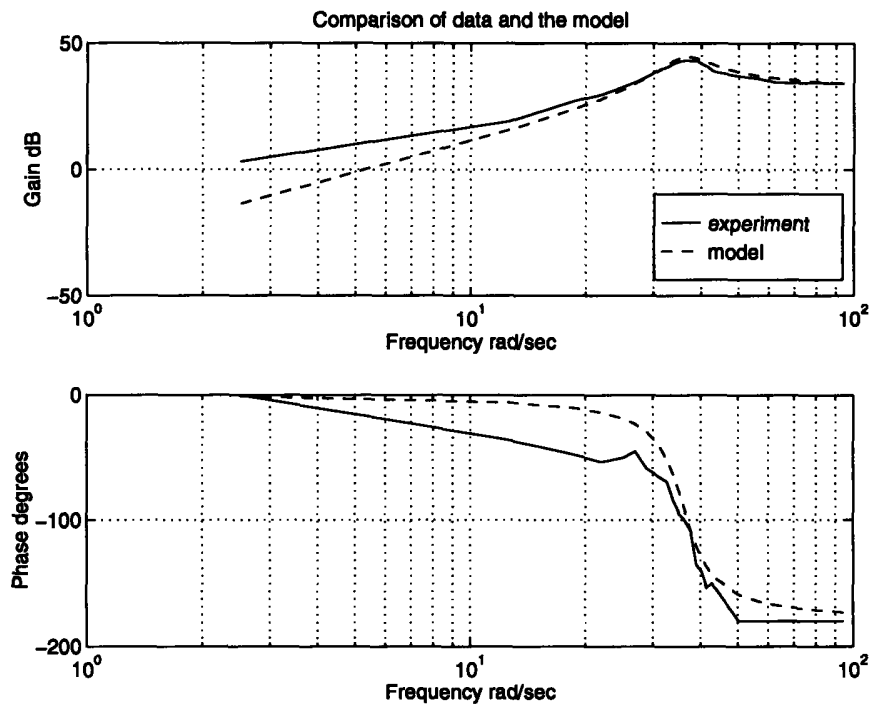

Figure 6-2: Bode plot for impedance transfer function

The data for the force transfer function shows a lower gain than expected at low frequencies as well as a significant phase lag, which is probably attributable to the effects of stiction in the bearings. It was noticed that the sine waveforms for current were actually clipped at these frequencies. The shape of the bode plot is characteristic 
of a lightly damped second order system. The identified model is given below.

$$
\frac{T_{l}}{T_{m}}(s)=\frac{59}{s^{2}+7.89 s+1475}
$$

The poles are at $-3.945 \pm 38.2026 j$, they have natural frequency of $38.406 \mathrm{rad} / \mathrm{sec}$, and damping factor $\zeta=0.1027$. The theoretical transfer function is rewritten below

$$
\frac{T_{l}}{T_{m}}(s)=\frac{1}{1+s^{2} J_{m} / k_{s}}=\frac{k_{s} / J_{m}}{s^{2}+k_{s} / J_{m}}
$$

The quoted value for the motor inertia is $0.02 \mathrm{Kgm}^{2}$, and the measured value of the spring constant is $46 \mathrm{Nm} / \mathrm{rad}$ (see chapter 5), which corresponds to a natural frequency of $48.0 \mathrm{rad} / \mathrm{sec}$. The measured natural frequency corresponds to a motor inertia of $0.03 \mathrm{Kgm}^{2}$, which is higher than the quoted value of $0.02 \mathrm{Kgm}^{2}$. This is not surprising since the quoted inertia does not include the inertias of the gearbox and the spring. The denominator of the identified transfer function above (59) does not compare well to the value for $k_{s} / J_{m}$ which is 1475 . This is because the transfer function related motor current rather than motor force to output force. Once the correct scaling factor is used the denominator becomes 1494, which is close to 1475 .

The results of the impedance identification (Figure 6-2), also show a second order system with under-damped poles. The identified transfer function is given by

$$
\frac{T_{l}}{\theta_{l}}(s)=\frac{-44 s^{2}}{s^{2}+9.25 s+1306}
$$

compared to the predicted transfer function

$$
\frac{T_{l}}{\theta_{l}}(s)=\frac{-s^{2} J_{m}}{1+s^{2} J_{m} / k_{s}}=\frac{-s^{2} k_{s}}{s^{2}+k_{s} / J_{m}}
$$

The poles of the identified system are at $-4.625 \pm 35.84 j$, at $36.14 \mathrm{rad} / \mathrm{sec}$ and with a damping factor of 0.1280 . This is slightly different than above, which is probably due to experimental error, and could also be due to frictional effects in the bearings which are more noticeable when the output shaft is moving. The denominator of the transfer function $\left(-44 s^{2}\right)$ implies that the spring constant is $44 \mathrm{Nm} / \mathrm{rad}$ which compares favourably with the measured value of $46 \mathrm{Nm} / \mathrm{rad}$.

The identified transfer functions give useful information about the system, and will be used to tune the control loop. 


\subsection{Feedback Tuning and Performance - Theoret- ical}

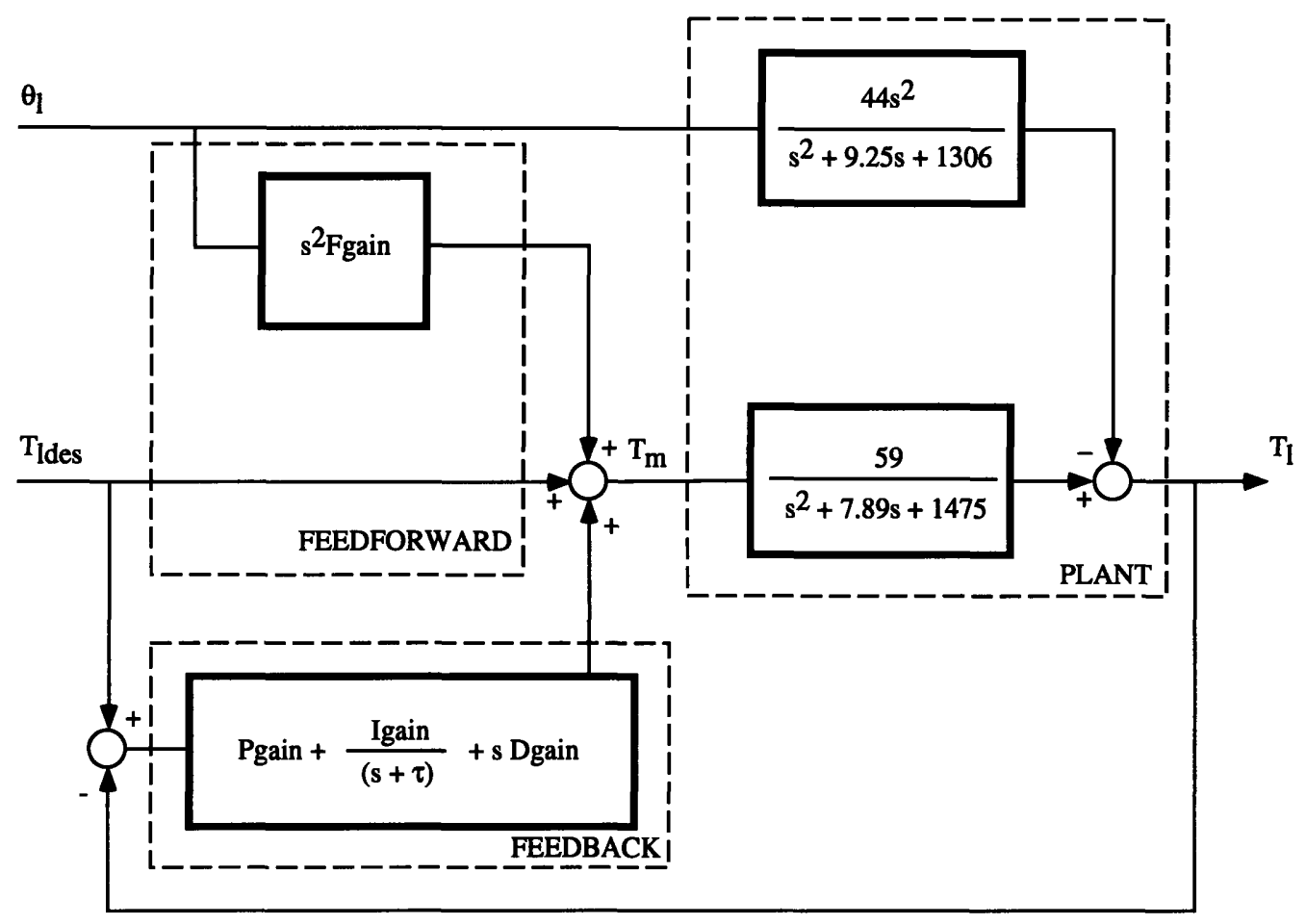

Figure 6-3: The closed loop system

The full closed loop system is illustrated in Figure 5-17, a more condensed version being included in figure 6-3. As discussed previously (sections 3.4 and 5.7), the control action consists of two feedforward terms and a PID servo on the desired torque. Root locus techniques were used to select appropriate values for the gains in the system, making the system performance fast, with reasonable damping, while ensuring that the gains were not set so high as to make the noise in the system intolerable. The $K_{f f}$ gain was selected by trial and error as described in section 6.5 . The gains and the position of the integral gain pole are given in table 6.1 below.

\begin{tabular}{||l|r||}
\hline Gain & Value \\
\hline Pgain & 1908 \\
\hline Igain & 977 \\
\hline Vgain & 362 \\
\hline$\tau$ & 11.86 \\
\hline
\end{tabular}

Table 6.1: Gains for closed loop system 


\begin{tabular}{||r|r|r||}
\hline \multicolumn{1}{|c|}{ Pole position } & Damping & Frequency $(\mathrm{rad} / \mathrm{sec})$ \\
\hline$-3.8450+38.2128 \mathrm{i}$ & 0.1001 & 38.4057 \\
\hline$-3.8450-38.2128 \mathrm{i}$ & 0.1001 & 38.4057 \\
\hline$-65.7259+75.5188 \mathrm{i}$ & 0.6565 & 100.1148 \\
\hline$-65.7259+75.5188 \mathrm{i}$ & 0.6565 & 100.1148 \\
\hline-13.9036 & 1.0000 & 13.9036 \\
\hline
\end{tabular}

Table 6.2: Closed loop poles

Table 6.2 shows the expected location of the closed loop poles of the system when these gains are applied. Figures 6-4 and 6-5 show the effect of closing the feedback loop, the natural frequency of the system changing to about $38 \mathrm{rad} / \mathrm{sec}$, and the size of the resonant peak being reduced. The impedance of the output is also lower at low frequencies, and remaining the same at high frequencies.
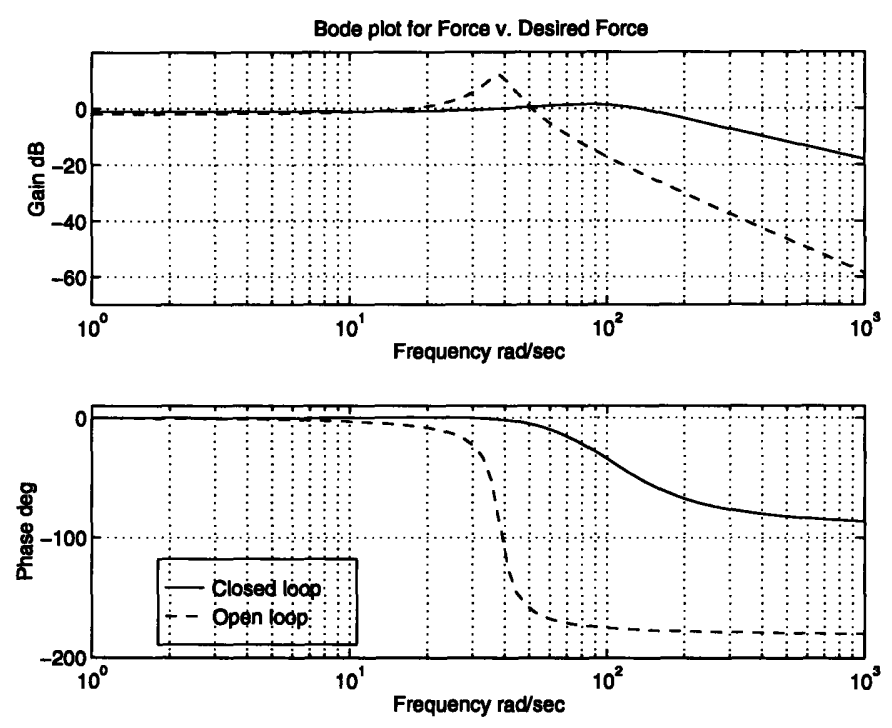

Figure 6-4: Bode plot for simulated closed loop force transfer function

\subsection{Feedback Tuning and Performance - Experi- mental}

The gains in Table 6.1 were used in the control system for the actuator. To check the performance of the closed loop, the system was identified again, using sweep sine as before. The results of this test are shown in Figure 6-6. This plot shows the open loop, the predicted closed loop and the actual closed loop response. The closed loop response is better than the open loop, and the pole due to the resonance of the motor 

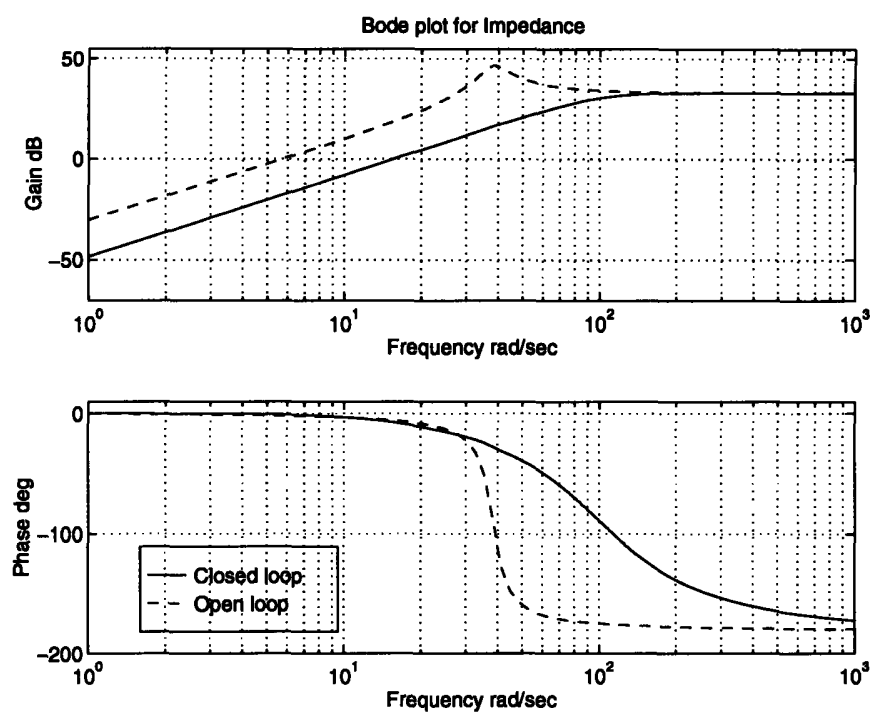

Figure 6-5: Bode plot for simulated closed loop impedance transfer function

mass on the spring has been effectively relocated to a higher frequency.

However, the actual response is quite different from that predicted by the theory. The response has a higher gain than predicted at low frequencies, and there is what appears to be an under-damped pole at $63 \mathrm{rad} / \mathrm{sec}$. The phase rolls off towards -180 degrees which is also unexpected, although the approximate gradient of the phase roll is the same as that predicted by the simulation. To determine whether this extra "pole" was caused by non-linear effects such as backlash or saturation, the test was conducted again, with a bias on the desired torque. It was difficult to completely eliminate the backlash by this method, but its effect was reduced significantly over all frequencies, especially so for low frequencies. Small torques had to be used to obtain this, which made the calculation less accurate due to rounding errors, but a general trend can be seen in Figure 6-7. The biased runs follow the simulated closed loop behaviour much more closely than the run where the backlash was significant. The peak in their bode plots also seems to fall near where the predicted maximum should be. The phase plot is also interesting, as the jump in phase is reduced in size when the backlash is reduced. The gradient of the phase roll is similar to that predicted by the simulation. All of this suggests that the backlash is a contributory factor in the "pole" at $63 \mathrm{rad} / \mathrm{sec}$.

The backlash is like a dead band where the motor mass can accelerate without contacting the spring. It is possible that this non-linearity can lead to oscillation (see Khalil [20]), with a precise resonant frequency, dependent on the size of the nonlinearity, and the characteristics of the rest of the system. This would also explain why this effect is only observed when the feedback loop is closed.

Figure 6-8 shows the results for the closed loop impedance. Again the closed loop system is considerably better than the open loop, the impedance being reduced for 

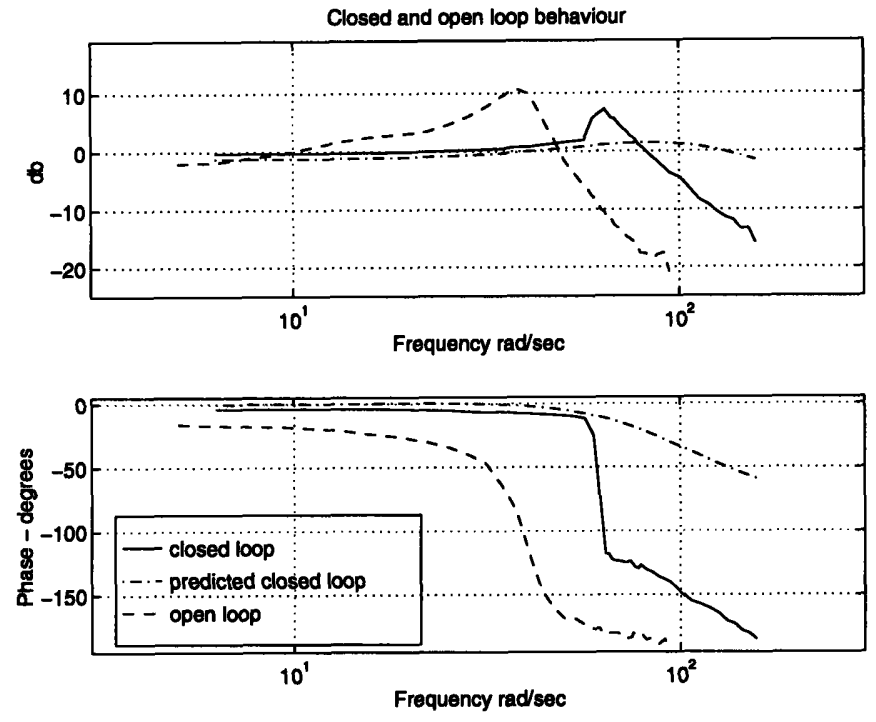

Figure 6-6: Bode plot for identified closed loop force transfer function
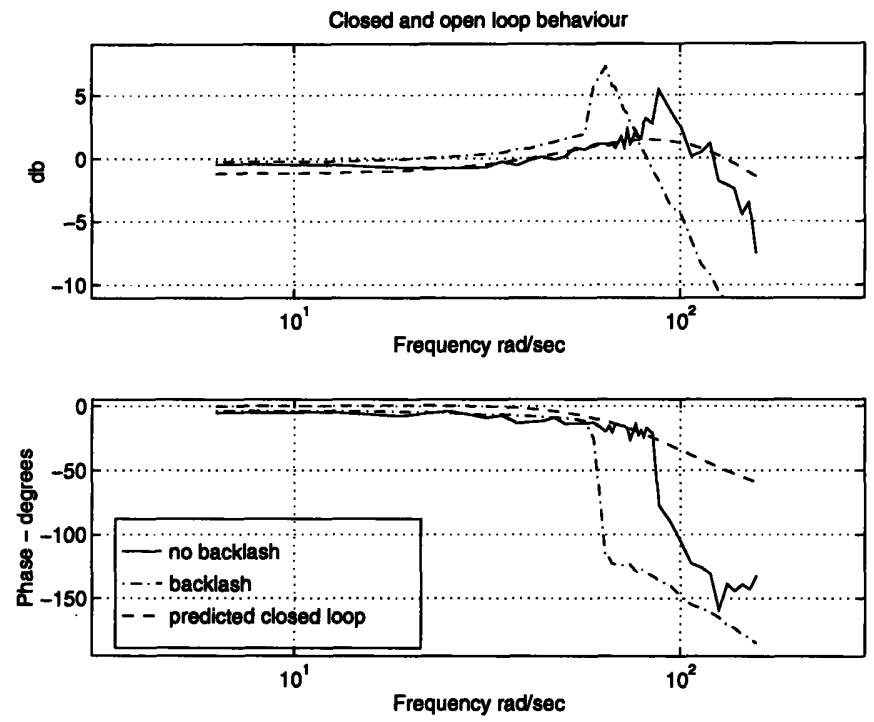

Figure 6-7: Bode plot of the dependence of the system closed loop response of backlash 

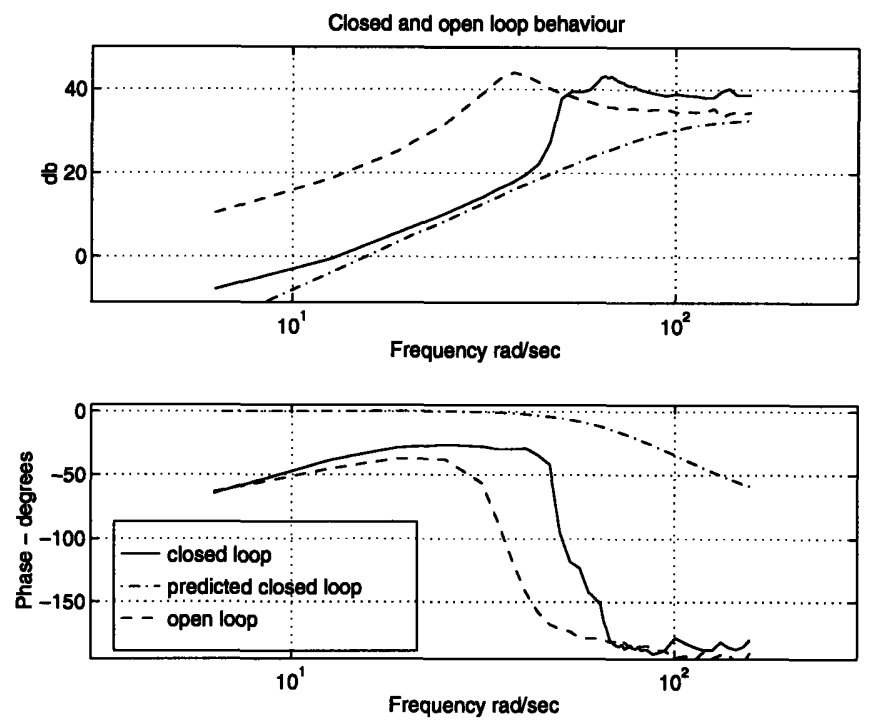

Figure 6-8: Bode plot for identified closed loop impedance transfer function

all frequencies less than about $60 \mathrm{rad} / \mathrm{sec}$. It should be noted that the method used to obtain the magnitudes and phases is less accurate for small gains, so the phase plots for small frequencies are inaccurate. Again the actual response but is not very similar to that predicted by the theory. The under-damped response is clear here, although it happens at a lower frequency (about $8 \mathrm{~Hz}$ or $50 \mathrm{rad} / \mathrm{sec}$ ). It was hoped to eliminate the effects of backlash for this test, however the position motion required to ensure that the motors force always remained positive was so small that no useful data could be collected. The high frequency impedance corresponds to a spring constant to of about $58 \mathrm{Nm} / \mathrm{rad}$.

\subsection{Assessing the effect of the $K_{f f}$ gain}

From looking at the control system in figure 6-3, The open loop transfer function between force output and the motion of the output shaft including the feedforward term is given by

$$
\frac{T_{l}}{\theta_{l}}(s)=\frac{-s^{2} J_{m}\left(1-K_{f f}\right)}{1+s^{2} J_{m} / k_{s}}=\frac{-s^{2} k_{s}\left(1-K_{f f}\right)}{s^{2}+k_{s} / J_{m}}
$$

$K_{f f}$ reduces the effective stiffness of the spring to $k_{s}\left(1-K_{f f}\right)$. It is important that $K_{f f}$ is less than one otherwise non passive behaviour will result. To determine the effect on the real system of changing $K_{f f}$, the impedance of the system was measured as it was varied. The results for both the open loop and closed loop cases are illustrated in Figure 6-9. 
It is clear from the figure that increasing $K_{f f}$ has the desired effect of reducing the output impedance at low frequencies. At high frequencies the system is dominated by the non-linear effects of saturation and backlash, so the gain has little obvious effect. The signal is noisy, and a tradeoff has to be made between the size of the gain and the actual overall system performance. For gains above $K_{f f}=0.31$ the overall system performance is degraded. If the gain is raised too much the system behaviour changes, as can be seen in the case where $K_{f f}=0.54$. The value $K_{f f}=0.27$ was selected as giving the best tradeoff between performance and noise.
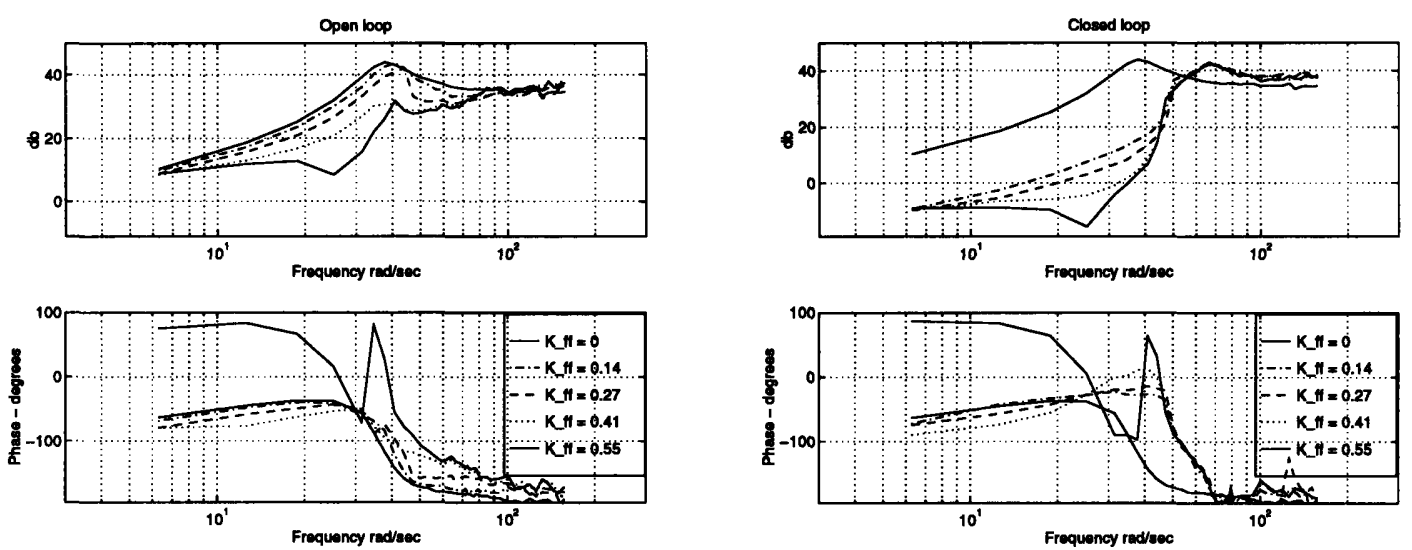

Figure 6-9: Bode plot showing effect of $K_{f f}$

\subsection{System Performance}

Figure 6-10 shows the bode plots for the system performance with the final gains and feedforward terms. Both the open and closed loop responses are included.
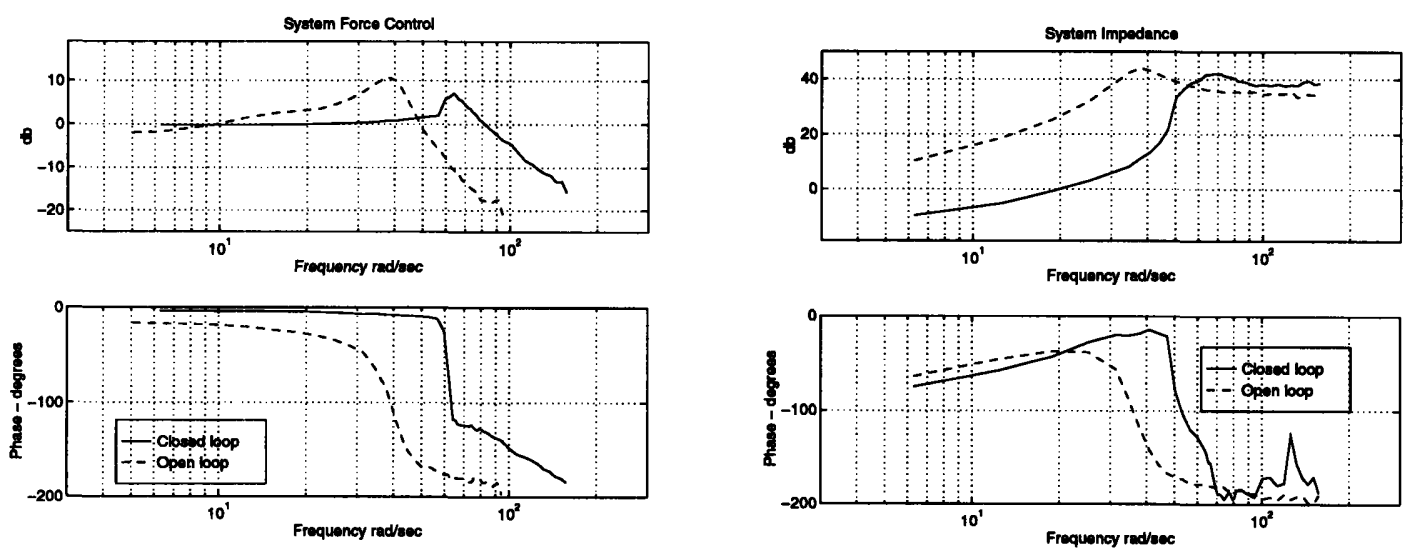

Figure 6-10: Bode plot showing performance of system 
The system torque control performance while in contact with a hard (Aluminium) and a sort (rubber) surface was also measured. Figure 6-11 shows the response to a square wave in commanded torque. The response for the soft surface is slightly less damped than for the hard one, but neither exhibits the instability common in this type of test (see An [2]). Figure 6-12 shows a similar test where the torque is commanded to follow a sine wave command. Again the performance is good in both cases. Figure 6-13 shows the force control performance at following a sine wave command when the output shaft of the actuator is also being moved. Performance in this kind of test is covered in more detail in the following section.
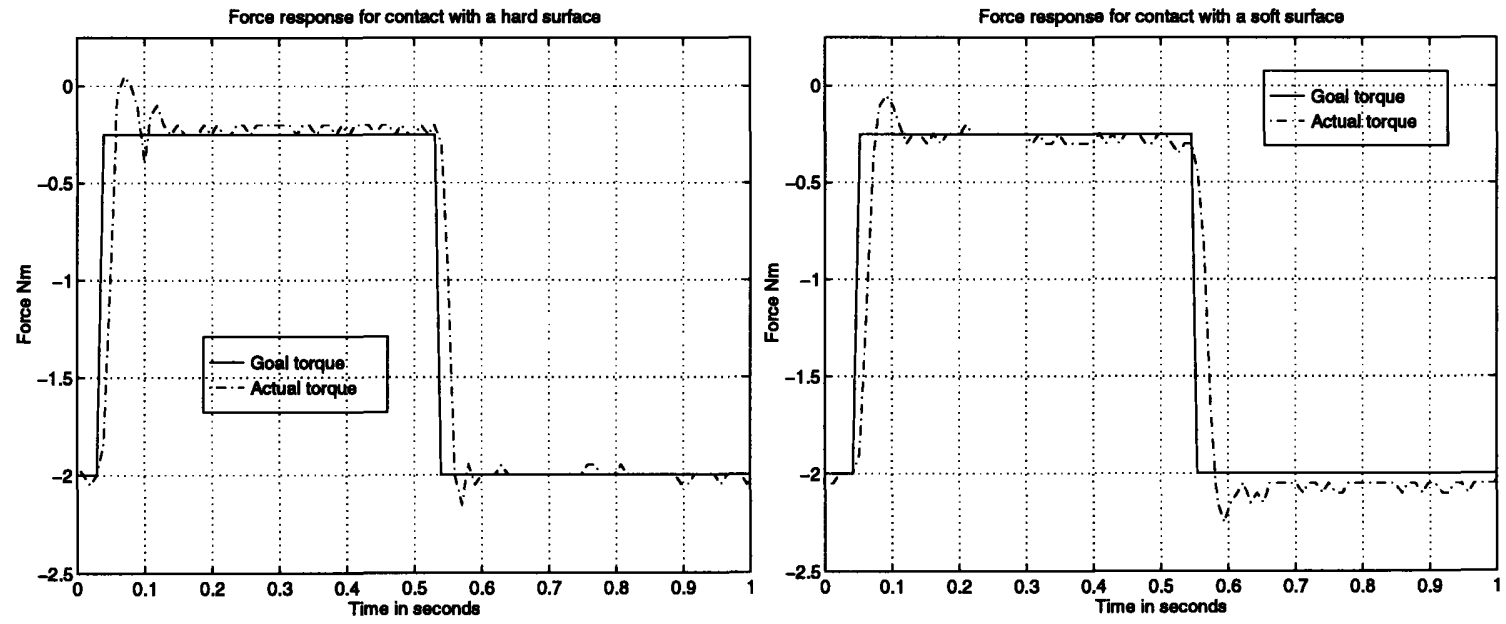

Figure 6-11: Square wave response
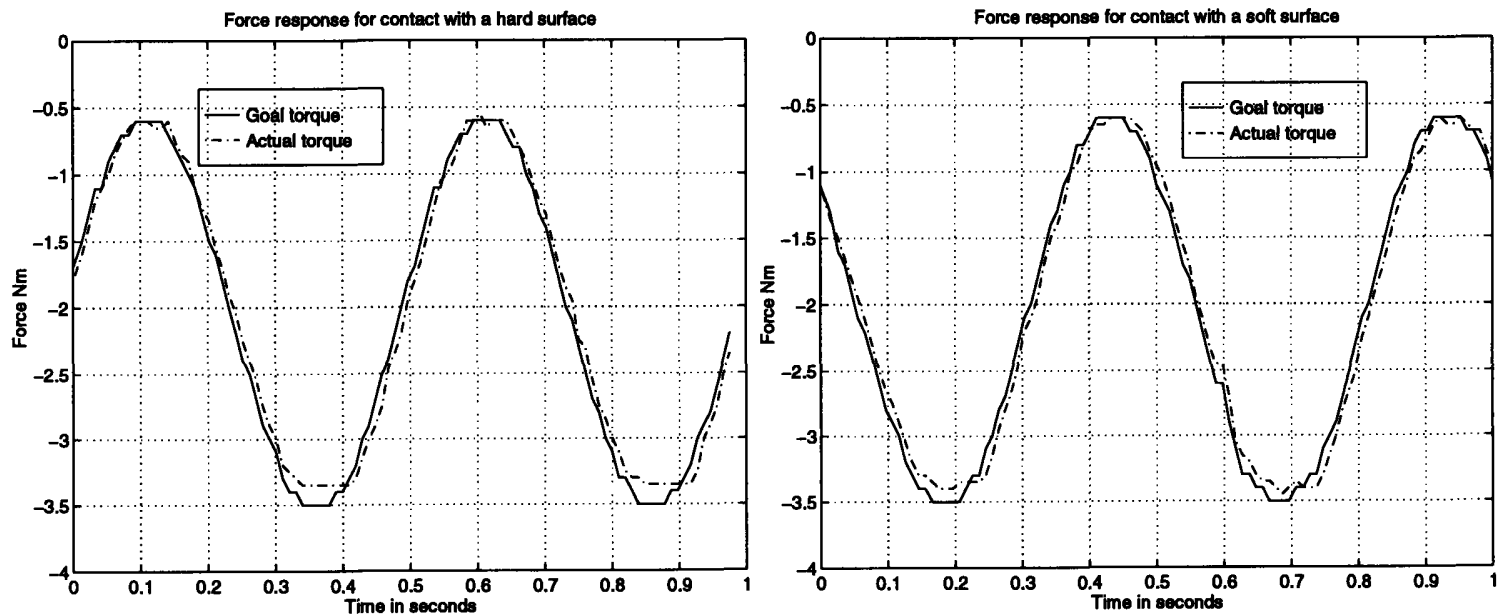

Figure 6-12: Sine wave response 

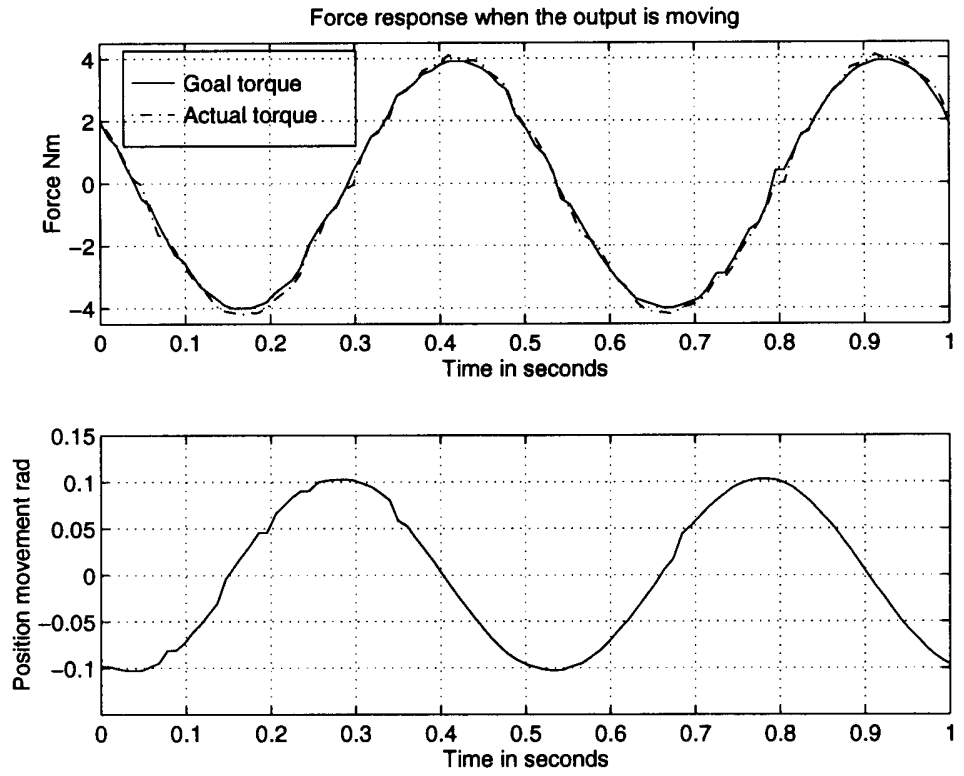

Figure 6-13: Torque control performance when the output shaft is moving

\subsection{Performance Limits}

The final series of tests which were carried out were to evaluate the performance of the actuator over a range of frequencies while attempting to provide force at a defined impedance. This can be directly compared to the results from chapter 4 .

The actuator was commanded to follow a sinusoidal force waveform, while its output shaft was moved at the same frequency, with a constant phase difference between the force command and the position waveform, so defining the impedance output of the system. The sizes and phases of the signals were varied to test over a range of impedances. Figure 6-13 shows a sample test condition. The error in the force tracking was measured and used to determine the maximum force that could be tracked with a defined error, at a certain impedance. The tester assumed that the error increased with the magnitude of the commanded force, and ramped the force up and down accordingly. Due to rounding errors the error was large for both large and small forces, with a minimum condition in between. The algorithm was chosen so that it always selected the maximum force for a given error tolerance.

Due to the limitations of the position controlled motor, the magnitude and phase of the motion were not always as specified for the test point. This made the actual impedance tested different from the desired impedance test-point. The same Fourier transform that was used to calculate the frequency responses (see section 6.2) was used to determine the exact impedance of the test-point, and then Matlab's interpolation routines were then used to convert the irregularly spaced data points onto a square grid so that they could be plotted.

Figure 6-14 shows the maximum force at $10 \%$ error for $2 \mathrm{~Hz}$, or $12 \mathrm{rad} / \mathrm{sec}$, together 
with a plot of the theoretical results. The actuator performance is good and is similar to that predicted by the saturation model.
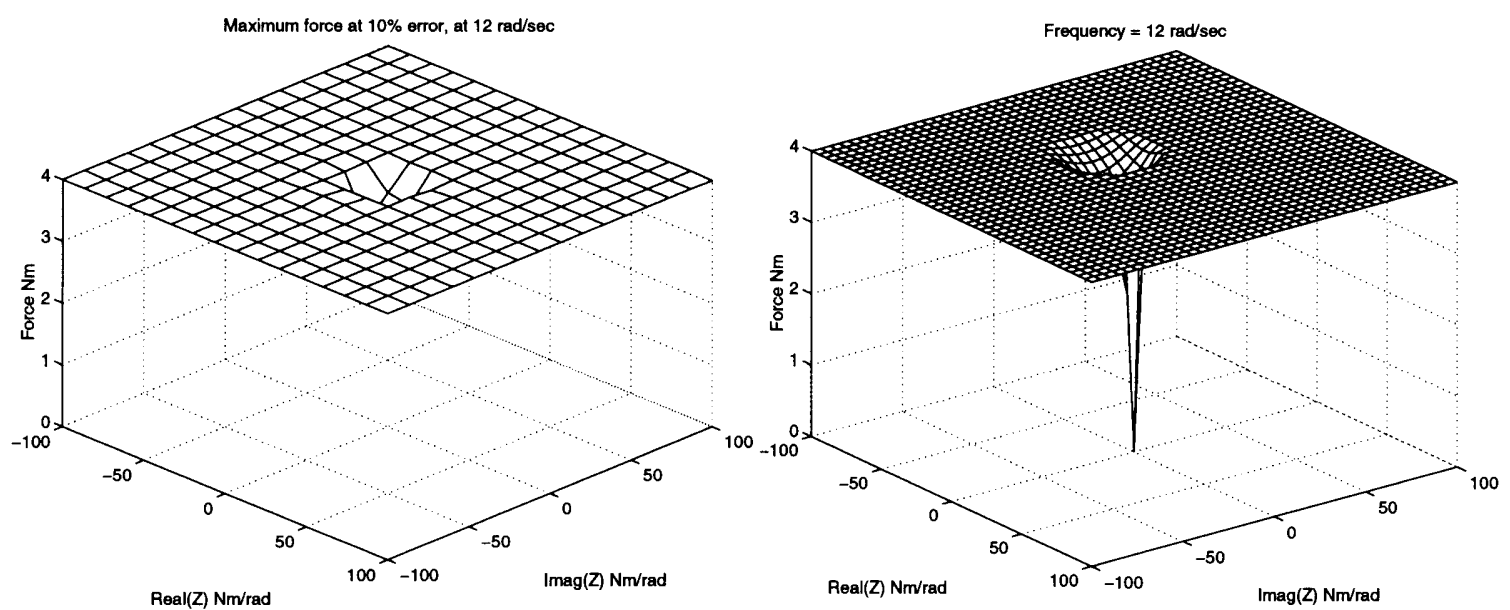

Figure 6-14: Performance results for $12 \mathrm{rad} / \mathrm{sec}$

When the frequency is increased to $4 \mathrm{~Hz}$, or $25 \mathrm{rad} / \mathrm{sec}$ (Figure 6-15), then there is a larger range of impedances where the force control does not work so well. The experimental results show an area where the output force is zero. This means that the tester could not find a force with $10 \%$ error at that impedance, not that the actuator outputs no force! The size of the hole is bigger than expected, which is probably due to inefficiencies in the motor, backlash friction, and a whole host of other non-linear effects. It is not clear why the surface is uneven.
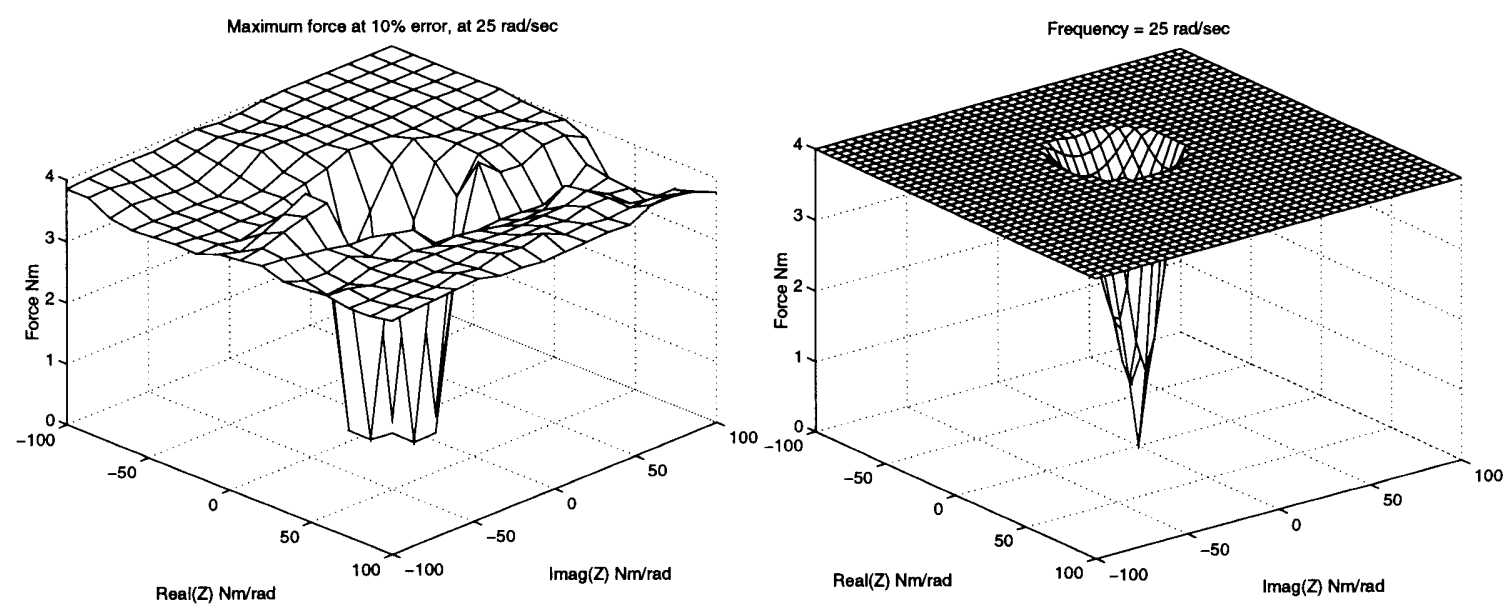

Figure 6-15: Performance results for $25 \mathrm{rad} / \mathrm{sec}$ 
The plot for $6 \mathrm{~Hz}(37.7 \mathrm{rad} / \mathrm{sec})$, which roughly corresponds to the natural frequency of the system is shown in Figure 6-16. The general shape of the two plots is similar - the actuator being unable to provide forces near zero impedance. The theory suggests that the actuator can output it's maximum force at large, and the experimental results do bear this out, although the magnitudes are lower in some places (about $3 \mathrm{Nm}$ as opposed to the maximum $4 \mathrm{Nm}$ ). The forces are lower for impedances with a positive real part, (mass-like behaviour), which is a trend which continues as the frequency is increased. The size of the hole is larger than predicted by the theory.
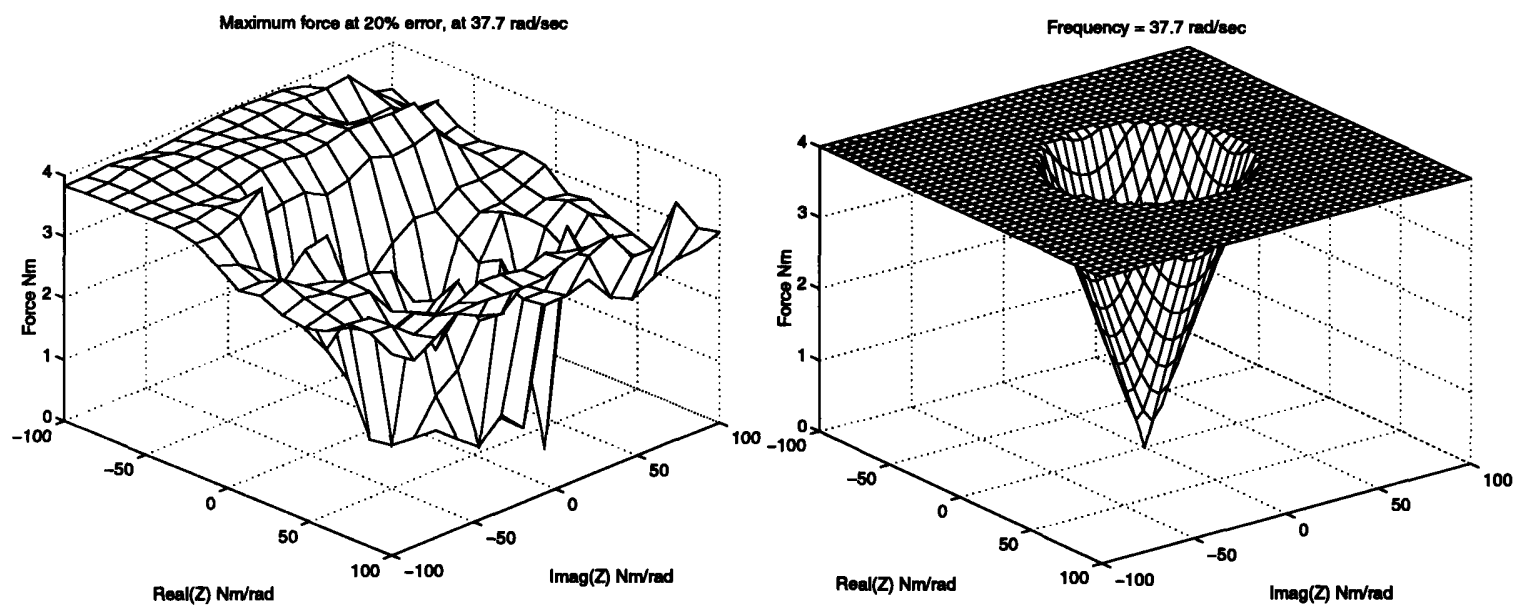

Figure 6-16: Performance results for $37.7 \mathrm{rad} / \mathrm{sec}$
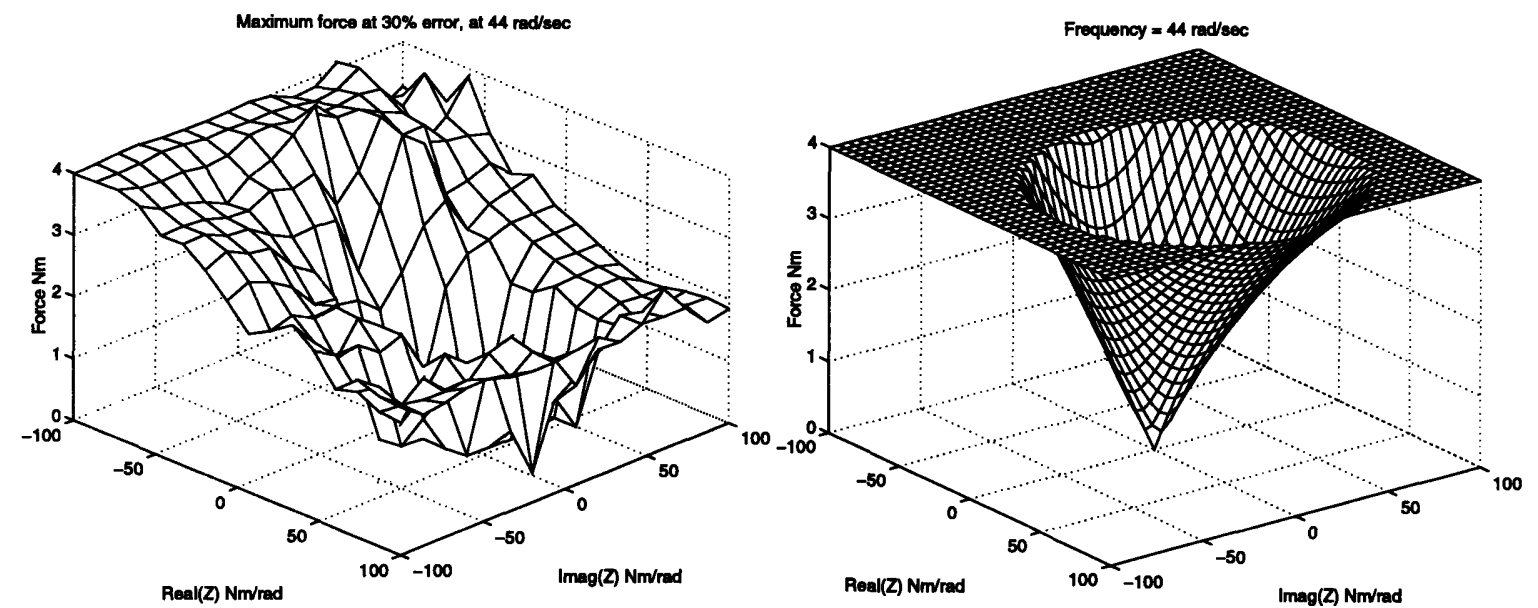

Figure 6-17: Performance results for $44 \mathrm{rad} / \mathrm{sec}$

When the frequency of the test is increased to $7 \mathrm{~Hz}$, or $44 \mathrm{rad} / \mathrm{sec}$, the effect of the spring becomes more clear (Figure 6-17). Generally the actuator can only output forces for large impedances (that is where the position motion is small). However, for 

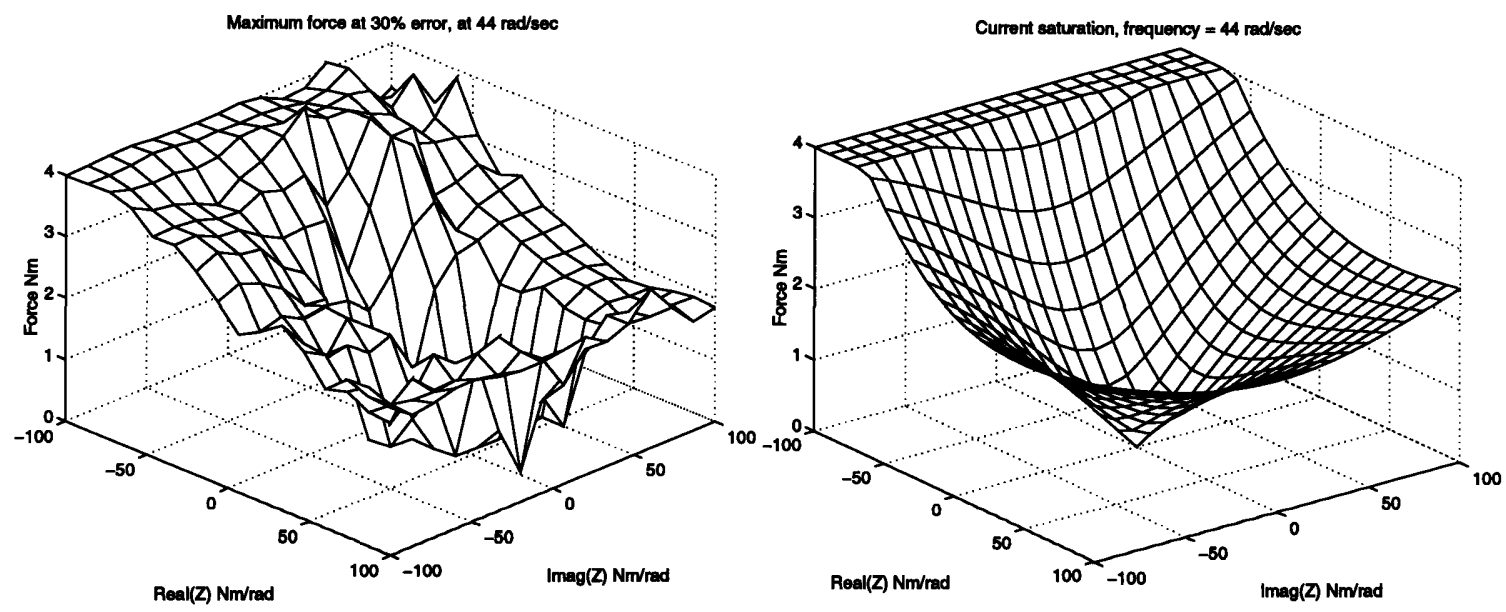

Figure 6-18: Performance results for $44 \mathrm{rad} / \mathrm{sec}$, including efficiency

impedances which have a negative real part-which are ones corresponding to springlike behaviour, the motor can produce forces near its limit. Impedances which are mass like (positive real part) are more difficult for the actuator. This is the general shape predicted by the theory, but the effect is not so pronounced. One reason for this might be that the motor is not completely efficient. A comparison between the data, and the performance of the motor assuming the quoted values for the motor and gearhead efficiency (chapter 5, table 5.1) is included in Figure 6-18. The plot taking into account the efficiency of the motor matches the shape of the data much better.

It is reasonable to expect that the efficiency will change with the operating conditions, as the power losses overcoming friction in the system will change. The friction has two main components, static friction, (or stiction) which occurs when the gears begin to move, and dynamic friction, caused by the motor having to work against the frictional forces of the gearteeth rubbing against each other, and the commutation brushes. The dynamic friction will also have a viscous element, due to the lubrication fluid. The static effects are likely to dominate at low frequencies, and at higher frequencies and forces the dynamic friction will dominate. The dynamic friction will vary with the load on the motor (the frictional force is proportional to the normal force), and the power loss will vary with frequency (power loss will be proportional to the velocity of slipping). Thus the efficiency of the motor is expected to deteriorate for high loads and high speeds.

As the frequency is increased above $7 \mathrm{~Hz}$, the effect of the spring becomes more pronounced. In fact it becomes difficult for the actual motor to produce any force at a mass-like impedance, and it can only produce forces when it is acting like a spring. Figure 6-19 shows a closeup of the spring-like section of the impedance plane for a test at $8 \mathrm{~Hz}$, along with theoretical data taking into account the efficiency of the motor. The rough shape is again similar, and it almost looks as if the motor is behaving 
better than expected in some areas. This is most probably due to a poor estimate of the motor efficiency.
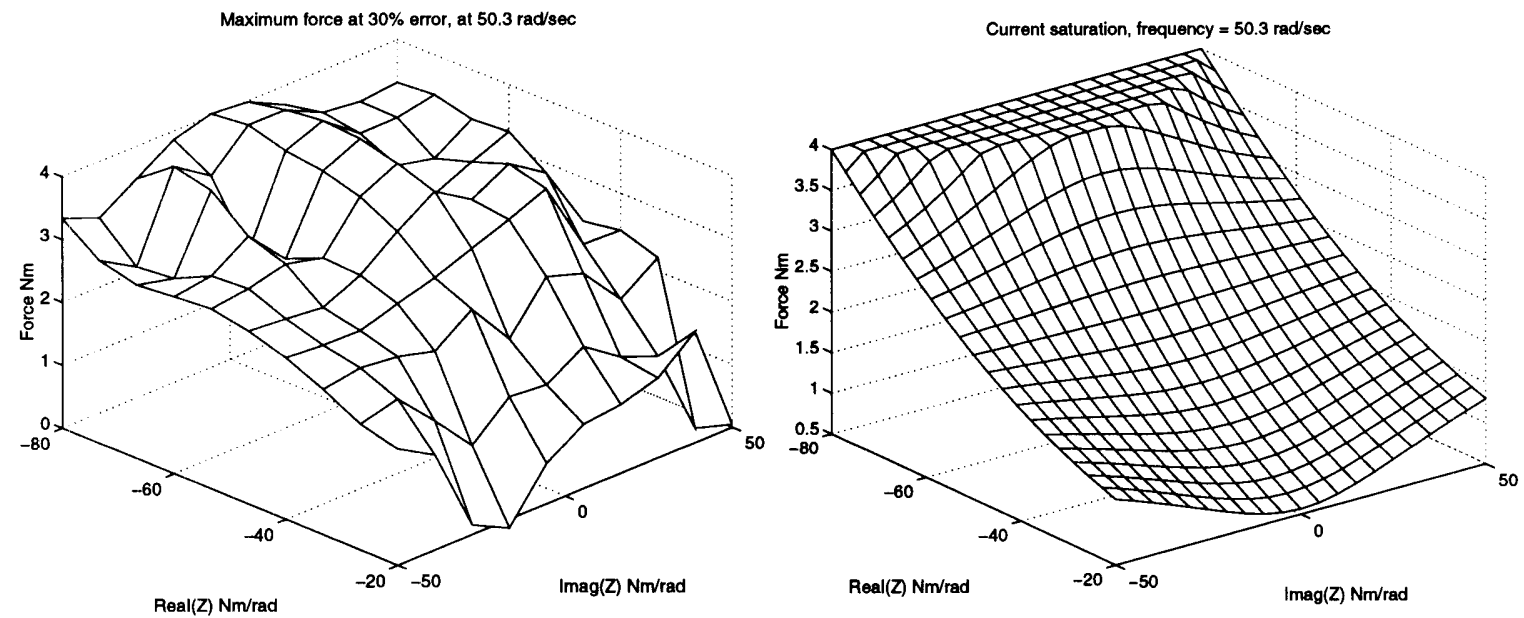

Figure 6-19: Performance results for $50.3 \mathrm{rad} / \mathrm{sec}$

Figure $6-20$ shows a result for $10 \mathrm{~Hz}$. Here the spring characteristic is very pronounced. The size of the peak is smaller too, only about $2.7 \mathrm{Nm}$, as opposed to $4 \mathrm{Nm}$ before. The shape matches the theoretical data reasonably well. The position of the peak corresponds to a negative real impedance of $55 \mathrm{Nm} / \mathrm{rad}$, which compares well stiffness from the control loop bode plots ( $58 \mathrm{Nm} / \mathrm{rad}$, see section 6.4 )
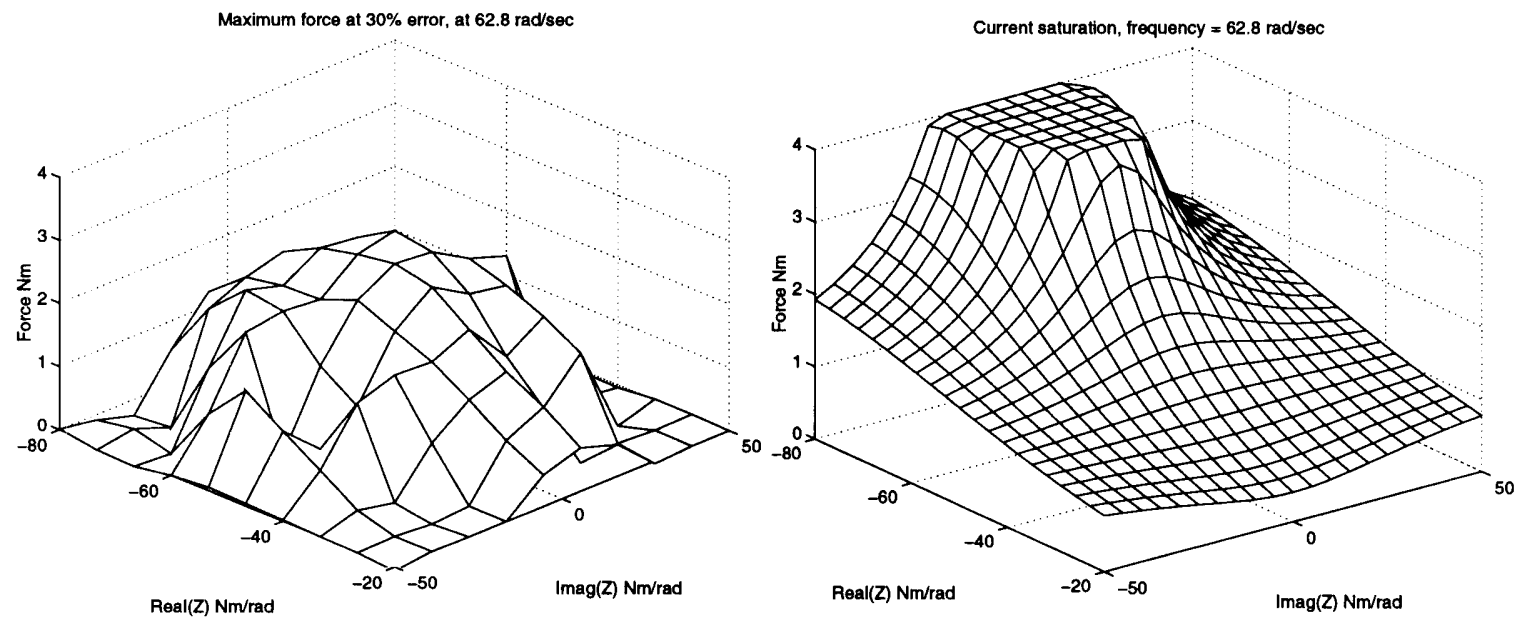

Figure 6-20: Performance results for $62.8 \mathrm{rad} / \mathrm{sec}$

A final run at $15 \mathrm{~Hz}$ is included in Figure 6-21. The actual performance is better than indicated in this plot, the results being affected by limitations in the position controlled motor, which could not provide the position motion required at this speed. A small peak in the plot can be seen, at about $50 \mathrm{Nm} / \mathrm{rad}$, which corresponds well to the measured spring stiffness. 

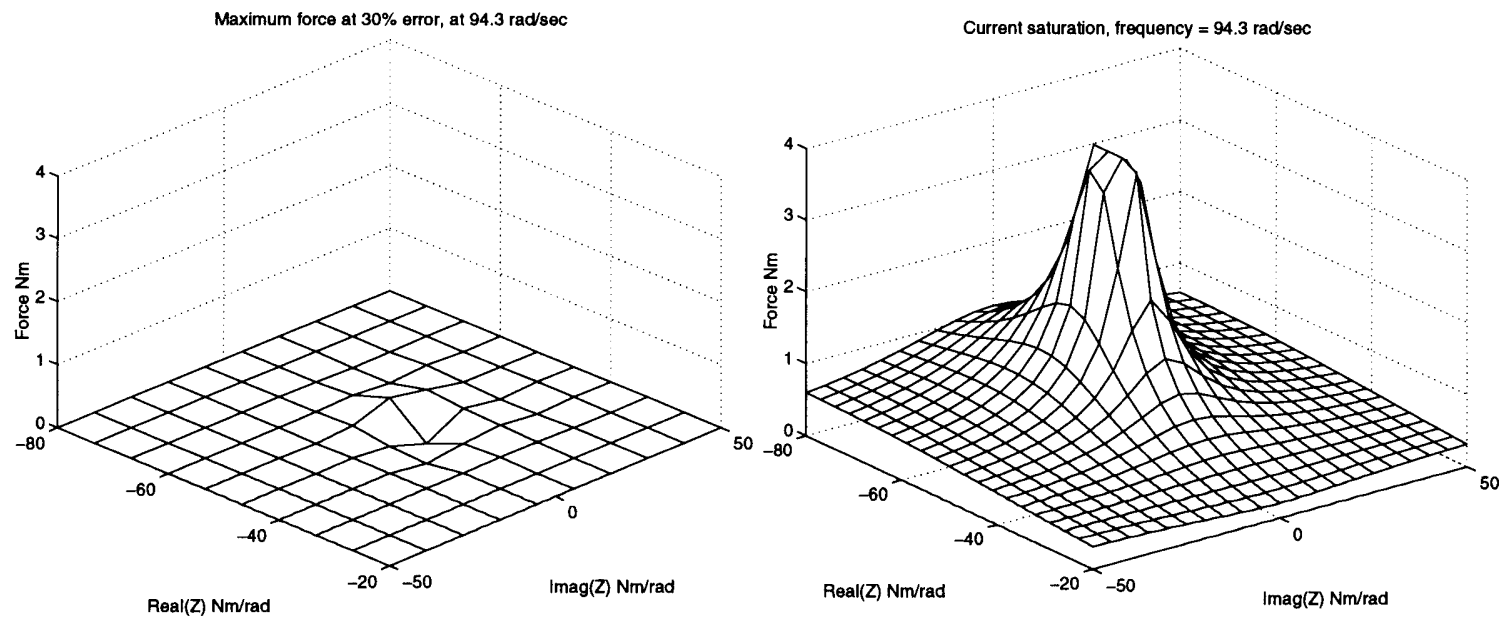

Figure 6-21: Performance results for $94.3 \mathrm{rad} / \mathrm{sec}$

\subsection{Summary}

The performance of the actuator has been determined, using both linear techniques, and impedance/saturation representations. In the linear case the backlash of the motor was found to affect the results. In practice the high frequency commanded sinusoid is unlikely to occur, so the backlash effect may not be significant. Its effect is not noticeable in the step and sine wave responses included in this chapter.

The saturation performance limits were found to have a similar shape to that predicted by the theory, and compare well if the efficiency of the motor is taken into account. The actuator behaves well at low frequencies, and the spring like behaviour is very clear at high frequencies. 


\section{Chapter 7}

\section{Applications}

\section{$7.1 \quad \operatorname{Cog}$}

Series elastic actuators similar to that described in this thesis are being used for the arms of the humanoid robot $\operatorname{Cog}[7]$. The robot will interact with humans and perform human-like tasks, so it is important that its arms are safe. The arms must not be damaged by unexpected collisions, nor must it damage its operator! The actuators are being used to give the arm natural compliance which will aid it in performing tasks. The actuators will also make the arm stable while in contact with all environments. The shock load protection offered by the actuators will also be an advantage, given the unstructured environment in which the arm will operate.

Figure 7-1 shows a photograph of the arm of the robot. It has six degrees of freedom, two each at the shoulder elbow and wrist, all using series elasticity. A hand is being built by another student (Yoky Matsuoka). The use of the spring does introduce complexity into the mechanical design, and for the wrist a cable transmission was used to tuck the motor out of the way of the hand. The arm is described more thoroughly in $[40]$.

\subsection{Planetary rover}

It is also hoped to use the elastic actuators in an arm for a planetary explorer. The arm will be used to collect samples of rocks digging, collecting soil samples and other exploratory tasks. The contact nature of the tasks and the unstructured environment make the choice of series elastic actuators appealing.

The arm for this application is at present in an early design stage, but will probably have only four degrees of freedom, and a simple gripper. 


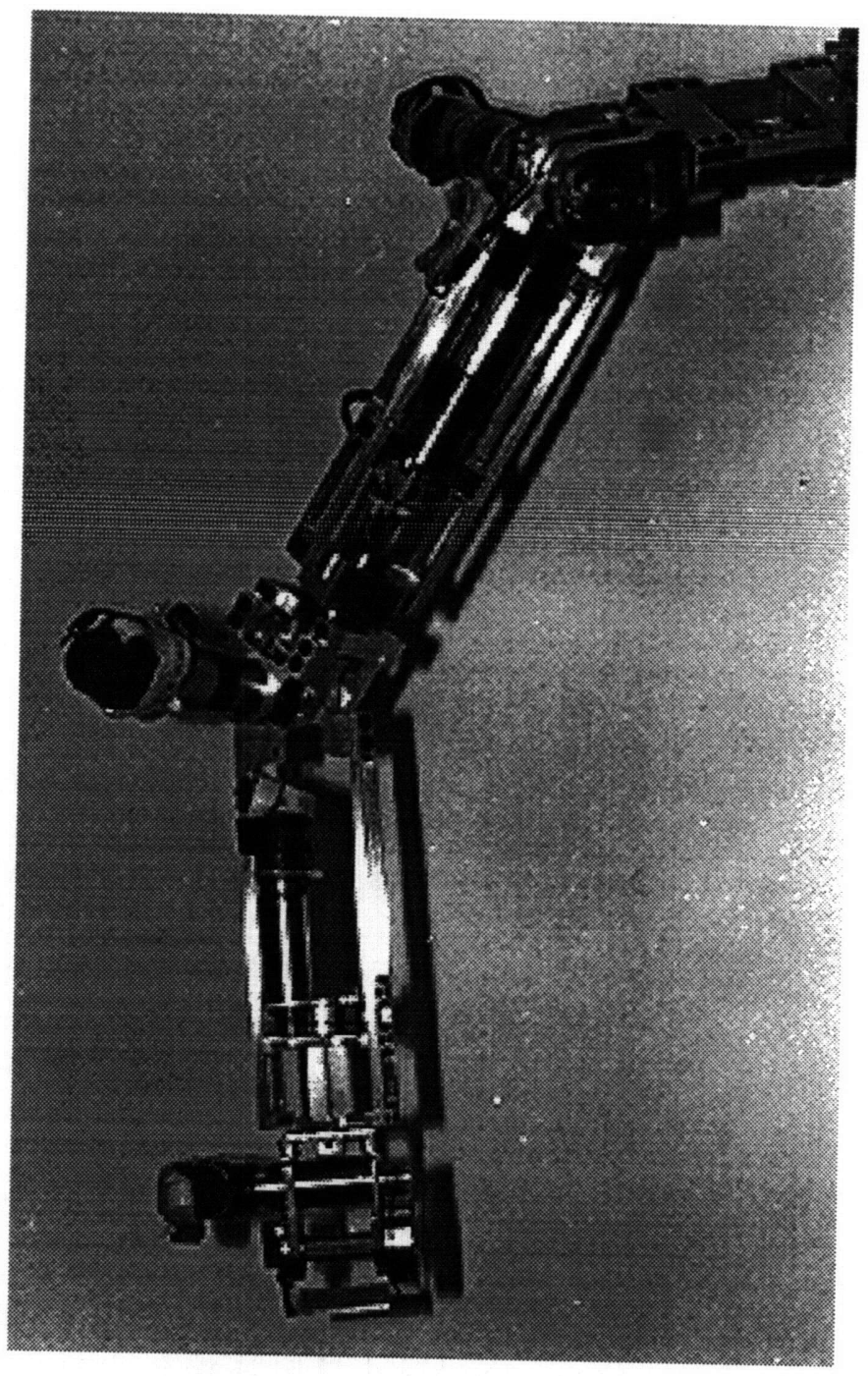

Figure 7-1: Photograph of robot arm

\subsection{Biped Robot}

Another group at MIT is building a biped walking robot using series elastic actuators. The design of the actuators differs in that instead of using a torsional spring, they use tendons with ordinary tension springs to provide the elastic element. The contact with the ground, and the repetitive motion associated with walking make series elastic actuators good choices for this application. 


\section{Chapter 8}

\section{Conclusions}

\subsection{Review of Thesis}

Conventional robotic actuators suffer from a number of problems when it comes to providing good torque control. A way to address this problem has been presented in this thesis. If an elastic element is placed in series with the output of an electric motor, the force control performance of the motor is improved. The motor is isolated from shock loads, and the effects of backlash, torque ripple and friction are filtered by the elastic element.

A further advantage is that the actuator exhibits stable behaviour while in contact with all environments, a quality which has been hard to attain with a conventional electric motor based actuators. Along with the benefits of elasticity come disadvantages which include a limit on the maximum force that the actuator can output due to the mechanical properties of the elastic element, a reduction in the force control bandwidth due to the low pass nature of the spring, and increased complexity and bulk in the mechanical design.

An trial actuator has been built and tested, and some guidelines have been found for the design of the spring, and the selection of the force sensor. Some mathematical theory has been developed for the actuator, not only for its performance limits, but also for its control. These theories have been tested and their deficiencies and strong points noted.

\subsection{Further Work}

The areas of further work fall into three main categories, tying up lose ends of the thesis, improvements of the actuator itself, and further development of the use of series elasticity.

In chapter 6 , the efficiency of the motor was discussed. It would be good to evaluate how the efficiency of the motor system changes with its operating conditions. This would allow the model of the actuator to be improved. A study on the effects 
of backlash on the system performance would also be useful, isolating the causes for the behaviour seen in chapter 6 .

The mechanical design of the spring is another area where further work would be useful, perhaps finding a more refined model of the spring behaviour, or some more design guidelines. This would ease the design of future actuators.

The performance of the actuator could be improved if a gearbox with a lower backlash was chosen. The spring does lessen its effect, but if there was less backlash the system performance would be better. The use of cable transmissions and an alternative type of spring is a possible avenue of research.

The electronic hardware for the actuator is quite esoteric and awkward to work with, however it seems to work pretty well. A proper current controlled motor driver and more resolution in the $\mathrm{A} / \mathrm{D}$ channels would probably increase performance. A controller chip with floating point capability which could be programmed in something other than assembly language would certainly speed up future development.

Further uses for series elasticity have been discussed in part in chapter 7. The main area of work is to integrate a number of actuators into a working robot manipulator, and determine the overall system performance.

Other developments include using two actuators coupled in parallel to achieve higher force control bandwidth. The elasticity isolates each motor output from the load so they can be tuned to have performance at different frequencies. For example, one can be tuned to give high frequency response (using high gear ratios and and low spring constants) and the other high frequency response (low gear ratio and a stiff spring). The overall system will have the performance of the sum of the two actuators. A similar idea is used in loudspeaker design, where the tweeter (high frequency) and the woofer (low frequency) are coupled by air (elasticity) to give performance over a wide frequency range. This idea is currently being investigated at MIT by Salisbury and Morrell [23].

Another interesting idea is to use variable rate springs, where the changing stiffness alters the bandwidth of the force control as the load changes. This is being investigated by Salisbury at MIT. 


\section{Bibliography}

[1] An, H. C., "Trajectory and Force Control of a Direct Drive Arm" PhD Thesis, Massachusetts Institute of Technology, 1986.

[2] An, H. C., Atkeson, C. G., and Hollerbach, J. M., "Model-Based Control of a Robot Manipulator", MIT Press, Cambridge MA., 1988.

[3] Asada, H., and Kanade, T., "Design of Direct-Drive Mechanical Arms", ASME Journal of Vibration, Acoustics, Stress, and Reliability in Design, 105(3), pp. 312-316.

[4] Balas, M. J., "Active Control Control of Flexible Systems", Proc of the 1977 Symposium on Dynamics and Control of Large Flexible Spacecraft, June 13-15, 1977, Blacksburg, VA pp. 217-236.

[5] Benham, P. P., and Crawford, R. J. "Mechanics of Engineering Materials", Longman, New York, NY., 1987.

[6] Brooks, R. A., "The L Manual", IS Robotics, Cambridge MA., 1994.

[7] Brooks, R. A., and Stein, L. A., "Building brains for bodies", to appear in Autonomous Robots, (1:1), 1994.

[8] Cannon, R. H., and Rosenthal, D. E., "Experiments in Control of Flexible Structures with Noncolocated Sensors and Actuators", AIAA Journal of Guidance and Control, Vol. 3, No. 3, pp. 546-553, Sept-Oct., 1984.

[9] Cannon, R. H., and Schmitz, E., "Initial Experiments on the End-Point Control of a Flexible One-Link Robot", Intl J. of Robotics Research, Vol. 3, No. 3, Fall 1984 .

[10] Chen, K-P., and Li-Chen, F., "Nonlinear Adaptive Motion Control for a Manipulator with Flexible Joints", IEEE Intl Conf on Robotics and Automation, 1989, pp. 1201-1206.

[11] Colgate, E., and Hogan, N., "An analysis of Contact Instability in Terms of Passive Equivalents", IEEE Intl Conf on Robotics and Automation, 1989, pp. 404-409. 
[12] Conrad, F., Sørensen, P. H., Trostmann, E., and Zhou, J. J., "On the Mechanical Design and Digital Adaptive Control of the Fast TUD-Hydraulic Test Robot Manipulator", ASME Winter Annual Meeting, Atlanta GA., December 1-6 1991.

[13] Drake, S., "Using Compliance in Lieu of Sensory Feedback for Automatic Assembly", PhD Thesis, Mechanical Engineering Department, Massachusetts Institute of Technology.

[14] Eppinger, S. D., and Seering, W. P., "Three Dynamic Problems in Robot Force Control", IEEE Intl Conf on Robotics and Automation, 1989.

[15] Eppinger, S. D., and Seering, W. P., "Understanding Bandwidth Limitations in Robot Force Control", IEEE Intl Conf on Robotics and Automation, April, 1987.

[16] Hashimoto, M., and Imamura, Y., "An instrumented Compliant Wrist using a Parallel Mechanism", ASME JAPAN/USA Symposium on Flexible Automation, 1992, pp. 741-744.

[17] Hogan, N., "Impedance Control: An approach to manipulation: Part 1 - theory, Part 2 - Implementation, and Part 3 - Applications.", ASME J. of Dynamic Systems, Measurement and Control, 107: 1-24.

[18] Hogan, N., "On the Stability of Manipulators Performing Contact Tasks", IEEE Journal of Robotics and Automation, Vol. 4, No. 6, December 1988.

[19] Hunter, I. W., Hollerbach, J. M., and Ballantyne, J., "A Comparative Analysis of Actuator Technologies for Robotics", Robotic Review 2, MIT Press, 1991.

[20] Khalil, H. K., "Nonlinear Systems", Macmillan, 1992

[21] Lee. M. H., Baek, W. B., Lee, J.N., Yoon, K. S., and Hong, K. S., "A Stabilizing Controller Design for Flexible Joint Robot Manipulators", IEEE Intl Conf on Robotics and Automation, 1993, pp. 805-809.

[22] Marth, G. T., Tarn, T. J., and Bejczy, A. K., "An Event Based Approach to Impact Control: Theory and Experiments", IEEE Intl Conf on Robotics and Automation, 1994, pp. 918-923.

[23] Morrell. J. B., and Salisbury, J. K., "Parallel Coupled Actuator for High Performance Force Control: A Micro-Macro Concept", submitted to IROS 95, Pittsburg.

[24] Raibert, M. H., "Legged Robots that Balance", MIT Press, Cambridge MA, 1986.

[25] Raibert, M. H., and Craig, J. J., "Hybrid Position/Force Control of Manipulators", Trans ASME Journal of Dynamic Systems, Measurement and Control, (103), pp. 126-133, June 1981. 
[26] Roberts, R. K., "The Compliance of End Effector Force Sensors for Robot Manipulator Control", PhD Thesis, Purdue Univ., School of Electrical Engineering.

[27] Society of Automotive Engineers Spring Committee, "Spring Design Manual", 1990

[28] Salisbury, J. K., "Kinematic and force analysis of articulated hands", Robot Hands and the Mechanics of Manipulation, ed Mason, M. T., and Salisbury, J. K., MIT Press, Cambridge, MA., 1985.

[29] Salisbury, J. K., Townsend, W. T., Eberman, B. S., and DiPietro, D. M., "Preliminary Design of a Whole arm Manipulation System (WAMS)", IEEE Intl Conf on Robotics and Automation, 1988.

[30] Salisbury, J. K., "Active Stiffness Control of a Manipulator in Cartesian Coordinates", 19th IEEE Conference on Decision and Control, Dec, 1990, pp. 95-100.

[31] Spong, M. W., "Modelling and Control of Elastic Joint Robots", Trans of the ASME Journal of Dynamic Systems, Measurement and Control, (109), December 1987, pp. 310-319.

[32] Sugano, S., Tsuto, S. and Kato, I., "Force Control of the Robot Finger Joint equipped with Mechanical Compliance Adjuster", Proc of the 1992 IEEE/RSJ Intl. Conf. on Intellignent Robots and Systems, 1992, pp. 2005-2013.

[33] Measurements Group, Micro Measurements Division., Catalogue Part 1, Strain gauge listings.

[34] Townsend, W. T., "The Effect of Transmission Design on Force-Controlled Manipulator Performance", Technical Report AI-TR 900, MIT Artificial Intelligence Laboratory, 1988.

[35] Tuttle, T. D., "Understanding and Modeling the Behavior of a Harmonic Drive Gear Transmission", Technical Report AI-TR 1365, MIT Artificial Intelligence Laboratory, 1992.

[36] Volpi, R. and Khosla, P., "A Theoretical and Experimental Investigation of Explicit Force Control Stategies for Manipulators", IEEE Trans. Automatic Control, Vol. 38, No. 11, November 1993.

[37] Waldron, K. J., Vohnout, V. J., Pery, A., and McGhee, R. B., " Configuration design of the adaptive suspension vehicle", Intl J. Robotics Research, 3:37-48, 1984 .

[38] Whitney, D. E., "Historical Perspective and State of the Art in Robot Force Contol", Intl J. of Robotics Research, Vol 6, No. 1, Spring 1987. 
[39] Whitney, D. E., "Force Feedback Control of Manipulator Fine Motions", Trans ASME Journal of Dynamic Systems, Measurement and Control (99), June 1977, pp. 91-97.

[40] Williamson, M. M., "Design of a naturally compliant arm for a humanoid robot", Submitted to IJCAI 95, Montreal, August 1995.

[41] Wu, C. H,. and Paul R., "Manipulator Compliance based on Joint Torque Control", Proc IEEE Conf on Decision and Control, Alberquerque, NM., pp. 88-94.

[42] Xu, Y., Hollerbach, J. M., and Ma, D., "Force and Transient Control Using Nonlinear PD Control", IEEE Intl Conf on Robotics and Automation, 1994, pp. 924-930.

[43] Xu, Y., Paul, R. P., and Corke, P. I., "Hybrid Position Force Control of Robot Manipulator with an Instrumented Compliant Wrist" Lecture Notes in control and Information Sciences, ed Hayward et al., Springer-Verlag, No.139, pp. 244270.

[44] Yousef-Toumi, K., and Gutz, D. A., "Impact and Force Control", IEEE Intl Conf on Robotics and Automation, 1989, pp. 410-416. 